\title{
Perturbation by Non-Local Operators
}

\author{
Zhen-Qing Chen* and Jie-Ming Wang ${ }^{\dagger}$
}

(September 28, 2016)

\begin{abstract}
Suppose that $d \geq 1$ and $0<\beta<\alpha<2$. We establish the existence and uniqueness of the fundamental solution $q^{b}(t, x, y)$ to a class of (typically nonsymmetric) non-local operators $\mathcal{L}^{b}=\Delta^{\alpha / 2}+\mathcal{S}^{b}$, where

$$
\mathcal{S}^{b} f(x):=\mathcal{A}(d,-\beta) \int_{\mathbb{R}^{d}}\left(f(x+z)-f(x)-\nabla f(x) \cdot z \mathbb{1}_{\{|z| \leq 1\}}\right) \frac{b(x, z)}{|z|^{d+\beta}} d z
$$

and $b(x, z)$ is a bounded measurable function on $\mathbb{R}^{d} \times \mathbb{R}^{d}$ with $b(x, z)=b(x,-z)$ for $x, z \in \mathbb{R}^{d}$. Here $\mathcal{A}(d,-\beta)$ is a normalizing constant so that $\mathcal{S}^{b}=\Delta^{\beta / 2}$ when $b(x, z) \equiv 1$. We show that if $b(x, z) \geq-\frac{\mathcal{A}(d,-\alpha)}{\mathcal{A}(d,-\beta)}|z|^{\beta-\alpha}$, then $q^{b}(t, x, y)$ is a strictly positive continuous function and it uniquely determines a conservative Feller process $X^{b}$, which has strong Feller property. The Feller process $X^{b}$ is the unique solution to the martingale problem of $\left(\mathcal{L}^{b}, \mathcal{S}\left(\mathbb{R}^{d}\right)\right)$, where $\mathcal{S}\left(\mathbb{R}^{d}\right)$ denotes the space of tempered functions on $\mathbb{R}^{d}$. Furthermore, sharp two-sided estimates on $q^{b}(t, x, y)$ are derived. In stark contrast with the gradient perturbations, these estimates exhibit different behaviors for different types of $b(x, z)$. The model considered in this paper contains the following as a special case. Let $Y$ and $Z$ be (rotationally) symmetric $\alpha$-stable process and symmetric $\beta$-stable processes on $\mathbb{R}^{d}$, respectively, that are independent to each other. Solution to stochastic differential equations $d X_{t}=d Y_{t}+c\left(X_{t-}\right) d Z_{t}$ has infinitesimal generator $\mathcal{L}^{b}$ with $b(x, z)=|c(x)|^{\beta}$.
\end{abstract}

AMS 2010 Mathematics Subject Classification: Primary 60J35, 47G20, 60J75; Secondary 47D07

Keywords and phrases: symmetric stable process, fractional Laplacian, perturbation, nonlocal operator, integral kernel, positivity, Lévy system, Feller semigroup, martingale problem

\section{Introduction}

Let $d \geq 1$ be an integer and $0<\beta<\alpha<2$. For integer $k \geq 1$, denote by $C_{b}^{k}\left(\mathbb{R}^{d}\right)\left(\right.$ resp. $\left.C_{c}^{k}\left(\mathbb{R}^{d}\right)\right)$ the space of continuous functions on $\mathbb{R}^{d}$ that have bounded continuous partial derivatives up to order $k$ (resp. the space of continuous functions on $\mathbb{R}^{d}$ with compact support that have continuous partial derivatives up to order $k)$. Recall that a stochastic process $Y=\left(Y_{t}, \mathbb{P}_{x}, x \in\right.$ $\mathbb{R}^{d}$ ) on $\mathbb{R}^{d}$ is called a (rotationally) symmetric $\alpha$-stable process on $\mathbb{R}^{d}$ if it is a Lévy process having

$$
\mathbb{E}_{x}\left[e^{i \xi \cdot\left(Y_{t}-Y_{0}\right)}\right]=e^{-t|\xi|^{\alpha}} \quad \text { for every } x, \xi \in \mathbb{R}^{d}
$$

* Research partially supported by NSF Grant DMS-1206276, and NNSFC Grant 11128101.

${ }^{\dagger}$ Corresponding author. Research partially supported by NNSFC Grant 11401025. 
Let $\widehat{f}(\xi):=\int_{\mathbb{R}^{d}} e^{i \xi \cdot x} f(x) d x$ denote the Fourier transform of a function $f$ on $\mathbb{R}^{d}$. The fractional Laplacian $\Delta^{\alpha / 2}$ on $\mathbb{R}^{d}$ is defined as

$$
\Delta^{\alpha / 2} f(x)=\int_{\mathbb{R}^{d}}\left(f(x+z)-f(x)-\nabla f(x) \cdot z \mathbb{1}_{\{|z| \leq 1\}}\right) \frac{\mathcal{A}(d,-\alpha)}{|z|^{d+\alpha}} d z
$$

for $f \in C_{b}^{2}\left(\mathbb{R}^{d}\right)$. Here $\mathcal{A}(d,-\alpha)=\Gamma((d+\alpha) / 2) /\left(2^{-\alpha} \pi^{d / 2}|\Gamma(-\alpha / 2)|\right)$, which is the normalizing constant so that $\widehat{\Delta^{\alpha / 2} f}(\xi)=-|\xi|^{\alpha} \widehat{f}(\xi)$. Hence $\Delta^{\alpha / 2}$ is the infinitesimal generator for the symmetric $\alpha$-stable process on $\mathbb{R}^{d}$.

Throughout this paper, $b(x, z)$ is a real-valued bounded function on $\mathbb{R}^{d} \times \mathbb{R}^{d}$ satisfying

$$
b(x, z)=b(x,-z) \quad \text { for every } x, z \in \mathbb{R}^{d} .
$$

This paper is concerned with the existence, uniqueness and sharp two-sided estimates on the "fundamental solution" of the following non-local operator on $\mathbb{R}^{d}$,

$$
\mathcal{L}^{b} f(x)=\Delta^{\alpha / 2} f(x)+\mathcal{S}^{b} f(x), \quad f \in C_{b}^{2}\left(\mathbb{R}^{d}\right),
$$

where

$$
\mathcal{S}^{b} f(x):=\mathcal{A}(d,-\beta) \int_{\mathbb{R}^{d}}\left(f(x+z)-f(x)-\nabla f(x) \cdot z \mathbb{1}_{\{|z| \leq 1\}}\right) \frac{b(x, z)}{|z|^{d+\beta}} d z .
$$

We point out that since $b(x, z)$ satisfies condition (1.2), the truncation $|z| \leq 1$ in (1.3) can be replaced by $|z| \leq \lambda$ for any $\lambda>0$; that is, for every $\lambda>0$,

$$
\mathcal{S}^{b} f(x)=\mathcal{A}(d,-\beta) \int_{\mathbb{R}^{d}}\left(f(x+z)-f(x)-\langle\nabla f(x), z\rangle \mathbb{1}_{\{|z| \leq \lambda\}}\right) \frac{b(x, z)}{|z|^{d+\beta}} d z .
$$

In fact, under condition (1.2),

$$
\begin{aligned}
\mathcal{S}^{b} f(x) & =\mathcal{A}(d,-\beta) \text { p.v. } \int_{\mathbb{R}^{d}}(f(x+z)-f(x)) \frac{b(x, z)}{|z|^{d+\beta}} d z \\
& :=\mathcal{A}(d,-\beta) \lim _{\varepsilon \rightarrow 0} \int_{\left\{z \in \mathbb{R}^{d}:|z|>\varepsilon\right\}}(f(x+z)-f(x)) \frac{b(x, z)}{|z|^{d+\beta}} d z .
\end{aligned}
$$

Condition (1.2) allows us to reduce general bounded measurable function $b$ on $\mathbb{R}^{d} \times \mathbb{R}^{d}$ to the situation where $\|b\|_{\infty}$ is sufficiently small through a scaling argument (see (3.18) and Lemma 3.5). The operator $\mathcal{L}^{b}$ is in general non-symmetric. Clearly, $\mathcal{L}^{b}=\Delta^{\alpha / 2}$ when $b \equiv 0$ and $\mathcal{L}^{b}=\Delta^{\alpha / 2}+\Delta^{\beta / 2}$ when $b \equiv 1$.

We are led to the study of this non-local operator $\mathcal{L}^{b}$ by the consideration of the following stochastic differential equation $(\mathrm{SDE})$ on $\mathbb{R}^{d}$ :

$$
d X_{t}=d Y_{t}+c\left(X_{t-}\right) d Z_{t}
$$

where $Y$ is a symmetric $\alpha$-stable process on $\mathbb{R}^{d}$ and $Z$ is an independent symmetric $\beta$-stable process with $0<\beta<\alpha$. Such SDE arises naturally in applications when there are more than one sources of random noises. When $c$ is a bounded Lipschitz function on $\mathbb{R}^{d}$, it is easy to show using Picard's iteration method that for every $x \in \mathbb{R}^{d}$, SDE (1.6) has a unique strong solution with $X_{0}=x$. We denote the law of such a solution by $\mathbb{P}_{x}$. The collection of the solutions $\left(X_{t}, \mathbb{P}_{x}, x \in \mathbb{R}^{d}\right)$ forms a strong Markov process $X$ on $\mathbb{R}^{d}$. Using Ito's formula, one concludes 
that the infinitesimal generator of $X$ is $\mathcal{L}^{b}$ with $b(x, z)=|c(x)|^{\beta}$ and so in this case $X$ solves the martingale problem for $\left(\mathcal{L}^{b}, C_{b}^{2}\left(\mathbb{R}^{d}\right)\right)$. The following questions arise naturally: does the Markov process $X$ have a transition density function? If so, what is its sharp two-sided estimates? Is there a solution to the martingale problem for $\Delta^{\alpha / 2}+|c(x)|^{\beta} \Delta^{\beta / 2}$ when $c$ is not Lipschitz continuous? We will address these questions for the more general operator $\mathcal{L}^{b}$ in this paper.

Heat kernel analysis is an important subject in analysis and in probability theory, as heat kernel encodes all the information about the corresponding infinitesimal generator and the corresponding Markov processes. Since explicit formula can only be derived in some very special and limited cases, the main focus of the heat kernel analysis is on its sharp estimates. While it is relatively easy to get some crude bounds, obtaining sharp two-sided bounds on the heat kernel is typically quite challenging. It requires deep understanding of the corresponding generator. Heat kernel estimates for discontinuous Markov processes have been under intense study recently. Most results obtained so far are mainly for symmetric Markov processes. See [7] for a recent survey. It is well known that the study of non-symmetric operators requires different approaches and techniques than that for symmetric operators. Results of this paper can also be viewed as an attempt in establishing heat kernel estimates for non-symmetric discontinuous Markov processes. For example, Corollary 1.4 and Theorem 1.5 can be viewed as the non-symmetric analogy, though in a restricted setting, of the two-sided heat kernel estimates for symmetric stable-like processes and mixed stable-like processes established in [11] and [12], respectively. See Remark 1.7 below for more information on heat kernel analysis.

For $a \geq 0$, denote by $p_{a}(t, x, y)$ the fundamental function of $\Delta^{\alpha / 2}+a \Delta^{\beta / 2}$ (or equivalently, the transition density function of the Lévy process $\left.Y_{t}+a^{1 / \beta} Z_{t}\right)$. Clearly, $p_{a}(t, x, y)$ is a function of $t$ and $x-y$, so sometimes we also write it as $p_{a}(t, x-y)$. It is known (see (2.3) of Section 2 for details) that on $(0, \infty) \times \mathbb{R}^{d} \times \mathbb{R}^{d}$,

$$
\begin{aligned}
& p_{0}(t, x, y) \asymp t^{-d / \alpha} \wedge \frac{t}{|x-y|^{d+\alpha}}, \\
& p_{a}(t, x, y) \asymp\left(t^{-d / \alpha} \wedge(a t)^{-d / \beta}\right) \wedge\left(\frac{t}{|x-y|^{d+\alpha}}+\frac{a t}{|x-y|^{d+\beta}}\right) .
\end{aligned}
$$

Here for two non-negative functions $f$ and $g$, the notation $f \asymp g$ means that there is a constant $c \geq 1$ so that $c^{-1} f \leq g \leq c f$ on their common domain of definitions. For real numbers $a, c \in \mathbb{R}$, we use $a \vee c$ and $a \wedge c$ to denote $\max \{a, c\}$ and $\min \{a, c\}$, respectively. We point out that the comparison constants in (1.8) is independent of $a>0$; see (2.3) in Section 2. Using the observation that $a \wedge b \asymp \frac{a b}{a+b}$, one concludes from (1.7) that

$$
p_{0}(t, x, y) \asymp \frac{t}{\left(t^{1 / \alpha}+|x-y|\right)^{d+\alpha}} \quad \text { on }(0, \infty) \times \mathbb{R}^{d} \times \mathbb{R}^{d} .
$$

Note that $(a t)^{-d / \beta} \geq t^{-d / \alpha}$ whenever $0<t \leq a^{-\alpha /(\alpha-\beta)}$. Thus for every $k>0$,

$$
p_{a}(t, x, y) \asymp t^{-d / \alpha} \wedge\left(\frac{t}{|x-y|^{d+\alpha}}+\frac{a t}{|x-y|^{d+\beta}}\right) \quad \text { on }\left(0, k a^{-\alpha /(\alpha-\beta)}\right] \times \mathbb{R}^{d} \times \mathbb{R}^{d},
$$

with the comparison constants depending only on $d, \alpha, \beta$ and $k$.

Since $\mathcal{L}^{b}=\Delta^{\alpha / 2}+\mathcal{S}^{b}$ is a lower order perturbation of $\Delta^{\alpha / 2}$ by $\mathcal{S}^{b}$, heuristically the fundamental solution (or kernel) $q^{b}(t, x, y)$ of $\mathcal{L}^{b}$ should satisfy the following Duhamel's formula:

$$
q^{b}(t, x, y)=p_{0}(t, x, y)+\int_{0}^{t} \int_{\mathbb{R}^{d}} q^{b}(t-s, x, z) \mathcal{S}_{z}^{b} p_{0}(s, z, y) d z d s
$$


for $t>0$ and $x, y \in \mathbb{R}^{d}$. Here the notation $S_{z}^{b} p_{0}(s, z, y)$ means the non-local operator $\mathcal{S}^{b}$ is applied to the function $z \mapsto p_{0}(s, z, y)$. Similar notation will also be used for other operators, for example, $\Delta_{z}^{\alpha / 2}$. Applying (1.11) recursively, it is reasonable to conjecture that $\sum_{n=0}^{\infty} q_{n}^{b}(t, x, y)$, if convergent, is a solution to (1.11), where $q_{0}^{b}(t, x, y):=p_{0}(t, x, y)$ and

$$
q_{n}^{b}(t, x, y):=\int_{0}^{t} \int_{\mathbb{R}^{d}} q_{n-1}^{b}(t-s, x, z) \mathcal{S}_{z}^{b} p_{0}(s, z, y) d z d s \quad \text { for } n \geq 1 .
$$

For each bounded function $b(x, z)$ on $\mathbb{R}^{d} \times \mathbb{R}^{d}$ and $\lambda>0$, define

$$
m_{b, \lambda}=\operatorname{essinf}_{x, z \in \mathbb{R}^{d},|z|>\lambda} b(x, z) \quad \text { and } \quad M_{b, \lambda}=\operatorname{esssup}_{x, z \in \mathbb{R}^{d},|z|>\lambda}|b(x, z)| .
$$

The followings are the main results of this paper.

Theorem 1.1. For every bounded function $b$ on $\mathbb{R}^{d} \times \mathbb{R}^{d}$ satisfying condition (1.2), there is a unique continuous function $q^{b}(t, x, y)$ on $(0, \infty) \times \mathbb{R}^{d} \times \mathbb{R}^{d}$ that satisfies (1.11) on $(0, \varepsilon] \times \mathbb{R}^{d} \times \mathbb{R}^{d}$ with $\left|q^{b}(t, x, y)\right| \leq c p_{1}(t, x, y)$ on $(0, \varepsilon] \times \mathbb{R}^{d} \times \mathbb{R}^{d}$ for some $\varepsilon, c>0$, and that

$$
\int_{\mathbb{R}^{d}} q^{b}(t, x, y) q^{b}(s, y, z) d y=q^{b}(t+s, x, z) \quad \text { for every } t, s>0 \text { and } x, z \in \mathbb{R}^{d} .
$$

Moreover, the following holds.

(i) There is a constant $A_{0}=A_{0}(d, \alpha, \beta)>0$ so that $q^{b}(t, x, y)=\sum_{n=0}^{\infty} q_{n}^{b}(t, x, y)$ on $\left(0,\left(A_{0} /\|b\|_{\infty}\right)^{\alpha /(\alpha-\beta)}\right] \times \mathbb{R}^{d} \times \mathbb{R}^{d}$, where $q_{n}^{b}(t, x, y)$ is defined by (1.12) .

(ii) $q^{b}(t, x, y)$ satisfies the Duhamel's formula (1.11) for all $t>0$ and $x, y \in \mathbb{R}^{d}$. Moreover, $\mathcal{S}_{x}^{b} q^{b}(t, x, y)$ exists pointwise in the sense of (1.5) and

$$
q^{b}(t, x, y)=p_{0}(t, x, y)+\int_{0}^{t} \int_{\mathbb{R}^{d}} p_{0}(t-s, x, z) \mathcal{S}_{z}^{b} q^{b}(s, z, y) d z d s
$$

for $t>0$ and $x, y \in \mathbb{R}^{d}$.

(iii) For each $t>0$ and $x \in \mathbb{R}^{d}, \int_{\mathbb{R}^{d}} q^{b}(t, x, y) d y=1$.

(iv) For every $f \in C_{b}^{2}\left(\mathbb{R}^{d}\right)$,

$$
T_{t}^{b} f(x)-f(x)=\int_{0}^{t} T_{s}^{b} \mathcal{L}^{b} f(x) d s,
$$

where $T_{t}^{b} f(x)=\int_{\mathbb{R}^{d}} q^{b}(t, x, y) f(y) d y$.

(v) Let $A>0$ and $\lambda>0$. There is a positive constant $C=C(d, \alpha, \beta, A, \lambda) \geq 1$ so that for any $b$ satisfying (1.2) with $\|b\|_{\infty} \leq A$,

$$
\left|q^{b}(t, x, y)\right| \leq C e^{C t} p_{M_{b, \lambda}}(t, x, y) \quad \text { on }(0, \infty) \times \mathbb{R}^{d} \times \mathbb{R}^{d} .
$$

We remark that estimate (1.16) allows one to get sharper bound on $\left|q^{b}(t, x, y)\right|$ by selecting optimal $\lambda>0$. When $Z_{t}$ is the deterministic process $t$ and $c$ is an $\mathbb{R}^{d}$-valued bounded Lipschitz function on $\mathbb{R}^{d}$, the solution of (1.6) is a symmetric $\alpha$-stable process with drift. Its infinitesimal generator is $\Delta^{\alpha / 2}+c(x) \nabla$. Existence of integral kernel to $\Delta^{\alpha / 2}+c(x) \nabla$ and its estimates have been studied recently in [6] (in fact, $c$ there can be an $\mathbb{R}^{d}$-valued function in certain Kato class). 
Unlike the gradient perturbation for $\Delta^{\alpha / 2}$, in general the kernel $q^{b}(t, x, y)$ in Theorem 1.1 can take negative values. For example, this is the case when $b \equiv-1$, that is, when $\mathcal{L}^{b}=\Delta^{\alpha / 2}-\Delta^{\beta / 2}$, according to the next theorem. Observe that

$$
\mathcal{L}^{b} f(x)=\int_{\mathbb{R}^{d}}\left(f(x+z)-f(x)-\langle\nabla f(x), z\rangle \mathbb{1}_{\{|z| \leq 1\}}\right) j^{b}(x, z) d z,
$$

where

$$
j^{b}(x, z)=\frac{\mathcal{A}(d,-\alpha)}{|z|^{d+\alpha}}\left(1+\frac{\mathcal{A}(d,-\beta)}{\mathcal{A}(d,-\alpha)} b(x, z)|z|^{\alpha-\beta}\right) .
$$

The next result gives a necessary and sufficient condition for the kernel $q^{b}(t, x, y)$ in Theorem 1.1 to be non-negative when $b(x, z)$ is continuous in $x$ for a.e. $z$.

Theorem 1.2. Let $b$ be a bounded function on $\mathbb{R}^{d} \times \mathbb{R}^{d}$ that satisfies (1.2) and that

$$
x \mapsto b(x, z) \text { is continuous for a.e. } z \in \mathbb{R}^{d} .
$$

Then $q^{b}(t, x, y) \geq 0$ on $(0, \infty) \times \mathbb{R}^{d} \times \mathbb{R}^{d}$ if and only if for each $x \in \mathbb{R}^{d}, j^{b}(x, z) \geq 0$ for a.e. $z \in \mathbb{R}^{d}$; that is, if and only if

$$
b(x, z) \geq-\frac{\mathcal{A}(d,-\alpha)}{\mathcal{A}(d,-\beta)}|z|^{\beta-\alpha} \quad \text { for a.e. } z \in \mathbb{R}^{d} .
$$

In particular, if $b(x, z)=b(x)$ is a function of $x$ only, then $q^{b}(t, x, y) \geq 0$ on $(0, \infty) \times \mathbb{R}^{d} \times \mathbb{R}^{d}$ if and only if $b(x) \geq 0$ on $\mathbb{R}^{d}$.

Next theorem drops the assumption (1.18), gives lower bound estimates and refines upper bound estimates on $q^{b}(t, x, y)$ for $b(x, z)$ satisfying condition (1.19) and makes connections to the martingale problem for $\mathcal{L}^{b}$. To state it, we need first to recall some definitions.

Let $\mathbb{D}\left([0, \infty), \mathbb{R}^{d}\right)$ be the space of right continuous $\mathbb{R}^{d}$-valued functions having left limits on $[0, \infty)$, equipped with Skorokhod topology. Denote by $X_{t}$ the projection coordinate map on $\mathbb{D}\left([0, \infty), \mathbb{R}^{d}\right)$. Let $\mathcal{C}$ be a subspace of $C_{b}^{2}\left(\mathbb{R}^{d}\right)$. A probability measure $Q$ on the Skorokhod space $\mathbb{D}\left([0, \infty), \mathbb{R}^{d}\right)$ is said to to be a solution to the martingale problem for $\left(\mathcal{L}^{b}, \mathcal{C}\right)$ with initial value $x \in \mathbb{R}^{d}$ if $Q\left(X_{0}=x\right)=1$ and for every $f \in \mathcal{C}$,

$$
M_{t}^{f}:=f\left(X_{t}\right)-f\left(X_{0}\right)-\int_{0}^{t} \mathcal{L}^{b} f\left(X_{s}\right) d s
$$

is a $Q$-martingale. The martingale problem $\left(\mathcal{L}^{b}, \mathcal{C}\right)$ with initial value $x \in \mathbb{R}^{d}$ is said to be well-posed if it has a unique solution.

Let $C_{\infty}\left(\mathbb{R}^{d}\right)$ be the space of continuous functions on $\mathbb{R}^{d}$ that vanish at infinity, equipped with supremum norm. Set

$$
C_{\infty}^{2}\left(\mathbb{R}^{d}\right)=\left\{f \in C_{\infty}\left(\mathbb{R}^{d}\right): \text { the first and second derivatives of } f \text { are all in } C_{\infty}\left(\mathbb{R}^{d}\right)\right\} .
$$

A Markov process on $\mathbb{R}^{d}$ is called a Feller process if its transition semigroup is a strongly continuous semigroup in $C_{\infty}\left(\mathbb{R}^{d}\right)$. Feller processes is a class of nice strong Markov processes, 
called Hunt processes (see [15]). Let $\bar{p}_{0}(t, x, y)$ be the fundamental solution of the truncated operator

$$
\bar{\Delta}^{\alpha / 2} f(x)=\int_{|z| \leq 1}\left(f(x+z)-f(x)-\nabla f(x) \cdot z \mathbb{1}_{\{|z| \leq 1\}}\right) \frac{\mathcal{A}(d,-\alpha)}{|z|^{d+\alpha}} d z ;
$$

or, equivalently, $\bar{p}_{0}(t, x, y)$ is the transition density function for the finite range $\alpha$-stable (Lévy) process with Lévy measure $\mathcal{A}(d,-\alpha)|z|^{-(d+\alpha)} \mathbb{1}_{\{|z| \leq 1\}}$. It is established in [8] that $\bar{p}_{0}(t, x, y)$ is jointly continuous and enjoys the following two sided estimates:

$$
\bar{p}_{0}(t, x, y) \asymp t^{-d / \alpha} \wedge \frac{t}{|x-y|^{d+\alpha}}
$$

for $t \in(0,1]$ and $|x-y| \leq 1$, and there are constants $c_{k}=c_{k}(d, \alpha)>0, k=1,2,3,4$ so that

$$
c_{1}\left(\frac{t}{|x-y|}\right)^{c_{2}|x-y|} \leq \bar{p}_{0}(t, x, y) \leq c_{3}\left(\frac{t}{|x-y|}\right)^{c_{4}|x-y|}
$$

for $t \in(0,1]$ and $|x-y|>1$.

Define $b^{+}(x, z)=\max \{b(x, z), 0\}$.

Theorem 1.3. For every $A>0$ and $\lambda>0$, there are positive constants $C_{k}=C_{k}(d, \alpha, \beta, A)$, $k=1,2$, and $C_{3}=C_{3}(d, \alpha, \beta, A, \lambda)$ such that for any bounded $b$ satisfying (1.2) and (1.19) with $\|b\|_{\infty} \leq A$

$$
C_{1} \bar{p}_{0}\left(t, C_{2} x, C_{2} y\right) \leq q^{b}(t, x, y) \leq C_{3} p_{M_{b^{+}, \lambda}}(t, x, y) \quad \text { for } t \in(0,1] \text { and } x, y \in \mathbb{R}^{d} .
$$

Moreover, for every $\varepsilon>0$, there is a positive constant $C_{4}=C_{4}(d, \alpha, \beta, A, \lambda, \varepsilon)$ such that for any $b$ on $\mathbb{R}^{d} \times \mathbb{R}^{d}$ satisfying (1.2) with $\|b\|_{\infty} \leq A$ so that

$$
j^{b}(x, z) \geq \varepsilon|z|^{-(d+\alpha)} \quad \text { for a.e. } x, z \in \mathbb{R}^{d}
$$

we have

$$
C_{4} p_{m_{b^{+}, \lambda}}(t, x, y) \leq q^{b}(t, x, y) \leq C_{3} p_{M_{b^{+}, \lambda}}(t, x, y) \quad \text { for } t \in(0,1] \text { and } x, y \in \mathbb{R}^{d} .
$$

The kernel $q^{b}(t, x, y)$ uniquely determines a Feller process $X^{b}=\left(X_{t}^{b}, t \geq 0, \mathbb{P}_{x}, x \in \mathbb{R}^{d}\right)$ on the canonical Skorokhod space $\mathbb{D}\left([0, \infty), \mathbb{R}^{d}\right)$ such that

$$
\mathbb{E}_{x}\left[f\left(X_{t}^{b}\right)\right]=\int_{\mathbb{R}^{d}} q^{b}(t, x, y) f(y) d y
$$

for every bounded continuous function $f$ on $\mathbb{R}^{d}$. The Feller process $X^{b}$ is conservative and has a Lévy system $\left(J^{b}(x, y) d y, t\right)$, where $J^{b}(x, y)=j^{b}(x, y-x)$.

$$
J^{b}(x, y)=j^{b}(x, y-x)=\frac{\mathcal{A}(d,-\alpha)}{|x-y|^{d+\alpha}}+\frac{\mathcal{A}(d,-\beta) b(x, y-x)}{|x-y|^{d+\beta}} .
$$

Moreover, for each $x \in \mathbb{R}^{d},\left(X^{b}, \mathbb{P}_{x}\right)$ is the unique solution to the martingale problem $\left(\mathcal{L}^{b}, \mathcal{S}\left(\mathbb{R}^{d}\right)\right)$ with initial value $x$. Here $\mathcal{S}\left(\mathbb{R}^{d}\right)$ denotes the space of tempered functions on $\mathbb{R}^{d}$. 
Here we say $\left(J^{b}(x, y) d y, t\right)$ is a Lévy system for $X^{b}$ if for any non-negative measurable function $f$ on $\mathbb{R}_{+} \times \mathbb{R}^{d} \times \mathbb{R}^{d}$ with $f(s, y, y)=0$ for all $y \in \mathbb{R}^{d}$, any stopping time $T$ (with respect to the filtration of $X^{b}$ ) and any $x \in \mathbb{R}^{d}$,

$$
\mathbb{E}_{x}\left[\sum_{s \leq T} f\left(s, X_{s-}^{b}, X_{s}^{b}\right)\right]=\mathbb{E}_{x}\left[\int_{0}^{T}\left(\int_{\mathbb{R}^{d}} f\left(s, X_{s}^{b}, y\right) J^{b}\left(X_{s}^{b}, y\right) d y\right) d s\right] .
$$

A Lévy system for $X^{b}$ describes the jumps of the process $X^{b}$. A Markov process on $\mathbb{R}^{d}$ is said to have strong Feller property if its transition semigroup maps bounded measurable functions on $\mathbb{R}^{d}$ into bounded continuous functions on $\mathbb{R}^{d}$. Since $q^{b}(t, x, y)$ is a continuous function, one has by Theorem 1.1 and the dominated convergence theorem that the Feller process $X^{b}$ of Theorem 1.3 has strong Feller property.

Condition (1.23) is always satisfied if $b(x, z)$ is nonnegative. We emphasize the $m_{b^{+}, \lambda}$ and $M_{b^{+}, \lambda}$ terms appeared in the estimates in Theorem 1.3. Under condition (1.23) and the assumption that $\|b\|_{\infty} \leq A$, the value of $b(x, z)$ on $\mathbb{R}^{d} \times\left\{z \in \mathbb{R}^{d}:|z| \leq \lambda\right\}$ is irrelevant in the estimates of $q^{b}(t, x, y)$ in (1.24). By selecting suitable $\lambda>0$ in (1.24), one can get optimal two-sided estimates on $q^{b}(t, x, y)$. The following follows immediately from Theorem 1.3 by taking a suitable $\lambda>0$.

Corollary 1.4. Let $A \geq 0$ and $\varepsilon>0$. There is a positive constant $C=C(d, \alpha, \beta, A, \varepsilon) \geq 1$ so that for any bounded $b$ satisfying (1.2) with $\|b\|_{\infty} \leq A$ and

$$
j^{b}(x, z) \geq \varepsilon\left(\frac{1}{|z|^{d+\alpha}}+\frac{1}{|z|^{d+\beta}}\right) \quad \text { for a.e. } x, z \in \mathbb{R}^{d}
$$

we have

$$
C^{-1} p_{1}(t, x, y) \leq q^{b}(t, x, y) \leq C p_{1}(t, x, y) \quad \text { for } t \in(0,1] \text { and } x, y \in \mathbb{R}^{d} .
$$

Theorem 1.3 in particular implies that if $b(x, \cdot)$ is a bounded function satisfying (1.2) and (1.19) so that $b(x, z)=0$ for every $x \in \mathbb{R}^{d}$ and $|z| \geq R$ for some $R>0$; or, equivalently if $\mathcal{L}^{b}=$ $\Delta^{\alpha / 2}+\mathcal{S}^{b}$ is a lower order perturbation of $\Delta^{\alpha / 2}$ by finite range non-local operator $\mathcal{S}^{b}$, then the upper bound of the kernel $q^{b}(t, x, y)$ is dominated by $p_{0}(t, x, y)$ for each $(t, x, y) \in(0,1] \times \mathbb{R}^{d} \times \mathbb{R}^{d}$. In fact, we have the following more general result.

Theorem 1.5. For every $A>0$ and $M \geq 1$, there is a constant $C_{5}=C_{5}(d, \alpha, \beta, A, M) \geq 1$ such that for any bounded $b$ satisfying (1.2) with $\|b\|_{\infty} \leq A$ and

$$
M^{-1}|z|^{-(d+\alpha)} \leq j^{b}(x, z) \leq M|z|^{-(d+\alpha)} \quad \text { for a.e. } x, z \in \mathbb{R}^{d},
$$

or equivalently,

$$
-\left(1-M^{-1}\right) \frac{\mathcal{A}(d,-\alpha)}{\mathcal{A}(d,-\beta)}|z|^{\beta-\alpha} \leq b(x, z) \leq(M-1) \frac{\mathcal{A}(d,-\alpha)}{\mathcal{A}(d,-\beta)}|z|^{\beta-\alpha} \quad \text { for a.e. } x, z \in \mathbb{R}^{d},
$$

we have

$$
C_{5}^{-1} p_{0}(t, x, y) \leq q^{b}(t, x, y) \leq C_{5} p_{0}(t, x, y) \quad \text { for } t \in(0,1] \text { and } x, y \in \mathbb{R}^{d}
$$


We can restate some of results from Theorems 1.1, 1.2, 1.3 and 1.5 as follows.

Theorem 1.6. Let $b(x, z)$ be a bounded function on $\mathbb{R}^{d} \times \mathbb{R}^{d}$ satisfying (1.2) and (1.19). For each $x \in \mathbb{R}^{d}$, the martingale problem for $\left(\mathcal{L}^{b}, \mathcal{S}\left(\mathbb{R}^{d}\right)\right)$ with initial value $x$ is well-posed. These martingale problem solutions $\left\{\mathbb{P}_{x}, x \in \mathbb{R}^{d}\right\}$ form a strong Markov process $X^{b}$, which has infinite lifetime and possesses a jointly continuous transition density function $q^{b}(t, x, y)$ with respect to the Lebesgue measure on $\mathbb{R}^{d}$. Moreover, the following holds.

(i) The transition density function $q^{b}(t, x, y)$ can be explicitly constructed as follows. Define $q_{0}^{b}(t, x, y):=p_{0}(t, x, y)$ and

$$
q_{n}^{b}(t, x, y):=\int_{0}^{t} \int_{\mathbb{R}^{d}} q_{n-1}^{b}(t-s, x, z) \mathcal{S}_{z}^{b} p_{0}(s, z, y) d z d s \quad \text { for } n \geq 1 .
$$

There is $\varepsilon>0$ so that $\sum_{n=0}^{\infty} q_{n}^{b}(t, x, y)$ converges absolutely on $(0, \varepsilon] \times \mathbb{R}^{d} \times \mathbb{R}^{d}$ and $q^{b}(t, x, y)=\sum_{n=0}^{\infty} q_{n}^{b}(t, x, y)$ on $(0, \varepsilon] \times \mathbb{R}^{d} \times \mathbb{R}^{d}$.

(ii) $q^{b}(t, x, y)=p_{0}(t, x, y)+\int_{0}^{t} \int_{\mathbb{R}^{d}} q^{b}(t-s, x, z) \mathcal{S}_{z}^{b} p_{0}(s, z, y) d z d s$ on $(0, \infty) \times \mathbb{R}^{d} \times \mathbb{R}^{d}$.

(iii) For every $A>0$ and $\lambda>0$, there are positive constants $c_{k}=c_{k}(d, \alpha, \beta, A), k=1,2,3$ and $c_{k}=c_{k}(d, \alpha, \beta, A, \lambda), k=4, \cdots, 9$, such that for any bounded function $b(x, z)$ on $\mathbb{R}^{d} \times \mathbb{R}^{d}$ satisfying (1.2) and (1.19) with $\|b\|_{\infty} \leq A$,

$$
c_{1} e^{-c_{2} t} \bar{p}_{0}\left(t, c_{3} x, c_{3} y\right) \leq q^{b}(t, x, y) \leq c_{4} e^{c_{5} t} p_{M_{b^{+}, \lambda}}(t, x, y) \quad \text { on }(0, \infty) \times \mathbb{R}^{d} \times \mathbb{R}^{d}
$$

and for any non-negative function $b(x, z)$ on $\mathbb{R}^{d} \times \mathbb{R}^{d}$ satisfying (1.2) with $\|b\|_{\infty} \leq A$,

$$
c_{6} e^{-c_{7} t} p_{m_{b, \lambda}}(t, x, y) \leq q^{b}(t, x, y) \leq c_{8} e^{c_{9} t} p_{M_{b, \lambda}}(t, x, y) \quad \text { on }(0, \infty) \times \mathbb{R}^{d} \times \mathbb{R}^{d} .
$$

(iv) For every $A>0$ and $M \geq 1$, there are positive constants $c_{k}=c_{k}(d, \alpha, \beta, A, M), k=$ $10, \cdots, 13$, such that for any bounded function $b(x, z)$ on $\mathbb{R}^{d} \times \mathbb{R}^{d}$ satisfying (1.2) and (1.27) with $\|b\|_{\infty} \leq A$,

$$
c_{10} e^{-c_{11} t} p_{0}(t, x, y) \leq q^{b}(t, x, y) \leq c_{12} e^{c_{13} t} p_{0}(t, x, y) \quad \text { on }(0, \infty) \times \mathbb{R}^{d} \times \mathbb{R}^{d} .
$$

Remark 1.7. (i) In general, we can not expect $q^{b}$ to have comparable lower and upper bound estimates. The estimates in (1.22) and (1.24) are sharp in the sense that $q^{b}(t, x, y)=p_{0}(t, x, y)$ when $b \equiv 0, q^{b}(t, x, y)=p_{1}(t, x, y)$ when $b \equiv 1$, and $q^{b}(t, x, y)=\bar{p}_{0}(t, x, y)$ when $b(x, z)=0$ for $|z| \leq 1$ and $b(x, z)=-\frac{\mathcal{A}(d,-\alpha)}{\mathcal{A}(d,-\beta)}|z|^{\beta-\alpha}$ for $|z| \geq 1$. Clearly, by (1.7)-(1.8), $p_{0}(t, x, y)$ and $p_{1}(t, x, y)$ are not comparable on $(0,1] \times \mathbb{R}^{d} \times \mathbb{R}^{d}$. We point out that it follows from (1.8) and (1.24) that every $A \geq 1$, there is a constant $\widetilde{C}=\widetilde{C}(d, \alpha, \beta, A) \geq 1$ so that for any non-negative $b$ on $\mathbb{R}^{d} \times \mathbb{R}^{d}$ satisfying (1.2) with $1 / A \leq b(x, z) \leq A$ a.e.

$$
(1 / \widetilde{C}) p_{1}(t, x, y) \leq q^{b}(t, x, y) \leq \widetilde{C} p_{1}(t, x, y) \text { for } t \in(0,1] \text { and } x, y \in \mathbb{R}^{d} .
$$

(ii) Heat kernel estimates for fractional Laplacian $\Delta^{\alpha / 2}$ under gradient perturbation and (possibly non-local) Feynman-Kac perturbation have recently been studied in 6, 9, 10, 29. In both of these cases, under a Kato class condition on the coefficients, the fundamental solution of 
the perturbed operator is always strictly positive and is comparable to the fundamental solution $p_{0}(t, x, y)$ of the fractional Laplacian $\Delta^{\alpha / 2}$ on $(0,1] \times \mathbb{R}^{d} \times \mathbb{R}^{d}$.

The novelty of this paper is on non-local perturbations. The analysis of non-local perturbations with infinite jumping intensity measure is much harder and is in fact very challenging. While the idea of using Duhamel's method (1.11) in the study of operator perturbation is not new, the key is how to implement it to obtain two-sided sharp heat kernel estimates where the lower bound is comparable to the upper bound, and to establish the uniqueness of the fundamental solution. It requires precise estimates on the non-local derivatives of the heat kernel for fractional Laplacian, which turns out to be quite delicate and challenging. To the best of authors' knowledge, this is the first paper on the study of heat kernels under non-local perturbation with infinite jump intensity measure in a systematic way. We emphasize that the function $b(x, z)$ in 1.3 is only measurable. Our Theorems 1.2 and 1.3 reveal some new phenomenon that heat kernels under non-local perturbation $\mathcal{S}^{b}$ are typically unstable. This is is in stark contrast with $\Delta^{\alpha / 2}$ under either gradient (local) perturbations or (possibly non-local) Feynman-Kac perturbations. However, Theorem [1.5] of this paper in particular indicates that the heat kernel estimate for $\Delta^{\alpha / 2}$ is stable under finite range lower order perturbation.

(iii) Kolokoltsov [18] studied heat kernel estimates for symmetric pseudo-differential operators (or stable-like jump diffusions) with smooth symbols. However neither the results nor the approach in [18] applies to our case even when $b(x, z)$ is assumed to be smooth. In addition to the smooth symbol requirement, the operators (1.8)-(1.9) considered in [18 would require $\alpha=\beta$, excluding the case where there are two different stable scales as are considered in this paper. In particular, it does not apply to SDE (1.6). For information on the connection between pseudo-differential operators and discontinuous Markov processes, we refer the reader to [17, 24] and the references therein.

(iv) Martingale problem for non-local operators (with or without elliptic differential operator component) has been studied by many authors. See, e.g., 4, 5, 19, 20, 22, 23, 26, 28, and the references therein. In particular, Komatsu 20] and Mikulevicious-Pragarauskas [22] considered martingale problem for a class of non-local operators that is directly related to $\mathcal{L}^{b}$. In fact, the uniqueness of the martingale problem for $\left(\mathcal{L}^{b}, \mathcal{S}\left(\mathbb{R}^{d}\right)\right)$ stated in Theorem 1.3 above is a direct consequence of [20, Theorem 3], while it follows from [22, Theorem 5] that for any bounded $b$ satisfying (1.2) and (1.19), there is a unique solution to the martingale problem $\left(\mathcal{L}^{b}, C_{c}^{\infty}\left(\mathbb{R}^{d}\right)\right)$. The main contribution of Theorem 1.3 is on the two-sided transition density function estimates for the martingale problem solution $X_{t}^{b}$. We also mention that the well-posedness of martingale problem for $\left(\Delta^{\alpha / 2}+b(x) \cdot \nabla, C_{c}^{\infty}\left(\mathbb{R}^{d}\right)\right)$ with $b(x)$ an $\mathbb{R}^{d}$-valued Kato class function has recently been established in [13].

(v) There are several directions to extend our results. For example, one can replay $\mathcal{A}(d,-\alpha) /|z|^{d+\alpha}$ in (1.1) and $1 /|z|^{d+\beta}$ in (1.3) by the Lévy kernel of pure jump subordinate Brownian motion. This is doable by following the ideas and approach of this paper. Another direction is to consider Laplacian under non-local perturbation; that is, to replace $\Delta^{\alpha / 2}$ in $\mathcal{L}^{b}$ by Laplacian operator $\Delta$. This has recently been carried out in Wang [30.

(v) There are several directions to extend our results. For example, one can replay $\mathcal{A}(d,-\alpha) /|z|^{d+\alpha}$ in (1.1) and $1 /|z|^{d+\beta}$ in (1.3) by the Lévy kernels of pure jump subordinate Brownian motions. This should be doable by following the ideas and approach of this paper. Another direction is to consider Laplacian under non-local perturbation; that is, to replace $\Delta^{\alpha / 2}$ in $\Lambda^{b}$ by Laplacian operator $\Delta$. This has recently been carried out in Wang [30].

The rest of the paper is organized as follows. In Section 2, we derive some estimates on 
$\bar{\Delta}_{x}^{\beta / 2} p_{0}(t, x, y)$ and $\Delta_{x}^{\beta / 2} p_{0}(t, x, y)$ that will be used in later. The existence and uniqueness of the fundamental solution $q^{b}(t, x, y)$ of $\mathcal{L}^{b}$ are given in Section 3 . This is done through a series of lemmas and theorems, which provide more detailed information on $q^{b}(t, x, y)$ and $q_{n}^{b}(t, x, y)$. Theorem 1.1 then follows from these results. We show in Section 4 that the semigroup $\left\{T_{t}^{b} ; t>0\right\}$ associated with $q^{b}(t, x, y)$ is a strongly continuous semigroup in $C_{\infty}\left(\mathbb{R}^{d}\right)$. We then apply HilleYosida-Ray theorem and Courrége's first theorem to establish Theorem 1.2. When $b$ satisfies (1.2), (1.18) and (1.19), $q^{b}(t, x, y)$ determines a conservative Feller process $X^{b}$. We first derive a Lévy system of $X^{b}$ and also prove $\left(X^{b}, \mathbb{P}_{x}\right)$ is the unique solution to the martingale problem for $\left(\mathcal{L}^{b}, \mathcal{S}\left(\mathbb{R}^{d}\right)\right)$ in Section 5 , We next establish, for any given $A>0$, the equi-continuity of $q^{b}(t, x, y)$ on each $[1 / M, M] \times \mathbb{R}^{d} \times \mathbb{R}^{d}$ for any $b$ that satisfies (1.2) with $\|b\|_{\infty} \leq A$. Using this, we can drop the condition (1.18) and establish the Feller process $X^{b}$ with transition density $q^{b}(t, x, y)$ for general bounded $b$ that satisfies (1.2) and (1.19) by approximating it with a sequence of $\left\{k_{n}(x, z), n \geq 1\right\}$ that satisfy (1.2), (1.18) and (1.19). The upper bound estimate for $q^{b}(t, x, y)$ in (1.22) and (1.24) can be obtained from that of $q^{\widehat{b}_{\lambda}}(t, x, y)$ due to the Meyer's construction of $X^{\widehat{b}_{\lambda}}$ from $X^{b}$, where $\widehat{b}_{\lambda}(x, z)=b(x, z) 1_{\{|z| \leq \lambda\}}(z)+b^{+}(x, z) 1_{\{|z|>\lambda\}}(z)$. The lower bound estimates in (1.22) and (1.24) are established by the Lévy system of $X^{b}$ and some probability estimates. Finally, we use the estimates in (1.24) for $b$ with support in $\left\{(x, z) \in \mathbb{R}^{d} \times \mathbb{R}^{d}:|z| \leq 1\right\}$ and the non-local Feynman-Kac perturbation results from [10] to obtain Theorem 1.5.

Throughout this paper, we use the capital letters $C_{1}, C_{2}, \cdots$ to denote constants in the statement of the results, and their labeling will be fixed. The lowercase constants $c_{1}, c_{2}, \cdots$ will denote generic constants used in the proofs, whose exact values are not important and can change from one appearance to another. We will use ":=" to denote a definition. For a differentiable function $f$ on $\mathbb{R}^{d}$, we use $\partial_{i} f$ and $\partial_{i j}^{2} f$ to denote the partial derivatives $\frac{\partial f}{\partial x_{i}}$ and $\frac{\partial^{2} f}{\partial x_{i} \partial x_{j}}$.

\section{Preliminaries}

Suppose that $Y$ is a symmetric $\alpha$-stable process, and $Z$ is a symmetric $\beta$-stable process on $\mathbb{R}^{d}$ that is independent of $Z$. For any $a \geq 0$, we define $Y^{a}$ by $Y_{t}^{a}:=Y_{t}+a^{1 / \beta} Z_{t}$. We will call the process $Y^{a}$ the independent sum of the symmetric $\alpha$-stable process $Y$ and the symmetric $\beta$-stable process $Z$ with weight $a^{1 / \beta}$. The infinitesimal generator of $Y^{a}$ is $\Delta^{\alpha / 2}+a \Delta^{\beta / 2}$. Let $p_{a}(t, x, y)$ denote the transition density of $Y^{a}$ (or equivalently the heat kernel of $\Delta^{\alpha / 2}+a \Delta^{\beta / 2}$ ) with respect to the Lebesgue measure on $\mathbb{R}^{d}$. Recently it is proven in [12] that

$$
p_{1}(t, x, y) \asymp\left(t^{-d / \alpha} \wedge t^{-d / \beta}\right) \wedge\left(\frac{t}{|x-y|^{d+\alpha}}+\frac{t}{|x-y|^{d+\beta}}\right) \quad \text { on }(0, \infty) \times \mathbb{R}^{d} \times \mathbb{R}^{d} .
$$

Unlike the case of the symmetric $\alpha$-stable process $Y:=Y^{0}, Y^{a}$ does not have the stable scaling for $a>0$. Instead, the following approximate scaling property holds : for every $\lambda>0$, $\left\{\lambda^{-1} Y_{\lambda^{\alpha} t}^{a}, t \geq 0\right\}$ has the same distribution as $\left\{Y_{t}^{a \lambda^{(\alpha-\beta)}}, t \geq 0\right\}$. Consequently, for any $\lambda>0$, we have

$$
p_{a \lambda^{(\alpha-\beta)}}(t, x, y)=\lambda^{d} p_{a}\left(\lambda^{\alpha} t, \lambda x, \lambda y\right) \quad \text { for } t>0 \text { and } x, y \in \mathbb{R}^{d} .
$$

In particular, letting $a=1, \lambda=\gamma^{1 /(\alpha-\beta)}$, we get

$$
p_{\gamma}(t, x, y)=\gamma^{d /(\alpha-\beta)} p_{1}\left(\gamma^{\alpha /(\alpha-\beta)} t, \gamma^{1 /(\alpha-\beta)} x, \gamma^{1 /(\alpha-\beta)} y\right) \quad \text { for } t>0 \text { and } x, y \in \mathbb{R}^{d} .
$$


So we deduce from (2.1) that there exists a constant $C>1$ depending only on $d, \alpha$ and $\beta$ such that for every $a>0$ and $(t, x, y) \in(0, \infty) \times \mathbb{R}^{d} \times \mathbb{R}^{d}$

$$
C^{-1} h_{a}(t, x, y) \leq p_{a}(t, x, y) \leq C h_{a}(t, x, y)
$$

where

$$
h_{a}(t, x, y):=\left(t^{-d / \alpha} \wedge(a t)^{-d / \beta}\right) \wedge\left(\frac{t}{|x-y|^{d+\alpha}}+\frac{a t}{|x-y|^{d+\beta}}\right) .
$$

In fact, (2.3) also holds when $a=0$. Observe (see (1.10) ) that for every $A>0$, there is a constant $c=c(d, \alpha, \beta, A) \geq 1$ so that for every $(t, x, y) \in(0,1] \times \mathbb{R}^{d} \times \mathbb{R}^{d}$ and $0 \leq a \leq A$,

$$
c^{-1} t^{-d / \alpha} \wedge\left(\frac{t}{|x-y|^{d+\alpha}}+\frac{a t}{|x-y|^{d+\beta}}\right) \leq h_{a}(t, x, y) \leq c t^{-d / \alpha} \wedge\left(\frac{t}{|x-y|^{d+\alpha}}+\frac{a t}{|x-y|^{d+\beta}}\right)
$$

Recall that $p_{0}(t, x, y)=p_{0}(t, x-y)$ is the transition density function of the symmetric $\alpha$-stable process $Y^{0}$.

Lemma 2.1. There exists a constant $C_{6}=C_{6}(d, \alpha)>0$ such that for every $t>0, x \in \mathbb{R}^{d}$ and $i, j=1, \ldots, d$,

$$
\begin{gathered}
\left|\frac{\partial}{\partial x_{i}} p_{0}(t, x)\right| \leq C_{6} t^{-(d+1) / \alpha}\left(1 \wedge \frac{t^{1 / \alpha}}{|x|}\right)^{d+1+\alpha}, \\
\left|\frac{\partial^{2}}{\partial x_{i} \partial x_{j}} p_{0}(t, x)\right| \leq C_{6} t^{-(d+2) / \alpha}\left(1 \wedge \frac{t^{1 / \alpha}}{|x|}\right)^{d+2+\alpha} .
\end{gathered}
$$

Proof. By [6, Lemma 5], there is a positive constant $c_{1}$ so that for all $t>0$ and $x, y \in \mathbb{R}^{d}$

$$
\left|\nabla_{x} p_{0}(t, x)\right| \leq c_{1}|x|\left(t^{-(d+2) / \alpha} \wedge \frac{t}{|x|^{d+2+\alpha}}\right) \leq c_{1}\left(t^{-(d+1) / \alpha} \wedge \frac{t}{|x|^{d+1+\alpha}}\right) .
$$

That is, the first inequality holds. Let $\eta_{t}(r)$ be the density function of the $\alpha / 2$-stable subordinator at time $t$ and $g(t, x)=(4 \pi t)^{-d / 2} e^{-|x|^{2} / 4 t}$ be the Gaussian kernel on $\mathbb{R}^{d}$. There is a constant $c$ so that $\eta_{t}(r) \leq c t r^{-1-\alpha / 2}$ for all $r, t>0$, see [6, Lemma 5]. Note that

$$
\left|\frac{\partial^{2}}{\partial x_{i} \partial x_{j}} g(s, x)\right| \leq\left(\frac{|x|^{2}}{s^{2}}+\frac{2}{s}\right) g(s, x)=(4 \pi)^{2}|x|^{2} g^{(d+4)}\left(s, x_{1}\right)+8 \pi g^{(d+2)}\left(s, x_{2}\right),
$$

where $x_{1} \in \mathbb{R}^{d+4}$ and $x_{2} \in \mathbb{R}^{d+2}$ with $\left|x_{1}\right|=\left|x_{2}\right|=|x|, g^{(d+2)}\left(s, x_{2}\right)$ and $g^{(d+4)}\left(s, x_{1}\right)$ are the Gaussian kernels on $\mathbb{R}^{d+2}$ and $\mathbb{R}^{d+4}$, respectively. Since $p_{0}(t, x)=\int_{0}^{\infty} g(s, x) \eta_{t}(s) d s$, we have by the dominated convergence theorem that there is a positive constant $c_{2}$ so that for all $t>0$ and $x \in \mathbb{R}^{d}$

$$
\begin{aligned}
\left|\frac{\partial^{2}}{\partial x_{i} \partial x_{j}} p_{0}(t, x)\right| & \leq \int_{0}^{\infty}\left|\frac{\partial^{2}}{\partial x_{i} \partial x_{j}} g(s, x)\right| \eta_{t}(s) d s \\
& \leq(4 \pi)^{2}|x|^{2} p_{0}^{(d+4)}\left(t, x_{1}\right)+8 \pi p_{0}^{(d+2)}\left(t, x_{2}\right) \\
& \leq c_{2}\left(t^{-(d+2) / \alpha} \wedge \frac{t}{|x|^{d+2+\alpha}}\right)
\end{aligned}
$$


where $p_{0}^{(d+2)}\left(t, x_{2}\right)$ and $p_{0}^{(d+4)}\left(t, x_{1}\right)$ are the transition density functions of the symmetric $\alpha$ stable processes in $\mathbb{R}^{d+2}$ and $\mathbb{R}^{d+4}$, respectively. This establishes the second inequality in Lemma 2.1 .

Define for $t>0$ and $x, y \in \mathbb{R}^{d}$, the function

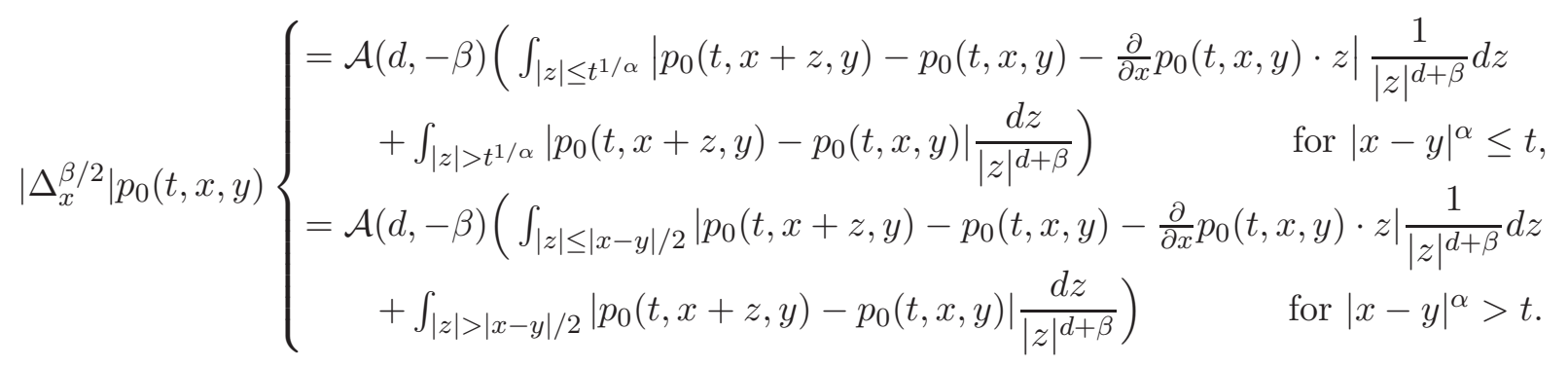

Let

$$
f_{0}(t, x, y):=\left(t^{1 / \alpha} \vee|x-y|\right)^{-(d+\beta)}=t^{-(d+\beta) / \alpha}\left(1 \wedge \frac{t^{1 / \alpha}}{|x-y|}\right)^{d+\beta} .
$$

Lemma 2.2. There exists a constant $C_{7}=C_{7}(d, \alpha, \beta)>0$ such that

$$
\left|\Delta_{x}^{\beta / 2}\right| p_{0}(t, x, y) \leq C_{7} f_{0}(t, x, y) \quad \text { on }(0, \infty) \times \mathbb{R}^{d} \times \mathbb{R}^{d} .
$$

Proof. We only need to prove $\left|\Delta_{x}^{\beta / 2}\right| p_{0}(t, x) \leq C_{7} f_{0}(t, x, 0)$ for all $t>0$ and $x \in \mathbb{R}^{d}$.

(i) We first consider the case $|x|^{\alpha} \leq t$. In this case,

$$
\begin{aligned}
\left|\Delta_{x}^{\beta / 2}\right| p_{0}(t, x)= & \mathcal{A}(d,-\beta) \int_{|z| \leq t^{1 / \alpha}}\left|p_{0}(t, x+z)-p_{0}(t, x)-\frac{\partial}{\partial x} p_{0}(t, x) \cdot z\right| \frac{d z}{|z|^{d+\beta}} \\
& +\mathcal{A}(d,-\beta) \int_{|z| \geq t^{1 / \alpha}}\left|p_{0}(t, x+z)-p_{0}(t, x)\right| \frac{d z}{|z|^{d+\beta}} \\
= & I+I I .
\end{aligned}
$$

Note that by Lemma 2.1,

$$
\sup _{u \in \mathbb{R}^{d}}\left|\frac{\partial^{2}}{\partial u_{i} \partial u_{j}} p_{0}(t, u)\right| \leq C_{6} t^{-(d+2) / \alpha},
$$

and so by Taylor's formula,

$$
I \leq \mathcal{A}(d,-\beta) \sup _{u \in \mathbb{R}^{d}}\left|\frac{\partial^{2}}{\partial u_{i} \partial u_{j}} p_{0}(t, u)\right| \int_{|z| \leq t^{1 / \alpha}} \frac{|z|^{2}}{|z|^{d+\beta}} d z \leq c_{1} t^{-(d+2) / \alpha} t^{(2-\beta) / \alpha} \leq c_{1} t^{-(d+\beta) / \alpha} .
$$

On the other hand, by (1.7)

$I I \leq \mathcal{A}(d,-\beta) \int_{|z| \geq t^{1 / \alpha}}\left(p_{0}(t, x+z)+p_{0}(t, x)\right) \frac{d z}{|z|^{d+\beta}} \leq c_{2} t^{-d / \alpha} \int_{|z| \geq t^{1 / \alpha}} \frac{1}{|z|^{d+\beta}} d z \leq c_{3} t^{-(d+\beta) / \alpha}$.

(ii) Next, we consider the case $|x|^{\alpha} \geq t$. In this case,

$$
\begin{aligned}
\left|\Delta_{x}^{\beta / 2}\right| p_{0}(t, x)= & \mathcal{A}(d,-\beta) \int_{|z| \leq|x| / 2}\left|p_{0}(t, x+z)-p_{0}(t, x)-\frac{\partial}{\partial x} p_{0}(t, x) \cdot z\right| \frac{d z}{|z|^{d+\beta}} \\
& +\mathcal{A}(d,-\beta) \int_{|z| \geq|x| / 2}\left|p_{0}(t, x+z)-p_{0}(t, x)\right| \frac{d z}{|z|^{d+\beta}} \\
= & I+I I .
\end{aligned}
$$


Note that $|x+z| \geq|x| / 2$ for $|z| \leq|x| / 2$. So by Lemma 2.1,

$$
\sup _{|z| \leq|x| / 2}\left|\frac{\partial^{2}}{\partial x_{i} \partial x_{j}} p_{0}(t, x+z)\right| \leq C_{6} \sup _{|z| \leq|x| / 2} t|x+z|^{-(d+2+\alpha)} \leq 2^{(d+2+\alpha)} C_{6} t|x|^{-(d+2+\alpha)} .
$$

Hence, by Taylor's formula

$$
\begin{aligned}
I & \leq \mathcal{A}(d,-\beta) \sup _{|z| \leq|x| / 2}\left|\frac{\partial^{2}}{\partial x_{i} \partial x_{j}} p_{0}(t, x+z)\right| \int_{|z| \leq|x| / 2} \frac{|z|^{2}}{|z|^{d+\beta}} d z \\
& \leq c_{4} t|x|^{-(d+2+\alpha)}|x|^{2-\beta}=c_{4} t|x|^{-(d+\alpha+\beta)} .
\end{aligned}
$$

Noting that $|x|^{\alpha} \geq t$, thus $I \leq c_{4}|x|^{-(d+\beta)}$. On the other hand, note that symmetric $\alpha$-stable process is a subordinate Brownian motion, so $p_{0}(t, x+z) \leq p_{0}(t, x)$ if $|x+z| \geq|x|$ and $p_{0}(t, x) \leq$ $p_{0}(t, x+z)$ if $|x+z| \leq|x|$. Hence, by (1.7) and the condition that $|x|^{\alpha} \geq t$, we obtain

$$
\begin{aligned}
I I & \leq \mathcal{A}(d,-\beta) \int_{|z| \geq|x| / 2,|x+z| \geq|x|} 2 p_{0}(t, x) \frac{d z}{|z|^{d+\beta}}+\mathcal{A}(d,-\beta) \int_{|z| \geq|x| / 2,|x+z| \leq|x|} 2 p_{0}(t, x+z) \frac{d z}{|z|^{d+\beta}} \\
& \leq 2 \mathcal{A}(d,-\beta) p_{0}(t, x) \int_{|z| \geq|x| / 2} \frac{d z}{|z|^{d+\beta}}+2^{d+1+\beta} \mathcal{A}(d,-\beta)|x|^{-(d+\beta)} \int_{z \in \mathbb{R}^{d}} p_{0}(t, x+z) d z \\
& \leq c_{5} t|x|^{-(d+\alpha)}|x|^{-\beta}+2^{d+1+\beta} \mathcal{A}(d,-\beta)|x|^{-(d+\beta)} \leq c_{6}|x|^{-(d+\beta)} .
\end{aligned}
$$

This establishes the lemma.

In order to get the upper bound estimates in (1.16) in terms of weight $M_{b, \lambda}$ rather than $\|b\|_{\infty}$, we define, for $t>0, \lambda>0$ and $x, y \in \mathbb{R}^{d}$, the function

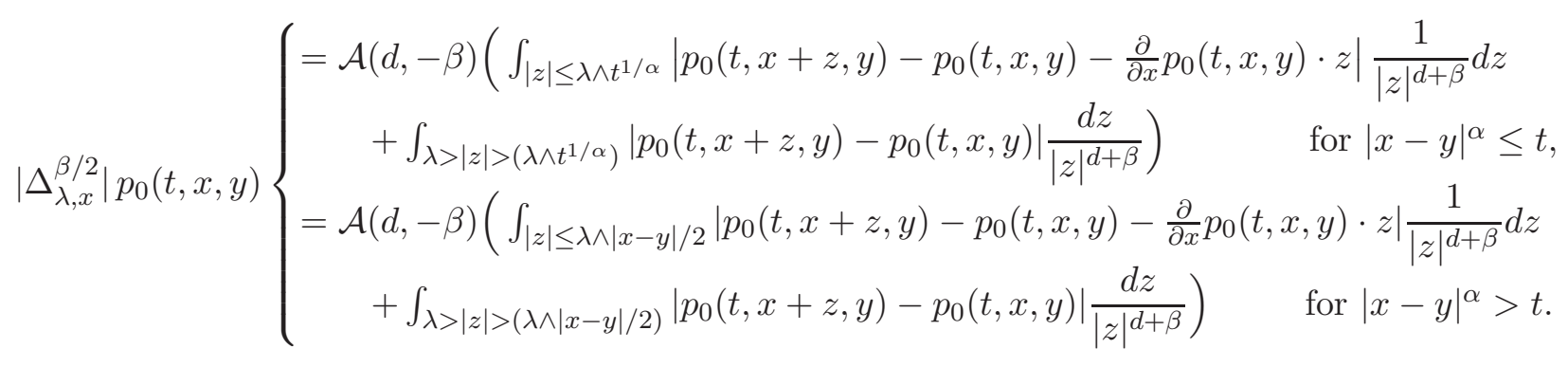

Observe that

$$
\left|\Delta_{\lambda, x}^{\beta / 2}\right| p_{0}(t, x, y)|\leq| \Delta_{x}^{\beta / 2} \mid p_{0}(t, x, y)
$$

Set

$$
f_{0, \lambda}(t, x, y)= \begin{cases}t^{-(d+\beta) / \alpha} & \text { when }|x-y| \leq t^{1 / \alpha} \\ |x-y|^{-(d+\beta)} \mathbb{1}_{\{|x-y| \leq \lambda\}}+|x-y|^{-(d+\alpha)} \mathbb{1}_{\{|x-y|>\lambda\}} & \text { when }|x-y|>t^{1 / \alpha}\end{cases}
$$

Observe that when $\lambda=\infty, f_{0, \infty}$ is just the function $f_{0}$ defined in (2.5).

Lemma 2.3. For each $\lambda>0$ and $T>0$, there exists a constant $C_{8}=C_{8}(d, \alpha, \beta, \lambda, T)>0$ such that

$$
\left|\Delta_{\lambda, x}^{\beta / 2}\right| p_{0}(t, x, y) \leq C_{8} f_{0, \lambda}(t, x, y) \quad \text { on }(0, T] \times \mathbb{R}^{d} \times \mathbb{R}^{d} .
$$


Proof. (i) We first consider the case $|x-y|^{\alpha} \leq t$. Note that

$$
\left|\Delta_{\lambda, x}^{\beta / 2}\right| p_{0}(t, x, y) \leq\left|\Delta_{x}^{\beta / 2}\right| p_{0}(t, x, y) .
$$

Hence, by the first part $(i)$ in the proof of Lemma 2.2, there exists a positive constant $c_{1}$ so that

$$
\left|\Delta_{\lambda, x}^{\beta / 2}\right| p_{0}(t, x, y) \leq c_{1} t^{-(d+\beta) / \alpha} .
$$

(ii) Next, we consider the case $|x-y|^{\alpha}>t$. In this case

$$
\begin{aligned}
\left|\Delta_{\lambda, x}^{\beta / 2}\right| p_{0}(t, x, y) \leq & \mathcal{A}(d,-\beta) \int_{|z| \leq|x-y| / 2}\left|p_{0}(t, x+z, y)-p_{0}(t, x, y)-\frac{\partial}{\partial x} p_{0}(t, x, y) \cdot z\right| \frac{d z}{|z|^{d+\beta}} \\
& +\mathcal{A}(d,-\beta) \int_{\lambda \geq|z| \geq(\lambda \wedge|x-y| / 2)}\left|p_{0}(t, x+z, y)-p_{0}(t, x, y)\right| \frac{d z}{|z|^{d+\beta}} \\
= & I+I I .
\end{aligned}
$$

By (2.7), there is a positive constant $c_{2}$ so that

$$
I \leq c_{2} t|x-y|^{-(d+\alpha+\beta)} \leq c_{3}\left(|x-y|^{-(d+\beta)} \mathbb{1}_{\{|x-y| \leq 2 \lambda\}}+|x-y|^{-(d+\alpha)} \mathbb{1}_{\{|x-y|>2 \lambda\}}\right) .
$$

Here the last inequality holds since $t|x-y|^{-(d+\alpha+\beta)} \leq T(2 \lambda)^{-\beta}|x-y|^{-(d+\alpha)}$ when $|x-y|>2 \lambda$ and $t|x-y|^{-(d+\alpha+\beta)} \leq|x-y|^{-(d+\beta)}$ due to $|x-y|^{\alpha} \geq t$.

It is clear that $I I=0$ if $|x-y|>2 \lambda$. On the other hand, if $|x-y| \leq 2 \lambda$, then there exists a positive constant $c_{4}$ so that $I I \leq c_{4}|x-y|^{-(d+\beta)}$ by (2.8). Finally, we note that $|x-y|^{-(d+\beta)} \asymp|x-y|^{-(d+\alpha)}$ for $\lambda<|x-y| \leq 2 \lambda$. This establishes the lemma.

For each $\lambda>0$ and $a \geq 0$, we extend the definition of $f_{0, \lambda}(t, x, y)$ to define

$$
f_{a, \lambda}(t, x, y):=\left\{\begin{array}{l}
t^{-(d+\beta) / \alpha} \\
\text { when }|x-y| \leq t^{1 / \alpha} \\
|x-y|^{-(d+\beta)} \mathbb{1}_{\{|x-y| \leq \lambda\}}+\left(|x-y|^{-(d+\alpha)}+a|x-y|^{-(d+\beta)}\right) \mathbb{1}_{\{|x-y|>\lambda\}} \\
\text { when }|x-y|>t^{1 / \alpha}
\end{array}\right.
$$

Note that $f_{a, \infty}(t, x, y)=f_{0}(t, x, y)$.

Lemma 2.4. For each $\lambda>0$, there is a constant $C_{9}=C_{9}(d, \alpha, \beta, \lambda)>0$ such that for every $a \in[0,1]$,

$$
\int_{0}^{t} \int_{\mathbb{R}^{d}} f_{a, \lambda}(s, z, y) d z d s \leq C_{9}\left(t^{1-\beta / \alpha}+t\right), \quad t \in(0, \infty), y \in \mathbb{R}^{d} .
$$

Proof. By the definition of $f_{a, \lambda}$,

$$
\begin{aligned}
& \int_{0}^{t} \int_{\mathbb{R}^{d}} f_{a, \lambda}(s, z, y) d z d s \\
\leq & \int_{0}^{t} \int_{|y-z| \leq s^{1 / \alpha}} s^{-(d+\beta) / \alpha} d z d s+\int_{0}^{t} \int_{\lambda \geq|y-z|>s^{1 / \alpha}} \frac{1}{|y-z|^{d+\beta}} d z d s \\
& +\int_{0}^{t} \int_{|y-z| \geq \lambda}\left(|y-z|^{-(d+\alpha)}+|y-z|^{-(d+\beta)}\right) d z d s \\
\leq & c_{1} \int_{0}^{t}\left(s^{-\beta / \alpha}+1\right) d s \leq c_{2}\left(t^{1-\beta / \alpha}+t\right) .
\end{aligned}
$$


For every $a \geq 0$, define

$$
g_{a}(t, x, y)= \begin{cases}t^{-d / \alpha} & \text { when }|x-y| \leq t^{1 / \alpha} \\ \frac{t}{|x-y|^{d+\alpha}}+\frac{a t}{|x-y|^{d+\beta}} & \text { when }|x-y|>t^{1 / \alpha}\end{cases}
$$

Observe that

$$
\int_{\mathbb{R}^{d}} g_{a}(t, x, y) d y \asymp 1+a t^{1-\beta / \alpha} \quad \text { on }(0, \infty) \times \mathbb{R}^{d} .
$$

Recall that $p_{a}(t, x, y)$ is the heat kernel of the operator $\Delta^{\alpha / 2}+a \Delta^{\beta / 2}$. Moreover, in view of (1.10),

$$
g_{a}(t, x, y) \asymp p_{a}(t, x, y) \quad \text { on }(0,1] \times \mathbb{R}^{d} \times \mathbb{R}^{d} .
$$

Lemma 2.5. For each $\lambda>0$ and $T>0$, there exists $C_{10}=C_{10}(d, \alpha, \beta, \lambda, T)>0$ such that for every $a \in[0,1]$ and all $t \in(0, T], x, y \in \mathbb{R}^{d}$,

$$
\int_{0}^{t} \int_{\mathbb{R}^{d}} g_{a}(t-s, x, z) f_{a, \lambda}(s, z, y) d z d s \leq C_{10} g_{a}(t, x, y) .
$$

Proof. Denote by $I=\int_{0}^{t} \int_{\mathbb{R}^{d}} g_{a}(t-s, x, z) f_{a, \lambda}(s, z, y) d z d s$.

(i) Suppose that $|x-y| \leq t^{1 / \alpha}$. Then

$$
\begin{aligned}
I= & \int_{0}^{t} \int_{|x-z| \leq 2 t^{1 / \alpha}} g_{a}(t-s, x, z) f_{a, \lambda}(s, z, y) d z d s \\
& +\int_{0}^{t} \int_{|x-z|>2 t^{1 / \alpha}} g_{a}(t-s, x, z) f_{a, \lambda}(s, z, y) d z d s \\
= & : I_{1}+I_{2} .
\end{aligned}
$$

We write $I_{1}$ as

$$
\begin{aligned}
I_{1}= & \int_{0}^{t / 2} \int_{|x-z| \leq 2 t^{1 / \alpha}} g_{a}(t-s, x, z) f_{a, \lambda}(s, z, y) d z d s \\
& \quad+\int_{t / 2}^{t} \int_{|x-z| \leq 2 t^{1 / \alpha}} g_{a}(t-s, x, z) f_{a, \lambda}(s, z, y) d z d s \\
= & I_{11}+I_{12} .
\end{aligned}
$$

If $s \in(0, t / 2)$, then $t-s \in(t / 2, t)$. In this case, $g_{a}(t-s, x, z) \leq c_{1} t^{-d / \alpha}$ when $|x-z| \leq 2 t^{1 / \alpha}$ by (2.12). Hence, by Lemma 2.4.

$$
I_{11} \leq c_{1} t^{-d / \alpha} \int_{0}^{t} \int_{\mathbb{R}^{d}} f_{a, \lambda}(s, z, y) d z d s \leq c_{2}\left(T^{1-\beta / \alpha}+T\right) t^{-d / \alpha} .
$$

When $s \in[t / 2, t]$, since $|x-y| \leq t^{1 / \alpha}$ and $|x-z| \leq 2 t^{1 / \alpha},|y-z| \leq 3 t^{1 / \alpha} \leq 3(2 s)^{1 / \alpha}$. Thus $f_{a, \lambda}(s, z, y) \leq c_{3} s^{-(d+\beta) / \alpha} \leq 2^{(d+\beta) / \alpha} c_{3} t^{-(d+\beta) / \alpha}$. Hence,

$$
I_{12} \leq 2^{(d+\beta) / \alpha} c_{3} t^{-(d+\beta) / \alpha} \int_{0}^{t} \int_{\mathbb{R}^{d}} g_{a}(t-s, x, z) d z d s \leq c_{4} T^{1-\beta / \alpha}\left(1+T^{1-\beta / \alpha}\right) t^{-d / \alpha} .
$$


Next we consider $I_{2}$. Noting that $|x-z|>2 t^{1 / \alpha}$, so we have by (2.12) and Lemma 2.4.

$$
\begin{aligned}
I_{2} & \leq c_{5} \int_{0}^{t} \int_{|x-z|>2 t^{1 / \alpha}}\left(\frac{t-s}{|x-z|^{d+\alpha}}+\frac{t-s}{|x-z|^{d+\beta}}\right) f_{a, \lambda}(s, z, y) d z d s \\
& \leq c_{6} t^{-d / \alpha}\left(1+t^{1-\beta / \alpha}\right) \int_{0}^{t} \int_{\mathbb{R}^{d}} f_{a, \lambda}(s, z, y) d z d s \\
& \leq c_{7}\left(1+T^{1-\beta / \alpha}\right)\left(T^{1-\beta / \alpha}+T\right) t^{-d / \alpha} .
\end{aligned}
$$

We thus conclude from the above that there is a $c_{8}=c_{8}(d, \alpha, \beta, \lambda, T)>0$ such that $I \leq c_{8} t^{-d / \alpha}$ for every $t \in(0, T]$ whenever $|x-y| \leq t^{1 / \alpha}$.

(ii) Next assume that $|x-y|>t^{1 / \alpha}$. Then

$$
\begin{aligned}
I= & \int_{0}^{t} \int_{|x-z| \leq|x-y| / 2} g_{a}(t-s, x, z) f_{a, \lambda}(s, z, y) d z d s \\
& \quad+\int_{0}^{t} \int_{|x-z|>|x-y| / 2} g_{a}(t-s, x, z) f_{a, \lambda}(s, z, y) d z d s \\
=: & I_{1}+I_{2} .
\end{aligned}
$$

If $|x-z| \leq|x-y| / 2$, then $|y-z| \geq|x-y| / 2>t^{1 / \alpha} / 2$. Hence, there is a constant $c_{9}$ so that

$$
f_{a, \lambda}(s, z, y) \leq c_{9}\left(|x-y|^{-(d+\alpha)}+a|x-y|^{-(d+\beta)}\right)
$$

for $s \in(0, t)$. Therefore,

$$
\begin{aligned}
I_{1} & \leq c_{9}\left(|x-y|^{-(d+\alpha)}+a|x-y|^{-(d+\beta)}\right) \cdot \int_{0}^{t} \int_{\mathbb{R}^{d}} g_{a}(t-s, x, z) d z d s \\
& \leq c_{10}\left(1+T^{1-\beta / \alpha}\right)\left(\frac{t}{|x-y|^{d+\alpha}}+\frac{a t}{|x-y|^{d+\beta}}\right) .
\end{aligned}
$$

If $|x-z|>|x-y| / 2$, then $|x-z|>t^{1 / \alpha} / 2$. Hence $g_{a}(t-s, x, z) \leq c_{11}\left(\frac{t}{|x-y|^{d+\alpha}}+\frac{a t}{|x-y|^{d+\beta}}\right)$ by (2.12). Thus by Lemma 2.4, we obtain

$$
\begin{aligned}
I_{2} & \leq c_{11}\left(\frac{t}{|x-y|^{d+\alpha}}+\frac{a t}{|x-y|^{d+\beta}}\right) \int_{0}^{t} \int_{\mathbb{R}^{d}} f_{a, \lambda}(s, z, y) d z d s \\
& \leq c_{12}\left(T^{1-\beta / \alpha}+T\right)\left(\frac{t}{|x-y|^{d+\alpha}}+\frac{a t}{|x-y|^{d+\beta}}\right) .
\end{aligned}
$$

This completes the proof of the Lemma.

\section{Fundamental solution}

Throughout the rest of this paper, $b(x, z)$ is a bounded function on $\mathbb{R}^{d} \times \mathbb{R}^{d}$ satisfying condition (1.2). Recall the definition of the non-local operator $\mathcal{S}^{b}$ from (1.3). Let $\left|q^{b}\right|_{0}(t, x, y)=p_{0}(t, x, y)$, and define for each $n \geq 1$,

$$
\left|q^{b}\right|_{n}(t, x, y)=\int_{0}^{t} \int_{\mathbb{R}^{d}}\left|q^{b}\right|_{n-1}(t-s, x, z)\left|\mathcal{S}_{z}^{b} p_{0}(s, z, y)\right| d z d s .
$$


For each $\lambda>0$, define

$$
b_{\lambda}(x, z)=b(x, z) 1_{\{|z|>\lambda\}}(z) .
$$

In view of (1.7), there exists a constant $C_{11}=C_{11}(d, \alpha, \beta)>0$ such that $p_{0}(t, x, y) \leq$ $C_{11} g_{a}(t, x, y)$ for all $t>0, a \in[0,1]$ and $x, y \in \mathbb{R}^{d}$, where $g_{a}$ is the function defined by (2.12). On the other hand, note that

$$
\begin{aligned}
\left|\mathcal{S}^{b} f(x)\right|= & \left|\mathcal{A}(d,-\beta) \int_{\mathbb{R}^{d}}\left(f(x+z)-f(x)-\langle\nabla f(x), z\rangle \mathbb{1}_{\{|z| \leq \lambda\}}\right) \frac{b(x, z)}{|z|^{d+\beta}} d z\right| \\
\leq & \left|\mathcal{A}(d,-\beta) \int_{|z| \leq \lambda}(f(x+z)-f(x)-\langle\nabla f(x), z\rangle) \frac{b(x, z)}{|z|^{d+\beta}} d z\right| \\
& +\left|\mathcal{A}(d,-\beta) \int_{\mathbb{R}^{d}}(f(x+z)-f(x)) \frac{b_{\lambda}(x, z)}{|z|^{d+\beta}} d z\right| \\
\leq & \|b\|_{\infty} \cdot\left|\Delta_{\lambda, x}^{\beta / 2}\right| f(x)+\left\|b_{\lambda}\right\|_{\infty} \cdot\left|\Delta_{x}^{\beta / 2}\right| f(x)
\end{aligned}
$$

where $\left|\Delta_{\lambda, x}^{\beta / 2}\right| f(x)$ is defined in the similar way as $\left|\Delta_{\lambda, x}^{\beta / 2}\right| p_{0}(t, x, y)$. Then by Lemma 2.2 and Lemma 2.3, for every $A>0, \lambda>0$ and $T>0$ and every bounded function $b$ with $\|b\|_{\infty} \leq A$,

$$
\begin{aligned}
\left|\mathcal{S}_{z}^{b} p_{0}(t, z, y)\right| & \leq\|b\|_{\infty} \cdot\left|\Delta_{\lambda, z}^{\beta / 2}\right| p_{0}(t, z, y)+\left\|b_{\lambda}\right\|_{\infty} \cdot\left|\Delta_{z}^{\beta / 2}\right| p_{0}(t, z, y) \\
& \leq C_{8} A f_{0, \lambda}(t, z, y)+C_{7} M_{b, \lambda} f_{0}(t, z, y) \\
& \leq\left(C_{7}+C_{8}\right) A f_{M_{b, \lambda} / A, \lambda}(t, z, y), \quad t \in(0, T] .
\end{aligned}
$$

Here recall that $M_{b, \lambda}=\operatorname{esssup}_{x, z \in \mathbb{R}^{d},|z|>\lambda}|b(x, z)|, f_{a, \lambda}$ is the function defined in (2.10). The above estimate is a refinement of Lemma 2.2. The latter corresponds to the case where $\lambda=\infty$.

Lemma 3.1. For each $\lambda>0, A>0$ and $T>0$ and every bounded function $b$ on $\mathbb{R}^{d} \times \mathbb{R}^{d}$ satisfying condition (1.2) with $\|b\|_{\infty} \leq A$,

$$
\left|q^{b}\right|_{n}(t, x, y) \leq C_{11}\left(A\left(C_{7}+C_{8}\right) C_{10}\right)^{n} g_{M_{b, \lambda} / A}(t, x, y)<\infty, \quad t \in(0, T], x, y \in \mathbb{R}^{d} .
$$

Proof. We prove this lemma by induction. Since $p_{0}(t, x, y) \leq C_{11} g_{M_{b, \lambda} / A}(t, x, y)$ and $M_{b, \lambda} / A \leq$ 1 , in view of Lemma 2.5 and (3.1), (3.2) clearly holds for $n=1$. Suppose that (3.2) holds for $n=j \geq 1$. Then by Lemma 2.5 and (3.1),

$$
\begin{aligned}
& \left|q^{b}\right|_{j+1}(t, x, y) \\
\leq & C_{11}\left(A\left(C_{7}+C_{8}\right) C_{10}\right)^{j} \int_{0}^{t} \int_{\mathbb{R}^{d}} g_{M_{b, \lambda} / A}(t-s, x, z)\left|\mathcal{S}_{z}^{b} p_{0}(s, z, y)\right| d z d s \\
\leq & C_{11}\left(A\left(C_{7}+C_{8}\right) C_{10}\right)^{j}\left(C_{7}+C_{8}\right) A \int_{0}^{t} \int_{\mathbb{R}^{d}} g_{M_{b, \lambda} / A}(t-s, x, z) f_{M_{b, \lambda} / A, \lambda}(s, z, y) d z d s \\
\leq & C_{11}\left(A\left(C_{7}+C_{8}\right) C_{10}\right)^{j+1} g_{M_{b, \lambda} / A}(t, x, y)
\end{aligned}
$$

for $t \in(0, T]$ and $x, y \in \mathbb{R}^{d}$. This proves that (3.2) holds for $n=j+1$ and thus for every $n \geq 1$.

Now we define $q_{n}^{b}:(0, \infty) \times \mathbb{R}^{d} \times \mathbb{R}^{d} \rightarrow \mathbb{R}$ as follows. For $t>0$ and $x, y \in \mathbb{R}^{d}$, let $q_{0}^{b}(t, x, y)=p_{0}(t, x, y)$, and for each $n \geq 1$, define

$$
q_{n}^{b}(t, x, y)=\int_{0}^{t} \int_{\mathbb{R}^{d}} q_{n-1}^{b}(t-s, x, z) \mathcal{S}_{z}^{b} p_{0}(s, z, y) d z d s .
$$

Clearly by Lemma 3.1, each $q_{n}^{b}(t, x, y)$ is well defined. 
Lemma 3.2. For every $n \geq 0, q_{n}^{b}(t, x, y)$ is jointly continuous on $(0, \infty) \times \mathbb{R}^{d} \times \mathbb{R}^{d}$.

Proof. We prove it by induction. Clearly $q_{0}^{b}(t, x, y)$ is continuous on $(0, \infty) \times \mathbb{R}^{d} \times \mathbb{R}^{d}$. Suppose that $q_{n}^{b}(t, x, y)$ is continuous on $(0, \infty) \times \mathbb{R}^{d} \times \mathbb{R}^{d}$. For every $M \geq 2$, it follows from (3.1), Lemma 3.1 and the dominated convergence theorem that for $\varepsilon<1 /(2 M)$,

$$
(t, x, y) \mapsto \int_{\varepsilon}^{t-\varepsilon} \int_{\mathbb{R}^{d}} q_{n}^{b}(t-s, x, z) \mathcal{S}_{z}^{b} p_{0}(s, z, y) d z d s
$$

is jointly continuous on $[1 / M, M] \times \mathbb{R}^{d} \times \mathbb{R}^{d}$. On the other hand, it follows from (3.1) and (2.13) that

$$
\begin{aligned}
& \sup _{t \in[1 / M, M]} \sup _{x, y} \int_{t-\varepsilon}^{t} \int_{\mathbb{R}^{d}} g_{M_{b, \lambda}}(t-s, x, z)\left|\mathcal{S}_{z}^{b} p_{0}(s, z, y)\right| d z d s \\
\leq & c_{1} A\left(\sup _{t \in[1 / M, M]}\left[(t-\varepsilon)^{-(d+\beta) / \alpha}+(t-\varepsilon)^{-(d+\alpha) / \alpha}\right]\right) \sup _{x \in \mathbb{R}^{d}} \sup _{t \in[1 / M, M]} \int_{t-\varepsilon}^{t} \int_{\mathbb{R}^{d}} g_{M_{b, \lambda}}(t-s, x, z) d z d s \\
\leq & c_{2} A(2 M)^{(d+\alpha) / \alpha} \int_{0}^{\varepsilon}\left(1+r^{1-\beta / \alpha}\right) d r \\
\leq & c_{3} A(2 M)^{(d+\alpha) / \alpha} \varepsilon
\end{aligned}
$$

which goes to zero as $\varepsilon \rightarrow 0$; while by (3.1) and (2.11), there exist $c_{4}$ and $c_{5}$ such that

$$
\begin{aligned}
& \sup _{t \in[1 / M, M]} \sup _{x, y} \int_{0}^{\varepsilon} \int_{\mathbb{R}^{d}} g_{M_{b, \lambda}}(t-s, x, z)\left|\mathcal{S}_{z}^{b} p_{0}(s, z, y)\right| d z d s \\
\leq & c_{4}\left(\sup _{t \in[1 / M, M]}(t-\varepsilon)^{-d / \alpha}\right) \sup _{y \in \mathbb{R}^{d}} \int_{0}^{\varepsilon} \int_{\mathbb{R}^{d}}\left|\mathcal{S}_{z}^{b} p_{0}(s, z, y)\right| d z d s \\
\leq & c_{5}(2 M)^{d / \alpha}\|b\|_{\infty} \varepsilon^{1-\beta / \alpha} \rightarrow 0
\end{aligned}
$$

as $\varepsilon \rightarrow 0$. We conclude from Lemma 3.1 and the above argument that

$$
q_{n+1}^{b}(t, x, y)=\int_{0}^{t} \int_{\mathbb{R}^{d}} q_{n}^{b}(t-s, x, z) \mathcal{S}_{z}^{b} p_{0}(s, z, y) d z d s
$$

is jointly continuous in $(t, x, y) \in[1 / M, M] \times \mathbb{R}^{d} \times \mathbb{R}^{d}$ and so in $(t, x, y) \in(0, \infty) \times \mathbb{R}^{d} \times \mathbb{R}^{d}$. This completes the proof of the lemma.

Recall $f_{0}(t, x, y)$ is the function defined in (2.5) and

$$
\left|\Delta_{x}^{\beta / 2}\right| p_{0}(t, x, y) \leq C_{7} f_{0}(t, x, y) \quad \text { on }(0, \infty) \times \mathbb{R}^{d} \times \mathbb{R}^{d} .
$$

Lemma 3.3. There is a constant $C_{12}=C_{12}(d, \alpha, \beta)>0$ so that for every $A>0$ and every bounded function $b$ on $\mathbb{R}^{d} \times \mathbb{R}^{d}$ with $\|b\|_{\infty} \leq A$ and for every integer $n \geq 0$ and $\varepsilon>0$,

$$
\left|\int_{\left\{z \in \mathbb{R}^{d}:|z|>\varepsilon\right\}}\left(q_{n}^{b}(t, x+z, y)-q_{n}^{b}(t, x, y)\right) \frac{\mathcal{A}(d,-\beta) b(x, z)}{|z|^{d+\beta}} d z\right| \leq\left(C_{12} A\right)^{n+1} f_{0}(t, x, y)
$$


for $(t, x, z) \in(0,1] \times \mathbb{R}^{d} \times \mathbb{R}^{d}$, and $\mathcal{S}_{x}^{b} q_{n}^{b}(t, x, y)$ exists pointwise for $(t, x, z) \in(0,1] \times \mathbb{R}^{d} \times \mathbb{R}^{d}$ in the sense of (1.5) with

$$
\mathcal{S}_{x}^{b} q_{n+1}^{b}(t, x, y)=\int_{0}^{t} \int_{\mathbb{R}^{d}} \mathcal{S}_{x}^{b} q_{n}^{b}(t-s, x, z) \mathcal{S}_{z}^{b} p_{0}(s, z, y) d z d s
$$

and

$$
\left|\mathcal{S}_{x}^{b} q_{n}^{b}(t, x, y)\right| \leq\left(C_{12} A\right)^{n+1} f_{0}(t, x, y) \quad \text { on }(0,1] \times \mathbb{R}^{d} \times \mathbb{R}^{d}
$$

Moreover,

$$
q_{n+1}^{b}(t, x, y)=\int_{0}^{t} \int_{\mathbb{R}^{d}} p_{0}(t-s, x, z) \mathcal{S}_{z}^{b} q_{n}^{b}(s, z, y) d z d s \quad \text { for }(t, x, y) \in(0,1] \times \mathbb{R}^{d} \times \mathbb{R}^{d} .
$$

Proof. Let $q(t, x, y)$ denote the transition density function of the symmetric $\beta$-stable process on $\mathbb{R}^{d}$. Then by (1.7) but with $\beta$ in place of $\alpha$, we have

$$
q(t, x, y) \asymp t^{-d / \beta}\left(1 \wedge \frac{t^{1 / \beta}}{|x-y|}\right)^{d+\beta} \quad \text { on }(0, \infty) \times \mathbb{R}^{d} \times \mathbb{R}^{d} .
$$

Observe that (2.5) and (3.9) yield

$$
f_{0}(t, x, y) \asymp t^{-\beta / \alpha} q\left(t^{\beta / \alpha}, x, y\right) \quad \text { on }(0, \infty) \times \mathbb{R}^{d} \times \mathbb{R}^{d} .
$$

Hence on $(0, \infty) \times \mathbb{R}^{d} \times \mathbb{R}^{d}$,

$$
\begin{aligned}
& \int_{0}^{t} \int_{\mathbb{R}^{d}} f_{0}(t-s, x, z) f_{0}(s, z, y) d s d z \\
\asymp & \int_{0}^{t}(t-s)^{-\beta / \alpha} s^{-\beta / \alpha}\left(\int_{\mathbb{R}^{d}} q\left((t-s)^{\beta / \alpha}, x, z\right) q\left(s^{\beta / \alpha}, z, y\right) d z\right) d s \\
= & \int_{0}^{t}(t-s)^{-\beta / \alpha} s^{-\beta / \alpha} q\left((t-s)^{\beta / \alpha}+s^{\beta / \alpha}, x, y\right) d s \\
\asymp & q\left(t^{\beta / \alpha}, x, y\right) \int_{0}^{t}(t-s)^{-\beta / \alpha} s^{-\beta / \alpha} d s \\
= & q\left(t^{\beta / \alpha}, x, y\right) t^{1-(2 \beta / \alpha)} \int_{0}^{1}(1-u)^{-\beta / \alpha} u^{-\beta / \alpha} d u \\
\asymp & t^{1-\beta / \alpha} f_{0}(t, x, y) .
\end{aligned}
$$

In the second $\asymp$ above, we used the fact that

$$
(t / 2)^{\beta / \alpha} \leq(t-s)^{\beta / \alpha}+s^{\beta / \alpha} \leq 2 t^{\beta / \alpha} \quad \text { for every } s \in(0, t)
$$

and the estimate (3.9), while in the last equality, we used a change of variable $s=t u$. So there is a constant $c_{1}=c_{1}(d, \alpha, \beta)>0$ so that

$$
\int_{0}^{t} \int_{\mathbb{R}^{d}} f_{0}(t-s, x, z) f_{0}(s, z, y) d s d z \leq c_{1} f_{0}(t, x, y) \quad \text { for every } t \in(0,1] \text { and } x, y \in \mathbb{R}^{d} .
$$

By increasing the value of $c_{1}$ if necessary, we may and do assume that $c_{1}$ is larger than 1 . 
We now proceed by induction. Let $C_{12}:=c_{1} C_{7}$. Note that

$$
\left|\mathcal{S}_{x}^{b} p_{0}(t, x, y)\right| \leq A\left|\Delta_{x}^{\beta / 2}\right| p_{0}(t, x, y) \leq C_{7} A f_{0}(t, x, y) .
$$

When $n=0$, (3.8) holds by definition. By Lemma 2.2, (3.5) and (3.7) hold for $n=0$. Suppose that (3.5) and (3.7) hold for $n=j$. Then for every $\varepsilon>0$, by the definition of $q_{j+1}^{b}$, Lemma 3.1, (3.11) and Fubini's theorem,

$$
\begin{gathered}
\int_{\left\{w \in \mathbb{R}^{d}:|\omega|>\varepsilon\right\}}\left(q_{j+1}^{b}(t, x+w, y)-q_{j+1}^{b}(t, x, y)\right) \frac{\mathcal{A}(d,-\beta) b(x, w)}{|w|^{d+\beta}} d w \\
=\int_{0}^{t} \int_{\mathbb{R}^{d}}\left(\int_{\left\{w \in \mathbb{R}^{d}:|w|>\varepsilon\right\}}\left(q_{j}^{b}(t-s, x+w, z)-q_{j}^{b}(t-s, x, z)\right) \frac{\mathcal{A}(d,-\beta) b(x, w)}{|w|^{d+\beta}} d w\right) \\
\quad \times \mathcal{S}_{z}^{b} p_{0}(s, z, y) d z d s
\end{gathered}
$$

and so

$$
\begin{aligned}
& \left|\int_{\left\{w \in \mathbb{R}^{d}:|w|>\varepsilon\right\}}\left(q_{j+1}^{b}(t, x+w, y)-q_{j+1}^{b}(t, x, y)\right) \frac{\mathcal{A}(d,-\beta) b(x, w)}{|w|^{d+\beta}} d w\right| \\
\leq & \int_{0}^{t} \int_{\mathbb{R}^{d}}\left(C_{12} A\right)^{j+1} f_{0}(t-s, x, z)\left|\mathcal{S}_{z}^{b} p_{0}(s, z, y)\right| d z d s \\
\leq & \int_{0}^{t} \int_{\mathbb{R}^{d}}\left(C_{12} A\right)^{j+1} f_{0}(t-s, x, z) C_{7} A f_{0}(s, z, y) d z d s \\
\leq & \left(C_{12} A\right)^{j+2} f_{0}(t, x, y) .
\end{aligned}
$$

By (3.13) and Lebesgue dominated convergence theorem, we conclude that

$$
\begin{aligned}
& S_{x}^{b} q_{j+1}^{b}(t, x, y) \\
:= & \lim _{\varepsilon \rightarrow 0} \int_{\left\{w \in \mathbb{R}^{d}:|w|>\varepsilon\right\}}\left(q_{j+1}^{b}(t, x+w, y)-q_{j+1}^{b}(t, x, y)\right) \frac{\mathcal{A}(d,-\beta) b(x, w)}{|w|^{d+\beta}} d w \\
= & \int_{0}^{t} \int_{\mathbb{R}^{d}}\left(\lim _{\varepsilon \rightarrow 0} \int_{\left\{w \in \mathbb{R}^{d}:|w|>\varepsilon\right\}}\left(q_{j}^{b}(t-s, x+w, z)-q_{j}^{b}(t-s, x, z)\right) \frac{\mathcal{A}(d,-\beta) b(x, w)}{|w|^{d+\beta}} d w\right) \\
& \times \mathcal{S}_{z}^{b} p_{0}(s, z, y) d z d s \\
= & \int_{0}^{t} \int_{\mathbb{R}^{d}} \mathcal{S}_{x}^{b} q_{j}^{b}(t-s, x, z) \mathcal{S}_{z}^{b} p_{0}(s, z, y) d z d s
\end{aligned}
$$

exists and (3.6) as well as (3.7) holds for $n=j+1$. (The same proof verifies (3.6) when $n=0$.) On the other hand, in view of (3.7) and (3.8) for $n=j$, we have by the Fubini theorem,

$$
\begin{aligned}
& q_{j+1}^{b}(t, x, y) \\
= & \int_{0}^{t} \int_{\mathbb{R}^{d}} q_{j}^{b}(s, x, z) \mathcal{S}_{z}^{b} p_{0}(t-s, z, y) d z d s \\
= & \int_{0}^{t} \int_{\mathbb{R}^{d}}\left(\int_{0}^{s} \int_{\mathbb{R}^{d}} p_{0}(r, x, w) \mathcal{S}_{w}^{b} q_{j-1}^{b}(s-r, w, z) d r d w\right) \mathcal{S}_{z}^{b} p_{0}(t-s, z, y) d z d s \\
= & \int_{0}^{t} \int_{\mathbb{R}^{d}} p_{0}(r, x, w)\left(\int_{r}^{t} \int_{\mathbb{R}^{d}} \mathcal{S}_{w}^{b} q_{j-1}^{b}(s-r, w, z) \mathcal{S}_{z}^{b} p_{0}(t-s, z, y) d s d z\right) d w d r \\
= & \int_{0}^{t} \int_{\mathbb{R}^{d}} p_{0}(r, x, w) \mathcal{S}_{w}^{b} q_{j}^{b}(t-r, w, y) d w d r .
\end{aligned}
$$


This verifies that (3.8) also holds for $n=j+1$. The lemma is now established by induction.

Recall that $M_{b, \lambda}=\operatorname{esssup}_{x, z \in \mathbb{R}^{d},|z|>\lambda}|b(x, z)|=\left\|b_{\lambda}(x, z)\right\|_{\infty}$.

Lemma 3.4. For each $\lambda>0$, there are positive constants $A_{0}=A_{0}(d, \alpha, \beta, \lambda)$ and $C_{13}=$ $C_{13}(d, \alpha, \beta, \lambda)$ so that if $\|b\|_{\infty} \leq A_{0}$, then for every integer $n \geq 0$,

$$
\left|q_{n+1}^{b}(t, x, y)\right| \leq C_{13} 2^{-n} p_{M_{b, \lambda}}(t, x, y) \quad \text { for } t \in(0,1] \text { and } x, y \in \mathbb{R}^{d},
$$

(3.5) holds and so $\mathcal{S}_{x}^{b} q_{n}^{b}(t, x, y)$ exists pointwise in the sense of (1.5) with

$$
\left|\mathcal{S}_{x}^{b} q_{n}^{b}(t, x, y)\right| \leq 2^{-n} f_{0}(t, x, y) \quad \text { for } t \in(0,1] \text { and } x, y \in \mathbb{R}^{d}
$$

and

$$
\sum_{n=0}^{\infty} q_{n}^{b}(t, x, y) \geq \frac{1}{2} p_{0}(t, x, y) \quad \text { for } t \in(0,1] \text { and }|x-y| \leq 3 t^{1 / \alpha} .
$$

Proof. We take a positive constant $A_{0}$ so that $A_{0} \leq 1 \wedge\left[2\left(C_{7}+C_{8}\right) C_{10}+2 C_{12}\right]^{-1}$. We have by Lemma 3.1 and Lemma 3.3 that for every $b$ with $\|b\|_{\infty} \leq A_{0}$,

$\left|q_{n+1}^{b}(t, x, y)\right| \leq C_{11} 2^{-n} g_{M_{b, \lambda} / A_{0}}(t, x, y) \leq C_{11} A_{0}^{-1} 2^{-n} g_{M_{b, \lambda}}(t, x, y) \quad$ and $\quad\left|\mathcal{S}_{x}^{b} q_{n}^{b}(t, x, y)\right| \leq 2^{-n} f_{0}(t, x, y)$

for every $t \in(0,1]$ and $x, y \in \mathbb{R}^{d}$. This together with (2.14) establishes (3.14) and (3.15).

On the other hand, by (2.12) , there exists $c=c(d, \alpha, \beta) \geq 1$ so that $g_{a}(t, x, y) \leq c p_{0}(t, x, y)$ for $a \in[0,1]$ and $|x-y| \leq 3 t^{1 / \alpha}$ and $t \in(0,1]$. Take $A_{0}$ small enough so that $A_{0} \leq 1 \wedge\left[2\left(C_{7}+\right.\right.$ $\left.\left.C_{8}\right) C_{10}+2 C_{12}\right]^{-1}$ and $\sum_{n=1}^{\infty}\left(A_{0}\left(C_{7}+C_{8}\right) C_{10}\right)^{n} \leq \frac{1}{2 c C_{11}}$. Then for every $b$ with $\|b\|_{\infty} \leq A_{0}$, we have by Lemma 3.1 for $|x-y| \leq 3 t^{1 / \alpha}$ and $t \in(0,1]$ that

$$
\sum_{n=1}^{\infty}\left|q^{b}\right|_{n}(t, x, y) \leq c C_{11} \sum_{n=1}^{\infty}\left(A_{0}\left(C_{7}+C_{8}\right) C_{10}\right)^{n} p_{0}(t, x, y) \leq \frac{1}{2} p_{0}(t, x, y) .
$$

Consequently, for $|x-y| \leq 3 t^{1 / \alpha}$ and $t \in(0,1]$,

$$
\sum_{n=0}^{\infty} q_{n}^{b}(t, x, y) \geq p_{0}(t, x, y)-\sum_{n=1}^{\infty}\left|q_{n}^{b}(t, x, y)\right| \geq \frac{1}{2} p_{0}(t, x, y) .
$$

We now extend the results in Lemma 3.4 to any bounded $b$ that satisfies condition (1.2). For $\lambda>0$, define

$$
b^{(\lambda)}(x, z)=\lambda^{\beta / \alpha-1} b\left(\lambda^{-1 / \alpha} x, \lambda^{-1 / \alpha} z\right) .
$$

For a function $f$ on $\mathbb{R}^{d}$, set

$$
f^{(\lambda)}(x):=f\left(\lambda^{-1 / \alpha} x\right) .
$$

By a change of variable, one has from (1.1) and (1.3) that

$$
\Delta^{\alpha / 2} f^{(\lambda)}(x)=\lambda^{-1}\left(\Delta^{\alpha / 2} f\right)\left(\lambda^{-1 / \alpha} x\right)
$$

and

$$
\mathcal{S}^{b^{(\lambda)}} f^{(\lambda)}(x)=\lambda^{-1}\left(\mathcal{S}^{b} f\right)\left(\lambda^{-1 / \alpha} x\right) .
$$

We remark here that condition (1.2) used in establishing (3.18). Note that the transition density function $p_{0}(t, x, y)$ of the symmetric $\alpha$-stable process has the following scaling property:

$$
p_{0}(t, x, y)=\lambda^{-d / \alpha} p_{0}\left(\lambda^{-1} t, \lambda^{-1 / \alpha} x, \lambda^{-1 / \alpha} y\right)
$$

Recall $q_{n}^{b}(t, x, y)$ is the function defined inductively by (3.3) with $q_{0}^{b}(t, x, y):=p_{0}(t, x, y)$. 
Lemma 3.5. Suppose that $b$ is a bounded function on $\mathbb{R}^{d} \times \mathbb{R}^{d}$ satisfying (1.2). For every $\lambda>0$ and for every integer $n \geq 0$ and $t>0$,

$$
q_{n}^{b^{(\lambda)}}(t, x, y)=\lambda^{-d / \alpha} q_{n}^{b}\left(\lambda^{-1} t, \lambda^{-1 / \alpha} x, \lambda^{-1 / \alpha} y\right), \quad x, y \in \mathbb{R}^{d}
$$

or, equivalently,

$$
q_{n}^{b}(t, x, y)=\lambda^{d / \alpha} q_{n}^{b^{(\lambda)}}\left(\lambda t, \lambda^{1 / \alpha} x, \lambda^{1 / \alpha} y\right), \quad x, y \in \mathbb{R}^{d} .
$$

Proof. We prove it by induction. Clearly in view of (3.19), (3.20) holds when $n=0$. Suppose that (3.20) holds for $n=j \geq 0$. Then by the definition (3.3), (3.18) and (3.19),

$$
\begin{aligned}
& q_{j+1}^{b^{(\lambda)}}(t, x, y)=\int_{0}^{t} \int_{\mathbb{R}^{d}} q_{j}^{b^{(\lambda)}}(t-s, x, z) \mathcal{S}_{z}^{b^{(\lambda)}} p_{0}(s, z, y) d z d s \\
= & \int_{0}^{t} \int_{\mathbb{R}^{d}} \lambda^{-d / \alpha} q_{j}^{b}\left(\lambda^{-1}(t-s), \lambda^{-1 / \alpha} x, \lambda^{-1 / \alpha} z\right) \lambda^{-d / \alpha-1}\left(\mathcal{S}_{z}^{b} p_{0}\left(\lambda^{-1} s, \cdot, \lambda^{-1 / \alpha} y\right)\right)\left(\lambda^{-1 / \alpha} z\right) d z d s \\
= & \lambda^{-d / \alpha} \int_{0}^{\lambda^{-1} t} \int_{\mathbb{R}^{d}} q_{j}^{b}\left(\lambda^{-1} t-r, \lambda^{-1 / \alpha} x, w\right)\left(\mathcal{S}_{w}^{b} p_{0}\left(r, \cdot \lambda^{-1 / \alpha} y\right)\right)(w) d w d r \\
= & \lambda^{-d / \alpha} q_{j+1}^{b}\left(\lambda^{-1} t, \lambda^{-1 / \alpha} x, \lambda^{-1 / \alpha} y\right) .
\end{aligned}
$$

This proves that (3.20) holds for $n=j+1$ and so, by induction, it holds for every $n \geq 0$.

Recall that $A_{0}$ is the positive constant in Lemma 3.4.

Theorem 3.6. For every $\lambda>0$ and $A>0$, there is a positive constant $C_{14}=C_{14}(d, \alpha, \beta, A, \lambda)>$ 0 so that for every bounded function $b$ with $\|b\|_{\infty} \leq A$, that satisfies condition (1.2) and $n \geq 0$,

$$
\left|q_{n}^{b}(t, x, y)\right| \leq C_{14} 2^{-n}\left(t^{-d / \alpha} \wedge\left(\frac{t}{|x-y|^{d+\alpha}}+\frac{M_{b, \lambda} t}{|x-y|^{d+\beta}}\right)\right)
$$

for every $0<t \leq 1 \wedge\left(A_{0} /\|b\|_{\infty}\right)^{\alpha /(\alpha-\beta)}$ and $x, y \in \mathbb{R}^{d}$, and

$$
\sum_{n=0}^{\infty} q_{n}^{b}(t, x, y) \geq \frac{1}{2} p_{0}(t, x, y) \quad \text { for } 0<t \leq 1 \wedge\left(A_{0} /\|b\|_{\infty}\right)^{\alpha /(\alpha-\beta)} \text { and }|x-y| \leq 3 t^{1 / \alpha} .
$$

Moreover, for every $n \geq 0$, (3.5) holds and so $\mathcal{S}_{x}^{b} q_{n}^{b}(t, x, y)$ exists pointwise in the sense of (1.5) with

$$
\left|\mathcal{S}_{x}^{b} q_{n}^{b}(t, x, y)\right| \leq 2^{-n}\left(\|b\|_{\infty} / A_{0}\right) f_{0}(t, x, y)
$$

for every $0<t \leq 1 \wedge\left(A_{0} /\|b\|_{\infty}\right)^{\alpha /(\alpha-\beta)}$ and $x, y \in \mathbb{R}^{d}$. Moreover, (3.6) and (3.8) hold.

Proof. In view of Lemma 3.4, it suffices to prove the theorem for $A_{0}<\|b\|_{\infty} \leq A$. Set $r=\left(\|b\|_{\infty} / A_{0}\right)^{\alpha /(\alpha-\beta)}$. The function $b^{(r)}$ defined by (3.17) has the property $\left\|b^{(r)}\right\|_{\infty}=A_{0}$. Thus by Lemma 3.4, there is a constant $C_{14}=C_{14}(d, \alpha, \beta, A, \lambda):=C_{13}\left(d, \alpha, \beta, r^{1 / \alpha} \lambda\right)>0$ so that for every integer $n \geq 0$,

$$
\left|q_{n}^{b^{(r)}}(t, x, y)\right| \leq C_{14} 2^{-n} p_{M_{b(r), r^{1 / \alpha_{\lambda}}}}(t, x, y) \quad \text { for } t \in(0,1] \text { and } x, y \in \mathbb{R}^{d},
$$

(3.5) holds and so $\mathcal{S}_{x}^{b^{(r)}} q_{n}^{b^{(r)}}(t, x, y)$ exists pointwise in the sense of (1.5) with

$$
\left|\mathcal{S}_{x}^{b^{(r)}} q_{n}^{b^{(r)}}(t, x, y)\right| \leq 2^{-n} f_{0}(t, x, y) \quad \text { for } t \in(0,1] \text { and } x, y \in \mathbb{R}^{d},
$$


and

$$
\sum_{n=0}^{\infty} q_{n}^{b^{(r)}}(t, x, y) \geq \frac{1}{2} p_{0}(t, x, y) \quad \text { for } t \in(0,1] \text { and }|x-y| \leq 3 t^{1 / \alpha} .
$$

Noting $r^{1-\beta / \alpha} M_{b^{(r)}, r^{1 / \alpha} \lambda}=M_{b, \lambda}$, we have by (3.21), (3.25) and (2.3) that for every $0<t \leq$ $1 / r=\left(A_{0} /\|b\|_{\infty}\right)^{\alpha /(\alpha-\beta)}$ and $x, y \in \mathbb{R}^{d}$

$$
\begin{aligned}
\left|q_{n}^{b}(t, x, y)\right| & =r^{d / \alpha}\left|q_{n}^{b^{(r)}}\left(r t, r^{1 / \alpha} x, r^{1 / \alpha} y\right)\right| \\
& \leq C_{14} 2^{-n} r^{d / \alpha} p_{M_{b^{(r),}, r^{1 / \alpha} \lambda}}\left(r t, r^{1 / \alpha} x, r^{1 / \alpha} y\right) \\
& \leq 2 C C_{14} 2^{-n}\left(t^{-d / \alpha} \wedge\left(\frac{t}{|x-y|^{d+\alpha}}+\frac{r^{1-\beta / \alpha} M_{b^{(r)}, r^{1 / \alpha} \lambda} t}{|x-y|^{d+\beta}}\right)\right) \\
& \leq 2 C C_{14} 2^{-n}\left(t^{-d / \alpha} \wedge\left(\frac{t}{|x-y|^{d+\alpha}}+\frac{M_{b, \lambda} t}{|x-y|^{d+\beta}}\right)\right),
\end{aligned}
$$

which establishes (3.22). Similarly, (3.23) follows from (3.19), and (3.27), while the conclusion of (3.24) is a direct consequence of (3.18), (3.21) and (3.26). That (3.6) and (3.8) hold follows directly from Lemma 3.3 and Lemma 3.5 .

Recall that $q^{b}(t, x, y):=\sum_{n=0}^{\infty} q_{n}^{b}(t, x, y)$, whenever it is convergent. The following theorem follows immediately from Lemmas 3.2, 3.4 and Theorem 3.6.

Theorem 3.7. For every $\lambda>0$ and $A>0$, let $C_{14}=C_{14}(d, \alpha, \beta, A, \lambda)$ be the constant in Theorem [3.6. Then for every bounded function $b$ with $\|b\|_{\infty} \leq A$ that satisfies condition (1.2), $q^{b}(t, x, y)$ is well defined and is jointly continuous in $\left(0,1 \wedge\left(A_{0} /\|b\|_{\infty}\right)^{\alpha /(\alpha-\beta)}\right] \times \mathbb{R}^{d} \times \mathbb{R}^{d}$. Moreover,

$$
\left|q^{b}(t, x, y)\right| \leq 2 C_{14}\left(t^{-d / \alpha} \wedge\left(\frac{t}{|x-y|^{d+\alpha}}+\frac{M_{b, \lambda} t}{|x-y|^{d+\beta}}\right)\right)
$$

and $\mathcal{S}_{x}^{b} q^{b}(t, x, y)$ exists pointwise in the sense of (1.5) with

$$
\left|\mathcal{S}_{x}^{b} q^{b}(t, x, y)\right| \leq 2\left(\|b\|_{\infty} / A_{0}\right) f_{0}(t, x, y)
$$

for every $0<t \leq 1 \wedge\left(A_{0} /\|b\|_{\infty}\right)^{\alpha /(\alpha-\beta)}$ and $x, y \in \mathbb{R}^{d}$, and

$$
q^{b}(t, x, y) \geq \frac{1}{2} p_{0}(t, x, y) \quad \text { for } 0<t \leq 1 \wedge\left(A_{0} /\|b\|_{\infty}\right)^{\alpha /(\alpha-\beta)} \text { and }|x-y| \leq 3 t^{1 / \alpha} .
$$

Moreover, for every $0<t \leq 1 \wedge\left(A_{0} /\|b\|_{\infty}\right)^{\alpha /(\alpha-\beta)}$ and $x, y \in \mathbb{R}^{d}$,

$$
\begin{aligned}
q^{b}(t, x, y) & =p_{0}(t, x, y)+\int_{0}^{t} \int_{\mathbb{R}^{d}} q^{b}(t-s, x, z) \mathcal{S}_{z}^{b} p_{0}(s, z, y) d z d s \\
& =p_{0}(t, x, y)+\int_{0}^{t} \int_{\mathbb{R}^{d}} p_{0}(t-s, x, z) \mathcal{S}_{z}^{b} q^{b}(s, z, y) d z d s
\end{aligned}
$$

Theorem 3.8. Suppose that $b$ is a bounded function on $\mathbb{R}^{d} \times \mathbb{R}^{d}$ satisfying (1.2). Let $A_{0}$ be the constant in Lemma 3.4. Then for every $t, s>0$ with $t+s \leq 1 \wedge\left(A_{0} /\|b\|_{\infty}\right)^{\alpha /(\alpha-\beta)}$ and $x, y \in \mathbb{R}^{d}$,

$$
\int_{\mathbb{R}^{d}} q^{b}(t, x, z) q^{b}(s, z, y) d z=q^{b}(t+s, x, y) .
$$


Proof. In view of Theorem 3.6, we have

$$
\int_{\mathbb{R}^{d}} q^{b}(t, x, z) q^{b}(s, z, y) d z=\sum_{j=0}^{\infty} \sum_{n=0}^{j} \int_{\mathbb{R}^{d}} q_{n}^{b}(t, x, z) q_{j-n}^{b}(s, z, y) d z .
$$

So it suffices to show that for every $j \geq 0$,

$$
\sum_{n=0}^{j} \int_{\mathbb{R}^{d}} q_{n}^{b}(t, x, z) q_{j-n}^{b}(s, z, y) d z=q_{j}^{b}(t+s, x, y)
$$

Clearly, (3.32) holds for $j=0$. Suppose that (3.32) holds for $j=l \geq 1$. Then we have by Fubini's theorem and the estimates in (3.1) and Theorem 3.6 .

$$
\begin{aligned}
& \sum_{n=0}^{l+1} \int_{\mathbb{R}^{d}} q_{n}^{b}(t, x, z) q_{l+1-n}^{b}(s, z, y) d z \\
= & \int_{\mathbb{R}^{d}} q_{l+1}^{b}(t, x, z) p_{0}(s, z, y) d z+\sum_{n=0}^{l} \int_{\mathbb{R}^{d}} q_{n}^{b}(t, x, z) q_{l+1-n}^{b}(s, z, y) d z \\
= & \int_{\mathbb{R}^{d}}\left(\int_{0}^{t} \int_{\mathbb{R}^{d}} q_{l}^{b}(t-r, x, w) \mathcal{S}_{w}^{b} p_{0}(r, w, z) d w d r\right) p_{0}(s, z, y) d z \\
& +\sum_{n=0}^{l} \int_{\mathbb{R}^{d}} q_{n}^{b}(t, x, z)\left(\int_{0}^{s} \int_{\mathbb{R}^{d}} q_{l-n}^{b}(s-r, z, w) \mathcal{S}_{w}^{b} p_{0}(r, w, y) d w d r\right) d z \\
= & \int_{0}^{t} \int_{\mathbb{R}^{d}} q_{l}^{b}(t-r, x, w) \mathcal{S}_{w}^{b} p_{0}(r+s, w, y) d w d r \\
& +\int_{0}^{s} \int_{\mathbb{R}^{d}} q_{l}^{b}(t+s-r, x, w) \mathcal{S}_{w}^{b} p_{0}(r, w, y) d w d r \\
= & q_{l+1}^{b}(t+s, x, y) .
\end{aligned}
$$

This proves that (3.32) holds for $j=l+1$. So by induction, we conclude that (3.32) holds for every $j \geq 0$.

For notational simplicity, denote $1 \wedge\left(A_{0} /\|b\|_{\infty}\right)^{\alpha /(\alpha-\beta)}$ by $\delta_{0}$. In view of Theorem 3.8, we can uniquely extend the definition of $q^{b}(t, x, y)$ to $t>\delta_{0}$ by using the Chapman-Kolmogorov equation recursively as follows.

Suppose that $q^{b}(t, x, y)$ has been defined and satisfies the Chapman-Kolmogorov equation (3.31) on $\left(0, k \delta_{0}\right] \times \mathbb{R}^{d} \times \mathbb{R}^{d}$. Then for $t \in\left(k \delta_{0},(k+1) \delta_{0}\right]$, define

$$
q^{b}(t, x, y)=\int_{\mathbb{R}^{d}} q^{b}(s, x, z) q^{b}(r, z, y) d z, \quad x, y \in \mathbb{R}^{d}
$$

for any $s, r \in\left(0, k \delta_{0}\right]$ so that $s+r=t$. Such $q^{b}(t, x, y)$ is well defined on $(0, \infty) \times \mathbb{R}^{d} \times \mathbb{R}^{d}$ and satisfies (3.31) for every $s, t>0$. Moreover, since Chapman-Kolmogorov equation holds for $q^{b}(t, x, y)$ for all $t, s>0$, we have by Theorem 3.7 and (2.3)-(2.4) that for every $A \geq A_{0}$, there are constants $c_{i}=c_{i}(d, \alpha, \beta, A, \lambda), i=1,2$, so that for every $b(x, z)$ satisfying (1.2) with $\|b\|_{\infty} \leq A$,

$$
\left|q^{b}(t, x, y)\right| \leq c_{1} e^{c_{2} t} p_{M_{b, \lambda}}(t, x, y) \quad \text { for every } t>0 \text { and } x, y \in \mathbb{R}^{d}
$$


Theorem 3.9. $q^{b}(t, x, y)$ satisfies (3.29) and (3.30) for every $t>0$ and $x, y \in \mathbb{R}^{d}$.

Proof. Let $\delta_{0}:=1 \wedge\left(A_{0} /\|b\|_{\infty}\right)^{\alpha /(\alpha-\beta)}$. It suffices to prove that for every $n \geq 1$, (3.29) and (3.30) hold for all $t \in\left(0, n \delta_{0}\right]$ and $x, y \in \mathbb{R}^{d}$.

Clearly, (3.29) holds for $t \in\left(0, n \delta_{0}\right]$ with $n=1$. Suppose that (3.29) holds for $t \in\left(0, n \delta_{0}\right]$ with $n=k$. For $t \in\left(k \delta_{0},(k+1) \delta_{0}\right]$, take $l, s \in\left(0, k \delta_{0}\right]$ so that $l+s=t$. Then we have by Fubini's theorem, Chapman-Kolmogorov equation of $q^{b}$, Lemma 2.5, (3.1) and (3.34),

$$
\begin{aligned}
q^{b}(l+s, x, y)= & \int_{\mathbb{R}^{d}} q^{b}(l, x, z) q^{b}(s, z, y) d z \\
= & \int_{\mathbb{R}^{d}} q^{b}(l, x, z)\left(p_{0}(s, z, y)+\int_{0}^{s} \int_{\mathbb{R}^{d}} q^{b}(s-r, z, \omega) \mathcal{S}_{\omega}^{b} p_{0}(r, \omega, y) d \omega d r\right) d z \\
= & \int_{\mathbb{R}^{d}} p_{0}(l, x, z) p_{0}(s, z, y) d z \\
& +\int_{\mathbb{R}^{d}}\left(\int_{0}^{l} \int_{\mathbb{R}^{d}} q^{b}(l-u, x, \eta) \mathcal{S}_{\omega}^{b} p_{0}(u, \eta, z) d \eta d u\right) p_{0}(s, z, y) d z \\
& +\int_{0}^{s} \int_{\mathbb{R}^{d}} q^{b}(l+s-r, x, \omega) \mathcal{S}_{\omega}^{b} p_{0}(r, \omega, y) d \omega d r \\
= & p_{0}(l+s, x, y)+\int_{0}^{l} \int_{\mathbb{R}^{d}} q^{b}(l-u, x, \eta) \mathcal{S}_{\omega}^{b} p_{0}(u+s, \eta, y) d \eta d u \\
& +\int_{0}^{s} \int_{\mathbb{R}^{d}} q^{b}(l+s-r, x, \omega) \mathcal{S}_{\omega}^{b} p_{0}(r, \omega, y) d \omega d r \\
= & p_{0}(l+s, x, y)+\int_{0}^{l+s} \int_{\mathbb{R}^{d}} q^{b}(l+s-r, x, z) \mathcal{S}_{z}^{b} p_{0}(r, z, y) d z d r .
\end{aligned}
$$

By the similar procedure as above, we can also prove that (3.30) holds for every $t>0$ and $x, y \in \mathbb{R}^{d}$.

Theorem 3.10. Suppose that $b$ is a bounded function on $\mathbb{R}^{d} \times \mathbb{R}^{d}$ satisfying (1.2). Then $q^{b}(t, x, y)$ is the unique continuous kernel that satisfies the Chapman-Kolmogorov equation (3.31) on $(0, \infty) \times \mathbb{R}^{d} \times \mathbb{R}^{d}$ and that for some $\varepsilon>0$,

$$
\left|q^{b}(t, x, y)\right| \leq c p_{1}(t, x, y)
$$

and (3.29) hold for $(t, x, y) \in(0, \varepsilon] \times \mathbb{R}^{d} \times \mathbb{R}^{d}$. Moreover, (3.34) holds for $q^{b}(t, x, y)$.

Proof. Suppose that $\bar{q}$ is any continuous kernel that satisfies, for some $\varepsilon>0$, (3.29) and (3.35) hold for $(t, x, y) \in(0, \varepsilon] \times \mathbb{R}^{d} \times \mathbb{R}^{d}$. Without loss of generality, we may and do assume that $\varepsilon<1 \wedge\left(A_{0} /\|b\|_{\infty}\right)^{\alpha /(\alpha-\beta)}$. Using (3.29) recursively, one gets

$$
\bar{q}(t, x, y)=\sum_{j=0}^{n} q_{j}^{b}(t, x, y)+\int_{0}^{t} \int_{\mathbb{R}^{d}} \bar{q}(t-s, x, z)\left(\mathcal{S}^{b} p_{0}\right)_{z}^{*, n+1}(s, z, y) d s d z .
$$

Here $\left(\mathcal{S}^{b} p_{0}\right)_{z}^{*, n}(s, z, y)$ denotes the $n$th convolution operation of the function $\mathcal{S}_{z}^{b} p_{0}(s, z, y)$; that is, $\left(\mathcal{S}^{b} p_{0}\right)_{z}^{*, 1}(s, z, y)=\mathcal{S}_{z}^{b} p_{0}(s, z, y)$ and

$$
\left(\mathcal{S}^{b} p_{0}\right)_{z}^{*, n}(s, z, y)=\int_{0}^{s} \int_{\mathbb{R}^{d}} \mathcal{S}_{z}^{b} p_{0}(r, z, w)\left(\mathcal{S}^{b} p_{0}\right)_{w}^{*, n-1}(s-r, w, y) d w d r \quad \text { for } n \geq 2 .
$$


It follows from (3.6) that

$$
\mathcal{S}_{z}^{b} q_{n}^{b}(s, z, y)=\left(\mathcal{S}^{b} p_{0}\right)_{z}^{*, n+1}(s, z, y)
$$

Thus, by (3.36) we have

$$
\bar{q}(t, x, y)=\sum_{j=0}^{n} q_{j}^{b}(t, x, y)+\int_{0}^{t} \int_{\mathbb{R}^{d}} \bar{q}(t-s, x, z) \mathcal{S}_{z}^{b} q_{n}^{b}(s, z, y) d s d z .
$$

By the condition (3.35) and (3.24), there is a constant $c_{1}>0$ so that for every $n \geq 1$,

$$
\left|\int_{0}^{t} \int_{\mathbb{R}^{d}} \bar{q}(t-s, x, z)\left(\mathcal{S}^{b} p_{0}\right)_{z}^{*, n}(s, z, y) d s d z\right| \leq c_{1} 2^{-n} \int_{0}^{t} \int_{\mathbb{R}^{d}} p_{1}(t-s, x, z) f_{0}(s, z, y) d s d z .
$$

Noting that $p_{1}(t, x, y) \asymp g_{1}(t, x, y)$ on $(0,1] \times \mathbb{R}^{d} \times \mathbb{R}^{d}$ and

$$
\begin{aligned}
\int_{0}^{t} \int_{\mathbb{R}^{d}} f_{0}(s, z, y) d z d s & \leq \int_{0}^{t} \int_{|y-z| \leq s^{1 / \alpha}} s^{-(d+\beta) / \alpha} d z d s+\int_{0}^{t} \int_{|y-z|>s^{1 / \alpha}} \frac{1}{|y-z|^{d+\beta}} d z d s \\
& =c_{2} t^{1-\beta / \alpha} .
\end{aligned}
$$

Then by the similar proof in Lemma 2.5, we can get

$$
\int_{0}^{t} \int_{\mathbb{R}^{d}} p_{1}(t-s, x, z) f_{0}(s, z, y) d s d z \leq c_{3} p_{1}(t, x, y) .
$$

It follows that

$$
\bar{q}(t, x, y)=\sum_{n=0}^{\infty} q_{n}^{b}(t, x, y)=q^{b}(t, x, y)
$$

for every $t \in(0, \varepsilon]$ and $x, y \in \mathbb{R}^{d}$. Since both $\bar{q}$ and $q^{b}$ satisfy the Chapman-Kolmogorov equation (3.31), $\bar{q}=q^{b}$ on $(0, \infty) \times \mathbb{R}^{d} \times \mathbb{R}^{d}$.

Remark 3.11. It follows from the definition of $q_{n}^{b}(t, x, y)$ and Lemma 3.3 that $\left(\mathcal{S}^{b} p_{0}\right)^{*, n+1}(s, z, y)=$ $\mathcal{S}_{z}^{b} q_{n}^{b}(s, z, y)$.

In view of Lemma 3.5 and Chapman-Kolmogorov equation, we have

Theorem 3.12. Suppose that $b$ is a bounded function on $\mathbb{R}^{d} \times \mathbb{R}^{d}$ satisfying (1.2). $q^{b}(t, x, y)=$ $\lambda^{d / \alpha} q^{b^{(\lambda)}}\left(\lambda t, \lambda^{1 / \alpha} x, \lambda^{1 / \alpha} y\right)$ on $(0, \infty) \times \mathbb{R}^{d} \times \mathbb{R}^{d}$, where $b^{(\lambda)}(x, z):=\lambda^{\beta / \alpha-1} b\left(\lambda^{-1 / \alpha} x, \lambda^{-1 / \alpha} z\right)$.

For a bounded function $f$ on $\mathbb{R}^{d}, t>0$ and $x \in \mathbb{R}^{d}$, we define

$$
T_{t}^{b} f(x)=\int_{\mathbb{R}^{d}} q^{b}(t, x, y) f(y) d y \quad \text { and } \quad P_{t} f(x)=\int_{\mathbb{R}^{d}} p_{0}(t, x, y) f(y) d y .
$$

The following lemma follows immediately from (3.31) and (3.33).

Lemma 3.13. Suppose that $b$ is a bounded function on $\mathbb{R}^{d} \times \mathbb{R}^{d}$ satisfying (1.2). For all $s, t>0$, we have $T_{t+s}^{b}=T_{t}^{b} T_{s}^{b}$. 
Theorem 3.14. Let $b$ be a bounded function on $\mathbb{R}^{d} \times \mathbb{R}^{d}$ satisfying (1.2). Then for every $f \in C_{b}^{2}\left(\mathbb{R}^{d}\right)$,

$$
T_{t}^{b} f(x)-f(x)=\int_{0}^{t} T_{s}^{b} \mathcal{L}^{b} f(x) d s \quad \text { for every } t>0, x \in \mathbb{R}^{d} .
$$

Proof. Note that by Theorem 3.9, for each bounded Borel function $f$ in $\mathbb{R}^{d}$,

$$
T_{t}^{b} f(x)=P_{t} f(x)+\int_{0}^{t} T_{t-s}^{b} \mathcal{S}^{b} P_{s} f(x) d s=P_{t} f(x)+\int_{0}^{t} T_{s}^{b} \mathcal{S}^{b} P_{t-s} f(x) d s .
$$

Hence, for $f \in C_{b}^{2}\left(\mathbb{R}^{d}\right)$,

$$
\begin{aligned}
& T_{t}^{b} f(x)-f(x) \\
= & P_{t} f(x)-f(x)+\int_{0}^{t} T_{s}^{b} \mathcal{S}^{b} f(x) d s+\int_{0}^{t} T_{s}^{b} \mathcal{S}^{b}\left(P_{t-s} f-f\right)(x) d s \\
= & \int_{0}^{t} P_{s} \Delta^{\alpha / 2} f(x) d s+\int_{0}^{t} T_{s}^{b} \mathcal{S}^{b} f(x) d s+\int_{0}^{t} T_{s}^{b} \mathcal{S}^{b}\left(P_{t-s} f-f\right)(x) d s \\
= & \int_{0}^{t} T_{s}^{b} \Delta^{\alpha / 2} f(x) d s-\int_{0}^{t}\left(\int_{0}^{s} T_{r}^{b} \mathcal{S}^{b} P_{s-r}\left(\Delta^{\alpha / 2} f\right)(x) d r\right) d s \\
& +\int_{0}^{t} T_{s}^{b} \mathcal{S}^{b} f(x) d s+\int_{0}^{t} T_{s}^{b} \mathcal{S}^{b}\left(P_{t-s} f-f\right)(x) d s \\
= & \int_{0}^{t} T_{s}^{b}\left(\Delta^{\alpha / 2}+\mathcal{S}^{b}\right) f(x) d s-\int_{0}^{t}\left(\int_{r}^{t} T_{r}^{b} \mathcal{S}^{b} P_{s-r}\left(\Delta^{\alpha / 2} f\right)(x) d s\right) d r \\
& +\int_{0}^{t} T_{s}^{b} \mathcal{S}^{b}\left(P_{t-s} f-f\right)(x) d s \\
= & \int_{0}^{t} T_{s}^{b} \mathcal{L}^{b} f(x) d s-\int_{0}^{t} T_{r}^{b} \mathcal{S}^{b}\left(P_{t-r} f-f\right)(x) d r+\int_{0}^{t} T_{s}^{b} \mathcal{S}^{b}\left(P_{t-s} f-f\right)(x) d s \\
= & \int_{0}^{t} T_{s}^{b} \mathcal{L}^{b} f(x) d s .
\end{aligned}
$$

Here in the third inequality, we used (3.40); while in the fifth inequality we used Lemma2.2 and (3.34), which allow the interchange of the integral $\operatorname{sign} \int_{r}^{t}$ with $T_{r}^{b} \mathcal{S}^{b}$, and the fact that

$$
\int_{r}^{t} P_{s-r}\left(\Delta^{\alpha / 2} f\right)(x) d s=\int_{r}^{t}\left(\frac{d}{d s} P_{s-r} f(x)\right) d s=P_{t-r} f(x)-f(x) .
$$

Theorem 3.15. Let $b$ be a bounded function on $\mathbb{R}^{d} \times \mathbb{R}^{d}$ satisfying (1.2). Then $q^{b}(t, x, y)$ is jointly continuous in $(0, \infty) \times \mathbb{R}^{d} \times \mathbb{R}^{d}$ and $\int_{\mathbb{R}^{d}} q^{b}(t, x, y) d y=1$ for every $x \in \mathbb{R}^{d}$ and $t>0$.

Proof. By Lemma 3.13, we have

$$
q^{b}(t+s, x, y)=\int_{\mathbb{R}^{d}} q^{b}(t, x, z) q^{b}(s, z, y) d z, \quad x, y \in \mathbb{R}^{d}, s, t>0
$$


Continuity of $q^{b}(t, x, y)$ in $(t, x, y) \in(0, \infty) \times \mathbb{R}^{d} \times \mathbb{R}^{d}$ follows from Theorem 3.7, (3.41) and the dominated convergence theorem. For $n \geq 1$ and $t \in(0, T]$, it follows from (3.1), Lemma [2.5. Theorem 3.6 and Fubini's Theorem that for every $t \in\left(0,1 \wedge\left(A_{0} /\|b\|_{\infty}\right)^{\alpha /(\alpha-\beta)}\right]$,

$$
\begin{aligned}
\int_{\mathbb{R}^{d}} q_{n}^{b}(t, x, y) d y & =\int_{\mathbb{R}^{d}} \int_{\mathbb{R}^{d}} \int_{0}^{t} q_{n-1}^{b}(t-s, x, z) \mathcal{S}_{z}^{b} p_{0}(s, z, y) d s d z d y \\
& =\int_{\mathbb{R}^{d}} \int_{0}^{t} q_{n-1}^{b}(t-s, x, z) \mathcal{S}_{z}^{b}\left(\int_{\mathbb{R}^{d}} p_{0}(s, z, y) d y\right) d s d z=0 .
\end{aligned}
$$

Hence we have by Lemma 3.4.

$$
\int_{\mathbb{R}^{d}} q^{b}(t, x, y) d y=\int_{\mathbb{R}^{d}} p_{0}(t, x, y) d y=1
$$

for $t \in\left(0,1 \wedge\left(A_{0} /\|b\|_{\infty}\right)^{\alpha /(\alpha-\beta)}\right]$. This conservativeness property extends to all $t>0$ by (3.41).

Theorem 1.1 now follows from (2.3)-(2.4), Theorems 3.7, 3.9, 3.10, 3.14 and 3.15,

\section{$4 C_{\infty}$-Semigroups and Positivity}

Recall that $A_{0}$ is the positive constant in Lemma 3.4 .

Lemma 4.1. Suppose that $b$ is a bounded function on $\mathbb{R}^{d} \times \mathbb{R}^{d}$ satisfying condition (1.2). Then $\left\{T_{t}^{b}, t>0\right\}$ is a strongly continuous semigroup in $C_{\infty}\left(\mathbb{R}^{d}\right)$.

Proof. The following proof is a minor modification of that for [9, Proposition 2.3]. For reader's convenience, we spell out the details. Since $q^{b}(t, x, y)$ is continuous by Theorem 3.15, it follows that $T_{t}^{b}$ maps bounded continuous functions to continuous function for every $t>0$. Moreover, by (3.34) and the semigroup property of $q^{b}(t, x, y)$, there are constants $c_{1}$ and $c_{2}$ so that

$$
\left|q^{b}(t, x, y)\right| \leq c_{1} e^{c_{2} t} p_{M_{b, \lambda}}(t, x, y) \quad \text { for every } t>0 \text { and } x, y \in \mathbb{R}^{d} .
$$

Thus, for every $f \in C_{\infty}\left(\mathbb{R}^{d}\right)$ and $t>0$,

$$
\lim _{x \rightarrow \infty}\left|T_{t}^{b} f(x)\right| \leq \lim _{x \rightarrow \infty} c_{1} e^{c_{2} t} \int_{\mathbb{R}^{d}} p_{M_{b, \lambda}}(t, x, y)|f(y)| d y=0
$$

and so $T_{t}^{b} f \in C_{\infty}\left(\mathbb{R}^{d}\right)$. On the other hand, given $f \in C_{\infty}\left(\mathbb{R}^{d}\right)$, for every $\varepsilon>0$, there is $\delta>0$ so that $|f(x)-f(y)| \leq \varepsilon$ whenever $|x-y| \leq \delta$. Since

$$
\lim _{t_{0} \rightarrow 0} \sup _{t \leq t_{0}} \sup _{x \in \mathbb{R}^{d}} \int_{|y-x| \geq \delta}\left|q^{b}(t, x, y)\right| d y \leq \lim _{t_{0} \rightarrow 0} c e^{c_{2} t_{0}} \sup _{t \leq t_{0}} \sup _{x \in \mathbb{R}^{d}} \int_{|y-x| \geq \delta} p_{M_{b, \lambda}}(t, x, y) d y=0,
$$

we have

$$
\begin{aligned}
& \lim _{t \rightarrow 0} \sup _{x \in \mathbb{R}^{d}}\left|T_{t}^{b} f(x)-f(x)\right| \\
= & \lim _{t \rightarrow 0} \sup _{x \in \mathbb{R}^{d}}\left|\int_{\mathbb{R}^{d}} q^{b}(t, x, y)(f(y)-f(x)) d y\right| \\
\leq & \lim _{t \rightarrow 0} \sup _{x \in \mathbb{R}^{d}} \int_{|y-x|<\delta}\left|q^{b}(t, x, y)\right||f(y)-f(x)| d y+2\|f\|_{\infty} \lim _{t \rightarrow 0} \sup _{x \in \mathbb{R}^{d}} \int_{|y-x| \geq \delta}\left|q^{b}(t, x, y)\right| d y \\
\leq & \varepsilon \lim _{t \rightarrow 0} \sup _{x \in \mathbb{R}^{d}} \int_{\mathbb{R}^{d}} c_{1} e^{c_{2} t} p_{1}(t, x, y) d y=c_{1} \varepsilon .
\end{aligned}
$$


Thus $\lim _{t \rightarrow 0} \sup _{x \in \mathbb{R}^{d}}\left|T_{t}^{b} f(x)-f(x)\right|=0$. This proves that $T_{t}^{b}$ is a strongly continuous semigroup in $C_{\infty}\left(\mathbb{R}^{d}\right)$.

Lemma 4.2. Let $b$ be a bounded function on $\mathbb{R}^{d} \times \mathbb{R}^{d}$ satisfying (1.18). For each $f \in C_{\infty}^{2}\left(\mathbb{R}^{d}\right)$, $\mathcal{L}^{b} f(x)$ exists pointwise and is in $C_{\infty}\left(\mathbb{R}^{d}\right)$.

Proof. Suppose that $\gamma \in(0,2)$ and $f \in C_{\infty}^{2}\left(\mathbb{R}^{d}\right)$. Denote $\sum_{i, j=1}^{d}\left|\partial_{i j}^{2} f(x)\right|$ by $\left|D^{2} f(x)\right|$. Let $R>1$ to be chosen later. Then for each $x \in \mathbb{R}^{d}$, we have by Taylor expansion,

$$
\begin{aligned}
\Phi_{f}(x):= & \int_{\mathbb{R}^{d}}\left|f(x+z)-f(x)-\nabla f(x) \cdot z \mathbb{1}_{\{|z| \leq 1\}}\right| \frac{1}{|z|^{d+\gamma}} d z \\
\leq & \int_{|z| \leq 1}\left|f(x+z)-f(x)-\nabla f(x) \cdot z \mathbb{1}_{\{|z| \leq 1\} \mid}\right| \frac{1}{|z|^{d+\gamma}} d z \\
& +\int_{1<|z| \leq R}|f(x+z)-f(x)| \frac{1}{|z|^{d+\gamma}} d z+\int_{|z|>R}|f(x+z)-f(x)| \frac{1}{|z|^{d+\gamma}} d z \\
\leq & \sup _{|y| \leq 1}\left|D^{2} f(x+y)\right| \int_{|z| \leq 1}|z|^{2-d-\gamma} d z+\int_{1<|z| \leq R}|f(x+z)-f(x)| \frac{1}{|z|^{d+\gamma}} d z \\
& +2\left\|\left.\left|f \|_{\infty} \int_{|z|>R}\right| z\right|^{-d-\gamma} d z\right. \\
= & c \sup _{|y| \leq 1}\left|D^{2} f(x+y)\right|+\int_{1<|z| \leq R}|f(x+z)-f(x)| \frac{1}{|z|^{d+\gamma}} d z+c R^{-\gamma}\|f\|_{\infty} .
\end{aligned}
$$

For any given $\varepsilon>0$, we can take $R$ large so that $c R^{-\gamma}\|f\|_{\infty}<\varepsilon / 2$ to conclude that

$$
\lim _{|x| \rightarrow \infty} \int_{\mathbb{R}^{d}}\left|f(x+z)-f(x)-\nabla f(x) \cdot z \mathbb{1}_{\{|z| \leq 1\}}\right| \frac{1}{|z|^{d+\gamma}} d z=0 .
$$

By the same reason, applying the above argument to function $x \mapsto f(x+y)-f(x)$ in place of $f$ yields that for every $\varepsilon>0$ and $x_{0} \in \mathbb{R}^{d}$, there is $\delta>0$ so that

$$
\Phi_{f(\cdot+y)-f}\left(x_{0}\right)<\varepsilon \quad \text { for every }|y|<\delta .
$$

It follows from the last two displays, the definition of $\mathcal{L}^{b}$ and (1.4) that $\mathcal{L}^{b} f(x)$ exists for every $x \in \mathbb{R}^{d}$ and $\mathcal{L}^{b} f \in C_{\infty}\left(\mathbb{R}^{d}\right)$.

Proof of Theorem 1.2. Since $b$ satisfies condition (1.18), then $\mathcal{L}^{b} f \in C_{\infty}\left(\mathbb{R}^{d}\right)$ for every $f \in C_{c}^{2}\left(\mathbb{R}^{d}\right)$ by Lemma 4.2, Let $\widehat{\mathcal{L}}^{b}$ denote the infinitesimal generator of the strongly continuous semigroup $\left\{T_{t}^{b} ; t \geq 0\right\}$ in $C_{\infty}\left(\mathbb{R}^{d}\right)$, which is a closed linear operator. It follows from Theorem 3.14. Lemmas 4.1 and 4.2 that for every $f \in C_{\infty}^{2}\left(\mathbb{R}^{d}\right),\left(T_{t}^{b} f(x)-f(x)\right) / t$ converges uniformly to $\mathcal{L}^{b} f(x)$ as $t \rightarrow 0$. So

$$
C_{\infty}^{2}\left(\mathbb{R}^{d}\right) \subset D\left(\widehat{\mathcal{L}}^{b}\right) \quad \text { and } \quad \widehat{\mathcal{L}}^{b} f=\mathcal{L}^{b} f \quad \text { for } f \in C_{\infty}^{2}\left(\mathbb{R}^{d}\right) .
$$

In view of Theorem 3.7, there are constants $c_{1}, c_{2}>0$ so that (4.1) holds. This implies that

$$
\sup _{x \in \mathbb{R}^{d}} \int_{0}^{\infty} e^{-\lambda t}\left|T_{t}^{b} f\right|(x) d t \leq c_{\lambda}\|f\|_{\infty}, \quad f \in C_{\infty}\left(\mathbb{R}^{d}\right),
$$


for every $\lambda>c_{2}$. Observe that $e^{-c_{2} t} T_{t}^{b}$ is a strongly continuous semigroup in $C_{\infty}\left(\mathbb{R}^{d}\right)$ whose infinitesimal generator is $\widehat{\mathcal{L}}^{b}-c_{2}$. The above display implies that $(0, \infty)$ is contained in the residual set $\rho\left(\widehat{\mathcal{L}}^{b}-c_{2}\right)$ of $\widehat{\mathcal{L}}^{b}-c_{2}$. Therefore by Theorem 3.15 and the Hille-Yosida-Ray theorem [16, p165], $\left\{e^{-c_{2} t} T_{t}^{b} ; t \geq 0\right\}$ is a positive preserving semigroup on $C_{\infty}\left(\mathbb{R}^{d}\right)$ if and only if $\widehat{\mathcal{L}}^{b}-c_{2}$ satisfies the positive maximum principle. On the other hand, Courrége's first theorem (see [1, p158]) tells us that $\widehat{\mathcal{L}}^{b}-c_{2}$ satisfies the positive maximum principle if and only if for each $x \in \mathbb{R}^{d}$,

$$
\frac{\mathcal{A}(d,-\alpha)}{|z|^{d+\alpha}}+\frac{\mathcal{A}(d,-\beta) b(x, z)}{|z|^{d+\beta}} \geq 0 \quad \text { for a.e. } z \in \mathbb{R}^{d} .
$$

Since $e^{-c_{2} t} T_{t}^{b}$ has a continuous integral kernel $e^{-c_{2} t} q^{b}(t, x, y)$, it follows that $q^{b}(t, x, y) \geq 0$ on $(0, \infty) \times \mathbb{R}^{d} \times \mathbb{R}^{d}$ if and only if for each $x \in \mathbb{R}^{d}$, (1.19) holds. If $b(x, z)=b(x)$ is a function of $x$ only, then by taking $|z| \rightarrow \infty$, one concludes that (1.19) holds if and only if $b(x) \geq 0$ on $\mathbb{R}^{d}$.

\section{$5 \quad$ Feller process and heat kernel estimates}

Throughout this section, $b$ is a bounded function satisfying condition (1.2) and (1.19). We will show that $q^{b}(t, x, y)>0$ and so it generates a Feller process $X^{b}$ that has strong Feller property. We further derive the upper and lower bound estimates on $q^{b}(t, x, y)$. We will first establish the Feller process $X^{b}$ and its connection to the martingale problem for $\left(\mathcal{L}^{b}, \mathcal{S}\left(\mathbb{R}^{d}\right)\right)$ under an additional assumption (1.18). We will then remove this additional assumption using an approximation method and the uniqueness result on $q^{b}(t, x, y)$ from Theorem 3.10 .

Suppose that $b$ is a bounded function satisfying conditions (1.2), (1.18) and (1.19). Then it follows from Theorem 1.2, Theorem 3.15, Lemma4.1 and Theorem 3.8, $T^{b}$ is a Feller semigroup. So it uniquely determines a conservative Feller process $X^{b}=\left\{X_{t}^{b}, t \geq 0, \mathbb{P}_{x}, x \in \mathbb{R}^{d}\right\}$ having $q^{b}(t, x, y)$ as its transition density function. Since, by Theorem 3.10, $q^{b}(t, x, y)$ is continuous and $q^{b}(t, x, y) \leq c_{1} e^{c_{2} t} p_{M_{b, \lambda}}(t, x, y)$ for some positive constants $c_{1}$ and $c_{2}, X^{b}$ enjoys the strong Feller property.

Proposition 5.1. Suppose that $b$ is a bounded function satisfying conditions (1.2), (1.18) and (1.19). For each $x \in \mathbb{R}^{d}$ and $f \in C_{b}^{2}\left(\mathbb{R}^{d}\right)$,

$$
M_{t}^{f}:=f\left(X_{t}^{b}\right)-f\left(X_{0}^{b}\right)-\int_{0}^{t} \mathcal{L}^{b} f\left(X_{s}^{b}\right) d s
$$

is a martingale under $\mathbb{P}_{x}$. So in particular, the Feller process $\left(X^{b}, \mathbb{P}_{x}, x \in \mathbb{R}^{d}\right)$ solves the martingale problem for $\left(\mathcal{L}^{b}, C_{\infty}^{2}\left(\mathbb{R}^{d}\right)\right)$.

Proof. This follows immediately from Theorem 3.14 and the Markov property of $X^{b}$.

We next determine the Lévy system of $X^{b}$. Recall that

$$
J^{b}(x, y)=\frac{\mathcal{A}(d,-\alpha)}{|x-y|^{d+\alpha}}+\frac{\mathcal{A}(d,-\beta) b(x, y-x)}{|x-y|^{d+\beta}} .
$$

Proposition 5.2. Suppose that $b$ is a bounded function satisfying conditions (1.2), (1.18) and (1.19). Assume that $A$ and $B$ are disjoint compact sets in $\mathbb{R}^{d}$. Then

$$
\sum_{s \leq t} \mathbb{1}_{\left\{X_{s-}^{b} \in A, X_{s}^{b} \in B\right\}}-\int_{0}^{t} \mathbb{1}_{A}\left(X_{s}^{b}\right) \int_{B} J^{b}\left(X_{s}^{b}, y\right) d y d s
$$

is a $\mathbb{P}_{x}$-martingale for each $x \in \mathbb{R}^{d}$. 
Proof. The proof is similar to that for [9, Theorem 2.6]. For reader's convenience, we give the details here. Let $f \in C^{\infty}\left(\mathbb{R}^{d}\right)$ with $f=0$ in an open neighborhood of $A$ and $f=1$ in an open neighborhood of $B$. Define

$$
M_{t}^{f}:=f\left(X_{t}^{b}\right)-f\left(X_{0}^{b}\right)-\int_{0}^{t} \mathcal{L}^{b} f\left(X_{s}^{b}\right) d s .
$$

Then $M_{t}^{f}$ is a martingale under $\mathbb{P}_{x}$ by Proposition [5.1, and so is $N_{t}^{f}:=\int_{0}^{t} \mathbb{1}_{A}\left(X_{s-}^{b}\right) d M_{s}^{f}$. Proposition 5.1 in particular implies that $X_{t}^{b}=\left(X_{t}^{b, 1}, \ldots, X_{t}^{b, d}\right)$ is a semi-martingale. So by Ito's formula, we have that,

$$
f\left(X_{t}^{b}\right)-f\left(X_{0}^{b}\right)=\sum_{i=1}^{d} \int_{0}^{t} \partial_{i} f\left(X_{s-}^{b}\right) d X_{s}^{b, i}+\sum_{s \leq t} \eta_{s}(f)+\frac{1}{2} A_{t}(f),
$$

where

$$
\eta_{s}(f)=f\left(X_{s}^{b}\right)-f\left(X_{s-}^{b}\right)-\sum_{i=1}^{d} \partial_{i} f\left(X_{s-}^{b}\right)\left(X_{s}^{b, i}-X_{s-}^{b, i}\right)
$$

and

$$
A_{t}(f)=\sum_{i, j=1}^{d} \int_{0}^{t} \partial_{i} \partial_{j} f\left(X_{s-}^{b}\right) d\left\langle\left(X^{b, i}\right)^{c},\left(X^{b, j}\right)^{c}\right\rangle_{s} .
$$

Since $f$ vanishes in an open neighborhood of $A$, we have by (5.2)-(5.4), (1.1) and (1.3) that

$$
\begin{aligned}
N_{t}^{f} & =\sum_{s \leq t} \mathbf{1}_{A}\left(X_{s-}^{b}\right) f\left(X_{s}^{b}\right)-\int_{0}^{t} \mathbf{1}_{A}\left(X_{s}^{b}\right)\left(\mathcal{L}^{b} f\left(X_{s}^{b}\right)\right) d s \\
& =\sum_{s \leq t} \mathbf{1}_{A}\left(X_{s-}^{b}\right) f\left(X_{s}^{b}\right)-\int_{0}^{t} \mathbf{1}_{A}\left(X_{s}^{b}\right) \int_{\mathbb{R}^{d}} f(y) J^{b}\left(X_{s}^{b}, y\right) d y d s .
\end{aligned}
$$

By taking a sequence of functions $f_{n} \in C_{c}^{\infty}\left(\mathbb{R}^{d}\right)$ with $f_{n}=0$ on $A, f_{n}=1$ on $B$ and $f_{n} \downarrow \mathbf{1}_{B}$, we get that, for any $x \in \mathbb{R}^{d}$,

$$
\sum_{s \leq t} \mathbf{1}_{A}\left(X_{s-}^{b}\right) \mathbf{1}_{B}\left(X_{s}^{b}\right)-\int_{0}^{t} \mathbf{1}_{A}\left(X_{s}^{b}\right) \int_{B} J^{b}\left(X_{s}^{b}, y\right) d y d s
$$

is a $\mathbb{P}_{x}$-martingale for every $x \in \mathbb{R}^{d}$.

Proposition 5.2 implies that

$$
\mathbb{E}_{x}\left[\sum_{s \leq t} \mathbf{1}_{A}\left(X_{s-}^{b}\right) \mathbf{1}_{B}\left(X_{s}^{b}\right)\right]=\mathbb{E}_{x}\left[\int_{0}^{t} \int_{\mathbb{R}^{d}} \mathbf{1}_{A}\left(X_{s}^{b}\right) \mathbf{1}_{B}(y) J^{b}\left(X_{s}^{b}, y\right) d y d s\right] .
$$

Using this and a routine measure theoretic argument, we get

$$
\mathbb{E}_{x}\left[\sum_{s \leq t} f\left(s, X_{s-}^{b}, X_{s}^{b}\right)\right]=\mathbb{E}_{x}\left[\int_{0}^{t} \int_{\mathbb{R}^{d}} f\left(s, X_{s}^{b}, y\right) J^{b}\left(X_{s}^{b}, y\right) d y d s\right]
$$

for any non-negative measurable function $f$ on $(0, \infty) \times \mathbb{R}^{d} \times \mathbb{R}^{d}$ vanishing on $\left\{(x, y) \in \mathbb{R}^{d} \times \mathbb{R}^{d}\right.$ : $x=y\}$. Finally, following the same arguments as in [11, Lemma 4.7] and [12, Appendix A], we get 
Proposition 5.3. Suppose that $b$ is a bounded function satisfying conditions (1.2), (1.18) and (1.19). Let $f$ be a nonnegative function on $\mathbb{R}_{+} \times \mathbb{R}^{d} \times \mathbb{R}^{d}$ vanishing on the diagonal. Then for stopping time $T$ with respect to the minimal admissible filtration generated by $X^{b}$,

$$
\mathbb{E}_{x}\left[\sum_{s \leq T} f\left(s, X_{s-}^{b}, X_{s}^{b}\right)\right]=\mathbb{E}_{x}\left[\int_{0}^{T} \int_{\mathbb{R}^{d}} f\left(s, X_{s}^{b}, u\right) J^{b}\left(X_{s}^{b}, u\right) d u d s\right]
$$

To remove the assumption (1.18) on $b$, we approximate a general measurable function $b(x, z)$ by continuous $k_{n}(x, z)$. To show that $q^{k_{n}}(t, x, y)$ converges to $q^{b}(t, x, y)$, we establish equicontinuity of $q^{b}(t, x, y)$ and apply the uniqueness result, Theorem 3.10 .

Proposition 5.4. For each $0<t_{0}<T<\infty$ and $A>0$, the function $q^{b}(t, x, y)$ is uniformly continuous in $(t, x) \in\left(t_{0}, T\right) \times \mathbb{R}^{d}$ for every $b$ with $\|b\|_{\infty} \leq A$ that satisfies (1.2) and for all $y \in \mathbb{R}^{d}$.

Proof. In view of Theorem [3.12, it suffices to prove the theorem for $A=A_{0}$, where $A_{0}$ is the constant in Lemma 3.4 (or in Theorem 1.1). Using the Chapman-Kolmogorov equation for $q^{b}(t, x, y)$ (see Lemma 3.13) and (3.34), it suffices to prove the Proposition for $T=1$.

Noting that by (3.8)

$$
q_{n}^{b}(t, x, y)=\int_{0}^{t} \int_{\mathbb{R}^{d}} p_{0}(t-r, x, z) \mathcal{S}_{z}^{b} q_{n-1}^{b}(r, z, y) d z d r .
$$

Hence, for $T>t>s>t_{0}, x_{1}, x_{2} \in \mathbb{R}^{d}$ and $y \in \mathbb{R}^{d}$, we have

$$
\begin{aligned}
& \left|q_{n}^{b}\left(s, x_{1}, y\right)-q_{n}^{b}\left(t, x_{2}, y\right)\right| \\
\leq & \int_{0}^{s} \int_{\mathbb{R}^{d}}\left|p_{0}\left(s-r, x_{1}, z\right)-p_{0}\left(t-r, x_{2}, z\right)\right|\left|\mathcal{S}_{z}^{b} q_{n-1}^{b}(r, z, y)\right| d z d r \\
& +\int_{s}^{t} \int_{\mathbb{R}^{d}} p_{0}\left(t-r, x_{2}, z\right)\left|\mathcal{S}_{z}^{b} q_{n-1}^{b}(r, z, y)\right| d z d r \\
= & : I+I I .
\end{aligned}
$$

It is known (see [11]) that there are positive constants $c_{1}$ and $\theta$ so that for any $t, s \in\left[t_{0}, T\right]$ and $x_{i} \in \mathbb{R}^{d}$ with $i=1,2$,

$$
\left|p_{0}\left(s, x_{1}, y\right)-p_{0}\left(t, x_{2}, y\right)\right| \leq c_{1} t_{0}^{-(d+\theta) / \alpha}\left(|t-s|^{1 / \alpha}+\left|x_{1}-x_{2}\right|\right)^{\theta}, \quad y \in \mathbb{R}^{d},
$$

we have by (2.5), (3.15) and (3.39), for $\rho \in(0, s / 2)$,

$$
\begin{aligned}
I= & \int_{0}^{s-\rho} \int_{\mathbb{R}^{d}}\left|p_{0}\left(s-r, x_{1}, z\right)-p_{0}\left(t-r, x_{2}, z\right)\right|\left|\mathcal{S}_{z}^{b} q_{n-1}^{b}(r, z, y)\right| d z d r \\
& +\int_{s-\rho}^{s} \int_{\mathbb{R}^{d}}\left|p_{0}\left(s-r, x_{1}, z\right)-p_{0}\left(t-r, x_{2}, z\right)\right|\left|\mathcal{S}_{z}^{b} q_{n-1}^{b}(r, z, y)\right| d z d r \\
\leq & c_{2} 2^{-(n-1)} \rho^{-(d+\theta) / \alpha}\left(|t-s|^{1 / \alpha}+\left|x_{1}-x_{2}\right|\right)^{\theta} \int_{0}^{s-\rho} \int_{\mathbb{R}^{d}} f_{0}(r, z, y) d z d r \\
& +c_{2} 2^{-(n-1)} \int_{s-\rho}^{s} \int_{\mathbb{R}^{d}}\left(p_{0}\left(s-r, x_{1}, z\right)+p_{0}\left(t-r, x_{2}, z\right)\right) f_{0}(r, z, y) d z d r \\
\leq & c_{3} 2^{-(n-1)} \rho^{-(d+\theta) / \alpha}\left(|t-s|^{1 / \alpha}+\left|x_{1}-x_{2}\right|\right)^{\theta} s^{1-\beta / \alpha}+c_{3} 2^{-(n-1)}(s-\rho)^{-(d+\beta) / \alpha} \rho .
\end{aligned}
$$


Moreover, by (2.5) and (3.15),

$$
I I \leq 2^{-(n-1)} \int_{s}^{t} \int_{\mathbb{R}^{d}} p_{0}\left(t-r, x_{2}, z\right) f_{0}(r, z, y) d z d r \leq 2^{-(n-1)} s^{-(d+\beta) / \alpha}|t-s| .
$$

Therefore, noting that

$$
\left|q^{b}\left(s, x_{1}, y\right)-q^{b}\left(t, x_{2}, y\right)\right| \leq\left|p_{0}\left(s, x_{1}, y\right)-p_{0}\left(t, x_{2}, y\right)\right|+\sum_{n=1}^{\infty}\left|q_{n}^{b}\left(s, x_{1}, y\right)-q_{n}^{b}\left(t, x_{2}, y\right)\right|,
$$

then first taking $|t-s|$ and $\left|x_{1}-x_{2}\right|$ small, and then making $\rho$ small in (5.5) and (5.6) yields the conclusion of this Proposition.

Proposition 5.5. For each $0<t_{0}<T<\infty$ and $A>0$, the function $q^{b}(t, x, y)$ is uniformly continuous in $y$ for every $b$ with $\|b\|_{\infty} \leq A$ that satisfies (1.2) and for all $(t, x) \in\left(t_{0}, T\right) \times \mathbb{R}^{d}$.

Proof. In view of Theorem 3.12, it suffices to prove the theorem for $A=A_{0}$, where $A_{0}$ is the constant in Lemma 3.4 (or in Theorem 1.1). Using the Chapman-Kolmogorov equation for $q^{b}(t, x, y)$ (see Lemma 3.13) and (3.34), it suffices to prove the Proposition for $T=1$.

Define $P(s, x, y)=p_{0}(s, x)-p_{0}(s, y)$. For $s>0$, we have

$$
\begin{aligned}
& \left|\mathcal{S}^{b} p_{0}\left(s, y_{1}\right)-\mathcal{S}^{b} p_{0}\left(s, y_{2}\right)\right| \\
\leq & c_{1} \int_{\mathbb{R}^{d}}\left|P\left(s, y_{1}+h, y_{2}+h\right)-P\left(s, y_{1}, y_{2}\right)-\left\langle\nabla_{\left(y_{1}, y_{2}\right)} P\left(s, y_{1}, y_{2}\right), h \mathbb{1}_{|h| \leq 1}\right\rangle\right| \frac{d h}{|h|^{d+\beta}} \\
\leq & c_{1} \int_{|h| \leq 1}|h|^{2} \sup _{\theta \in(0,1)}\left|\frac{\partial^{2}}{\partial y_{1}^{2}} p_{0}\left(s, y_{1}+\theta h\right)-\frac{\partial^{2}}{\partial y_{2}^{2}} p_{0}\left(s, y_{2}+\theta h\right)\right| \frac{d h}{|h|^{d+\beta}} \\
& +c_{1} \int_{|h|>1}\left|p_{0}\left(s, y_{1}+h\right)-p_{0}\left(s, y_{2}+h\right)-p_{0}\left(s, y_{1}\right)+p_{0}\left(s, y_{2}\right)\right| \frac{d h}{|h|^{d+\beta}} \\
\leq & c_{2} \sup _{y}\left|\frac{\partial^{3}}{\partial y^{3}} p_{0}(s, y)\right|\left|y_{1}-y_{2}\right| \int_{|h| \leq 1}|h|^{2} \frac{d h}{|h|^{d+\beta}}+c_{2} \sup _{y}\left|\frac{\partial}{\partial y} p_{0}(s, y)\right|\left|y_{1}-y_{2}\right| \int_{|h|>1} \frac{d h}{|h|^{d+\beta}} \\
\leq & c_{3}\left|y_{1}-y_{2}\right|\left[s^{-(d+3) / \alpha}+s^{-(d+1) / \alpha}\right],
\end{aligned}
$$

where in the fourth inequality, $\left|\frac{\partial^{3}}{\partial y^{3}} p_{0}(s, y)\right| \leq c_{3} s^{-(d+3) / \alpha}$ can be proved similarly by the argument in Lemma 2.1. Take $\rho \in\left(0, t_{0} / 2\right)$. Then for each $n \geq 1$, we have by (1.8), (3.39), Lemma 
2.4. Lemma 3.4 and (5.7), that for $(t, x, y) \in\left(t_{0}, 1\right) \times \mathbb{R}^{d} \times \mathbb{R}^{d}$,

$$
\begin{aligned}
& \left|q_{n}^{b}\left(t, x, y_{1}\right)-q_{n}^{b}\left(t, x, y_{2}\right)\right| \\
\leq & \int_{0}^{\rho} \int_{\mathbb{R}^{d}} q_{n-1}^{b}(t-s, x, z)\left|\mathcal{S}_{z}^{b} p_{0}\left(s, z, y_{1}\right)-\mathcal{S}_{z}^{b} p_{0}\left(s, z, y_{2}\right)\right| d z d s \\
& +\int_{\rho}^{t} \int_{\mathbb{R}^{d}} q_{n-1}^{b}(t-s, x, z)\left|\mathcal{S}_{z}^{b} p_{0}\left(s, z, y_{1}\right)-\mathcal{S}_{z}^{b} p_{0}\left(s, z, y_{2}\right)\right| d z d s \\
\leq & c_{4} 2^{-(n-1)} \int_{0}^{\rho} \int_{\mathbb{R}^{d}} p_{1}(t-s, x, z)\left|\mathcal{S}_{z}^{b} p_{0}\left(s, z, y_{1}\right)-\mathcal{S}_{z}^{b} p_{0}\left(s, z, y_{2}\right)\right| d z d s \\
& +c_{4} 2^{-(n-1)} \int_{\rho}^{t} \int_{\mathbb{R}^{d}} p_{1}(t-s, x, z)\left|\mathcal{S}_{z}^{b} p_{0}\left(s, z-y_{1}\right)-\mathcal{S}_{z}^{b} p_{0}\left(s, z-y_{2}\right)\right| d z d s \\
\leq & c_{5} 2^{-(n-1)} t_{0}^{-d / \alpha} \int_{0}^{\rho} \int_{\mathbb{R}^{d}}\left(\left|\mathcal{S}_{z}^{b} p_{0}\left(s, z, y_{1}\right)\right|+\left|\mathcal{S}_{z}^{b} p_{0}\left(s, z, y_{2}\right)\right|\right) d z d s \\
& +c_{5} 2^{-(n-1)} \rho^{-(d+3) / \alpha}\left|y_{1}-y_{2}\right| \int_{\rho}^{t} \int_{\mathbb{R}^{d}} p_{1}(t-s, x, z) d z d s \\
\leq & c_{6} 2^{-(n-1)} t_{0}^{-d / \alpha} \rho^{1-\beta / \alpha}+c_{6} 2^{-(n-1)} \rho^{-(d+3) / \alpha}\left|y_{1}-y_{2}\right| .
\end{aligned}
$$

Therefore we have

$$
\begin{aligned}
& \left|q^{b}\left(t, x, y_{1}\right)-q^{b}\left(t, x, y_{2}\right)\right| \\
\leq & \left|p_{0}\left(t, x, y_{1}\right)-p_{0}\left(t, x, y_{2}\right)\right|+\sum_{n=1}^{\infty} c_{6} 2^{-(n-1)} t_{0}^{-d / \alpha} \rho^{1-\beta / \alpha}+\sum_{n=1}^{\infty} c_{6} 2^{-(n-1)} \rho^{-(d+3) / \alpha}\left|y_{1}-y_{2}\right| .
\end{aligned}
$$

By first taking $\left|y_{1}-y_{2}\right|$ small and then making $\rho$ small yields the desired uniform continuity of $q^{b}(t, x, y)$.

Theorem 5.6. Suppose $b$ is a bounded function on $\mathbb{R}^{d} \times \mathbb{R}^{d}$ satisfying (1.2) and (1.19). The kernel $q^{b}(t, x, y)$ uniquely determines a Feller process $X^{b}=\left(X_{t}^{b}, t \geq 0, \mathbb{P}_{x}, x \in \mathbb{R}^{d}\right)$ on the canonical Skorokhod space $\mathbb{D}\left([0, \infty), \mathbb{R}^{d}\right)$ such that

$$
\mathbb{E}_{x}\left[f\left(X_{t}^{b}\right)\right]=\int_{\mathbb{R}^{d}} q^{b}(t, x, y) f(y) d y
$$

for every bounded continuous function $f$ on $\mathbb{R}^{d}$. The Feller process $X^{b}$ is conservative and has a Lévy system $\left(J^{b}(x, y) d y, t\right)$, where

$$
J^{b}(x, y)=\frac{\mathcal{A}(d,-\alpha)}{|x-y|^{d+\alpha}}+\frac{\mathcal{A}(d,-\beta) b(x, y-x)}{|x-y|^{d+\beta}} .
$$

Moreover, for each $x \in \mathbb{R}^{d},\left(X^{b}, \mathbb{P}_{x}\right)$ is the unique solution to the martingale problem $\left(\mathcal{L}^{b}, \mathcal{S}\left(\mathbb{R}^{d}\right)\right)$ with initial value $x$. Here $\mathcal{S}\left(\mathbb{R}^{d}\right)$ denotes the space of tempered functions on $\mathbb{R}^{d}$.

Proof. When $b$ is a bounded function satisfying (1.2), (1.18) and (1.19), the theorem has already been established via Propositions 5.1 5.3. We now remove the assumption (1.18). Suppose that $b(x, z)$ is a bounded function that satisfies (1.2) and (1.19). Let $\varphi$ be a non-negative smooth function with compact support in $\mathbb{R}^{d}$ so that $\int_{\mathbb{R}^{d}} \varphi(x) d x=1$. For each $n \geq 1$, define $\varphi_{n}(x)=n^{d} \varphi(n x)$ and

$$
k_{n}(x, z):=\int_{\mathbb{R}^{d}} \varphi_{n}(x-y) b(y, z) d y .
$$


Then $k_{n}$ is a function that satisfies (1.2), (1.18) and (1.19) with $\left\|k_{n}\right\|_{\infty} \leq\|b\|_{\infty}$. By Theorem 1.1. Proposition 5.4 and Proposition [5.5, $q^{k_{n}}(t, x, y)$ is uniformly bounded and equi-continuous on $[1 / M, M] \times \mathbb{R}^{d} \times \mathbb{R}^{d}$ for each $M \geq 1$, then there is a subsequence $\left\{n_{j}\right\}$ of $\{n\}$ so that $q^{k_{n_{j}}}(t, x, y)$ converges boundedly and uniformly on compacts of $(0, \infty) \times \mathbb{R}^{d} \times \mathbb{R}^{d}$, to some continuous function $\bar{q}(t, x, y)$, which again satisfies (1.16). Obviously, $\bar{q}(t, x, y)$ also satisfies the Chapman-Kolmogorov equation and $\int_{\mathbb{R}^{d}} \bar{q}(t, x, y) d y=1$. By (3.29) and Theorem 3.7,

$$
q^{k_{n_{j}}}(t, x, y)=p_{0}(t, x, y)+\int_{0}^{t} \int_{\mathbb{R}^{d}} q^{k_{n_{j}}}(t-s, x, z) \mathcal{S}_{z}^{k_{n_{j}}} p_{0}(s, z, y) d z d s
$$

and

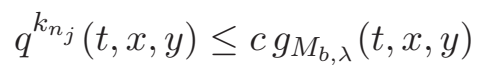

for every $0<t \leq 1 \wedge\left(A_{0} /\|b\|_{\infty}\right)^{\alpha /(\alpha-\beta)}$ and $x, y \in \mathbb{R}^{d}$, where $c$ is a positive constant that depends only on $d, \alpha, \beta$ and $\|b\|_{\infty}$. Letting $j \rightarrow \infty$, we have by (3.1), Lemma 2.5 and the dominated convergence theorem that

$$
\bar{q}(t, x, y)=p_{0}(t, x, y)+\int_{0}^{t} \int_{\mathbb{R}^{d}} \bar{q}(t-s, x, z) \mathcal{S}_{z}^{b} p_{0}(s, z, y) d y d s
$$

and $\bar{q}(t, x, y) \leq c g_{M_{b, \lambda}}(t, x, y)$ for every $0<t \leq 1 \wedge\left(A_{0} /\|b\|_{\infty}\right)^{\alpha /(\alpha-\beta)}$ and $x, y \in \mathbb{R}^{d}$. Hence we conclude from Theorem 3.10 that $\bar{q}(t, x, y)=q^{b}(t, x, y)$. This in particular implies that $q^{b}(t, x, y) \geq 0$. So there is a Feller process $X^{b}$ having $q^{b}(t, x, y)$ as its transition density function. The proof of Propositions 5.1 5.3 only uses the condition (1.18) through its implication that $q^{b}(t, x, y) \geq 0$. So in view of what we just established, Propositions 5.155.3 continue to hold for $X^{b}$ under the current setting without the additional assumption (1.18). The non-local operator $\mathcal{L}^{b}$ satisfies the assumptions $\left[A_{1}\right]$ and $\left[A_{2}\right]$ of [20]. So by [20, Theorem 3], solution to the martingale problem $\left(\mathcal{L}^{b}, \mathcal{S}\left(\mathbb{R}^{d}\right)\right)$ is unique. Since $\mathcal{S}\left(\mathbb{R}^{d}\right) \subset C_{\infty}^{2}\left(\mathbb{R}^{d}\right)$, the proof of the theorem is now complete.

For each $\lambda>0$, define

$$
\widehat{b}_{\lambda}(x, z)=b(x, z) 1_{\{|z| \leq \lambda\}}(z)+b^{+}(x, z) 1_{\{|z|>\lambda\}}(z) .
$$

In the following, we use a method of Meyer [21] to construct from $X^{b}$, by adding suitable jumps, a strong Markov process $Y$ corresponding to the jumping kernel $J^{\widehat{b}_{\lambda}}$ defined by (1.25) but with $\widehat{b}_{\lambda}$ in place of $b$. Define

$$
\mathcal{J}(x)=\int_{\mathbb{R}^{d}}\left(\widehat{J}^{\widehat{b}_{\lambda}}(x, y)-J^{b}(x, y)\right) d y
$$

Then there exists a positive constant $c_{1}$ so that $0 \leq \mathcal{J}(x) \leq c_{1}$ for all $x \in \mathbb{R}^{d}$. Let

$$
q(x, y)=\frac{J^{\widehat{b}_{\lambda}}(x, y)-J^{b}(x, y)}{\mathcal{J}(x)} .
$$

Let $S_{1}$ be an exponential random variable of parameter 1 independent of $X^{b}$. Set

$$
C_{t}=\int_{0}^{t} \mathcal{J}\left(X_{s}^{b}\right) d s, \quad U_{1}=\inf \left\{t \geq 0: C_{t} \geq S_{1}\right\}
$$

We let $Y_{t}=X_{t}^{b}$ for $0 \leq t<U_{1}$ and define $Y_{U_{1}}$ with law $q\left(Y_{U_{1-}}, \cdot\right)=q\left(X_{U_{1-}}^{b}, \cdot\right)$, and then repeat using an independent exponential random variable $S_{2}$ to define $U_{2}$, etc. So the construction 
proceeds now in the same way from the new starting point $\left(U_{1}, Y_{U_{1}}\right)$. Since $\mathcal{J}(x)$ is bounded, only finitely many new jumps are introduced in any bounded time interval. In [21], it is proved that the resulting process $Y$ is a strong Markov process. By slightly abusing the notation, we still use $\mathbb{P}_{x}$ and $\mathbb{E}_{x}$ to denote the above constructed probability law and expectation induced on such enlarged probability space under which $Y_{0}=x$.

Lemma 5.7. For each $x \in \mathbb{R}^{d}$ and $f \in C_{b}^{2}\left(\mathbb{R}^{d}\right)$,

$$
\mathbb{E}_{x}\left[f\left(Y_{t}\right) ; t<U_{1}\right]=f(x)+\mathbb{E}_{x}\left[\int_{0}^{t}\left(\mathcal{L}^{b}-\mathcal{J}\left(Y_{s}\right)\right) f\left(Y_{s}\right) \mathbb{1}_{\left\{s<U_{1}\right\}} d s\right]
$$

Proof. By the definition of $U_{1}$ and Ito's formula, for each function $f \in C_{b}^{2}\left(\mathbb{R}^{d}\right)$,

$$
\begin{aligned}
\mathbb{E}_{x}\left[f\left(Y_{t}\right) ; t<U_{1}\right] & =\mathbb{E}_{x}\left[f\left(X_{t}^{b}\right) \mathbb{1}_{\left\{U_{1}>t\right\}}\right]=\mathbb{E}_{x}\left[f\left(X_{t}^{b}\right) e^{-C_{t}}\right] \\
& =f(x)+\mathbb{E}_{x}\left[\int_{0}^{t}\left(\mathcal{L}^{b}-\mathcal{J}\left(X_{s}^{b}\right)\right) f\left(X_{s}^{b}\right) e^{-C_{s}} d s\right] \\
& =f(x)+\mathbb{E}_{x}\left[\int_{0}^{t}\left(\mathcal{L}^{b}-\mathcal{J}\left(Y_{s}\right)\right) f\left(Y_{s}\right) \mathbb{1}_{\left\{s<U_{1}\right\}} d s\right]
\end{aligned}
$$

Proposition 5.8. For each $x \in \mathbb{R}^{d}$ and $f \in C_{b}^{2}\left(\mathbb{R}^{d}\right)$,

$$
M_{t}^{f}:=f\left(Y_{t}\right)-f\left(Y_{0}\right)-\int_{0}^{t} \mathcal{L}^{\widehat{b}_{\lambda}} f\left(Y_{s}\right) d s
$$

is a martingale under $\mathbb{P}_{x}$. So in particular, the strongly Markov process $\left(Y, \mathbb{P}_{x}, x \in \mathbb{R}^{d}\right)$ solves the martingale problem for $\left(\mathcal{L}^{\widehat{b}_{\lambda}}, C_{\infty}^{2}\left(\mathbb{R}^{d}\right)\right)$.

Proof. Note that $M_{t}^{f}$ is an additive function of $Y$. So by the Markov property of $Y$, it suffices to show that $\mathbb{E}_{x}\left[M_{t}^{f}\right]=0$ for every $x \in \mathbb{R}^{d}$ and $t>0$.

Recall that $U_{1}$ is defined in (5.9), and denote by $\left\{U_{n}, n \geq 2\right\}$ the subsequent jump adding times inductively defined according to the construction of Meyer [21]. For every $\alpha>0$, set $u_{\alpha}(x)=\mathbb{E}_{x}\left[\int_{0}^{U_{1}} e^{-\alpha t} f\left(Y_{t}\right) d t\right]$. Since by Lemma [5.7.

$$
\mathbb{E}_{x}\left[f\left(Y_{t}\right) ; t<U_{1}\right]=f(x)+\mathbb{E}_{x}\left[\int_{0}^{t}\left(\mathcal{L}^{b}-\mathcal{J}\left(Y_{s}\right)\right) f\left(Y_{s}\right) \mathbb{1}_{\left\{s<U_{1}\right\}} d s\right]
$$

we have by Fubini theorem that

$$
u_{\alpha}(x)=\frac{f(x)}{\alpha}+\frac{1}{\alpha} \mathbb{E}_{x}\left[\int_{0}^{U_{1}} e^{-\alpha s}\left(\mathcal{L}^{b}-\mathcal{J}\left(Y_{s}\right)\right) f\left(Y_{s}\right) d s\right] .
$$

Observe that in view of [25, p.286] (see, for example, the proof of [14, Proposition 2.2]), for any non-negative function $\varphi$ on $\mathbb{R}^{d}$ and $x \in \mathbb{R}^{d}$,

$$
\mathbb{E}_{x}\left[e^{-\alpha U_{1}} \varphi\left(Y_{U_{1}-}\right)\right]=\mathbb{E}_{x}\left[\int_{0}^{U_{1}} e^{-\alpha s} \mathcal{J}\left(Y_{s}\right) \varphi\left(Y_{s}\right) d s\right] .
$$


Set $U_{0}=0$ and let $\theta_{t}$ to denote the time shift operator for the Markov process $Y$. Then we have from above and the strong Markov property of $Y$ that

$$
\begin{aligned}
& \mathbb{E}_{x}\left[\int_{0}^{\infty} e^{-\alpha t} f\left(Y_{t}\right) d t\right]=\sum_{j=0}^{\infty} \mathbb{E}_{x}\left[\int_{U_{j}}^{U_{j+1}} e^{-\alpha t} f\left(Y_{t}\right) d t\right]=\sum_{j=0}^{\infty} \mathbb{E}_{x}\left[e^{-\alpha U_{j}} u_{\alpha}\left(Y_{U_{j}}\right)\right] \\
= & \frac{f(x)}{\alpha}+\frac{1}{\alpha} \sum_{j=1}^{\infty} \mathbb{E}_{x}\left[e^{-\alpha U_{j}} f\left(Y_{U_{j}}\right)\right]+\frac{1}{\alpha} \sum_{j=0}^{\infty} \mathbb{E}_{x}\left[\int_{U_{j}}^{U_{j+1}} e^{-\alpha s}\left(\mathcal{L}^{b}-\mathcal{J}\left(Y_{s}\right)\right) f\left(Y_{s}\right) d s\right] \\
= & \frac{f(x)}{\alpha}+\frac{1}{\alpha} \sum_{j=1}^{\infty} \mathbb{E}_{x}\left[e^{-\alpha U_{j}} \int_{\mathbb{R}^{d}} f(y) q\left(Y_{U_{j}-}, y\right) d y\right]+\frac{1}{\alpha} \mathbb{E}_{x}\left[\int_{0}^{\infty} e^{-\alpha s}\left(\mathcal{L}^{b}-\mathcal{J}\left(Y_{s}\right)\right) f\left(Y_{s}\right) d s\right] \\
= & \frac{f(x)}{\alpha}+\frac{1}{\alpha} \sum_{j=1}^{\infty} \mathbb{E}_{x}\left[e^{-\alpha U_{j-1}} \int_{\mathbb{R}^{d}} f(y)\left(e^{-\alpha U_{1}} q\left(Y_{U_{1}-}, y\right)\right) \circ \theta_{U_{j-1}} d y\right] \\
& +\frac{1}{\alpha} \mathbb{E}_{x}\left[\int_{0}^{\infty} e^{-\alpha s}\left(\mathcal{L}^{b}-\mathcal{J}\left(Y_{s}\right)\right) f\left(Y_{s}\right) d s\right] \\
= & \frac{f(x)}{\alpha}+\frac{1}{\alpha} \sum_{j=1}^{\infty} \mathbb{E}_{x}\left[e^{-\alpha U_{j-1}} \int_{\mathbb{R}^{d}} f(y)\left(\int_{0}^{U_{1}} e^{-\alpha s} \mathcal{J}\left(Y_{s}\right) q\left(Y_{s}, y\right) d s\right) \circ \theta_{U_{j-1}} d y\right] \\
& +\frac{1}{\alpha} \mathbb{E}_{x}\left[\int_{0}^{\infty} e^{-\alpha s}\left(\mathcal{L}^{b}-\mathcal{J}\left(Y_{s}\right)\right) f\left(Y_{s}\right) d s\right] \\
= & \frac{f(x)}{\alpha}+\frac{1}{\alpha} \mathbb{E}_{x}\left[\int_{\mathbb{R}^{d}} f(y)\left(\int_{0}^{\infty} e^{-\alpha s} \mathcal{J}\left(Y_{s}\right) q\left(Y_{s}, y\right) d s\right) d y\right] \\
& +\frac{1}{\alpha} \mathbb{E}_{x}\left[\int_{0}^{\infty} e^{-\alpha s}\left(\mathcal{L}^{b}-\mathcal{J}\left(Y_{s}\right)\right) f\left(Y_{s}\right) d s\right] \\
= & \frac{f(x)}{\alpha}+\frac{1}{\alpha} \mathbb{E}_{x}\left[\int_{0}^{\infty} e^{-\alpha s}\left(\mathcal{L}^{b} f\left(Y_{s}\right)+\int_{\mathbb{R}^{d}} \mathcal{J}\left(Y_{s}\right) q\left(Y_{s}, y\right)\left(f(y)-f\left(Y_{s}\right)\right) d y\right) d s\right] \\
= & \frac{f(x)}{\alpha}+\frac{1}{\alpha} \mathbb{E}_{x}\left[\int_{0}^{\infty} e^{-\alpha s} \widehat{\mathcal{L}}^{b_{\lambda}} f\left(Y_{s}\right) d s\right] . \\
&
\end{aligned}
$$

By the uniqueness of the Laplace transform, we conclude from above that $\mathbb{E}_{x}\left[M_{t}^{f}\right]=0$ for all $t \geq 0$ and $x \in \mathbb{R}^{d}$.

Note that $\widehat{b}_{\lambda}$ defined by (5.8) is a bounded function on $\mathbb{R}^{d} \times \mathbb{R}^{d}$ satisfying (1.2) and (1.19). By Theorem 5.6, the kernel $q^{\widehat{b}_{\lambda}}(t, x, y)$ uniquely determines a Feller process $X^{\widehat{b}_{\lambda}}=\left(X_{t}^{\widehat{b}_{\lambda}}, t \geq\right.$ $\left.0, \mathbb{P}_{x}, x \in \mathbb{R}^{d}\right)$ on the canonical Skorokhod space $\mathbb{D}\left([0, \infty), \mathbb{R}^{d}\right)$, and $\left(X^{\widehat{b}_{\lambda}}, \mathbb{P}_{x}\right)$ is the unique solution to the martingale problem for $\left(\mathcal{L}^{b_{\lambda}}, \mathcal{S}\left(\mathbb{R}^{d}\right)\right)$ with initial value $x$. This, together with Proposition 5.8 implies that the process $Y$ coincides with $X^{\widehat{b}_{\lambda}}$ in the sense of distribution.

Theorem 5.9. For every $\lambda>0$ and $A>0$, there is a positive constant $C_{15}=C_{15}(d, \alpha, \beta, A, \lambda)$ such that for any bounded $b$ satisfying (1.2) and (1.19) with $\|b\|_{\infty} \leq A$,

$$
q^{b}(t, x, y) \leq C_{15} p_{M_{b^{+}, \lambda}}(t, x, y) \quad \text { for } t \in(0,1] \text { and } x, y \in \mathbb{R}^{d} .
$$

Proof. Noting that $\widehat{b}_{\lambda}$ is a bounded function on $\mathbb{R}^{d} \times \mathbb{R}^{d}$ with $\left\|\widehat{b}_{\lambda}\right\|_{\infty} \leq\|b\|_{\infty}$ satisfying (1.2) and (1.19), then by Theorem 1.1, there is a positive constant $C=C(d, \alpha, \beta, A, \lambda)$ so that

$$
q^{\widehat{b}_{\lambda}}(t, x, y) \leq C p_{M_{b^{+}, \lambda}}(t, x, y) \quad \text { for } t \in(0,1] \text { and } x, y \in \mathbb{R}^{d} .
$$


Let $\left\{\mathcal{M}_{t}\right\}_{t \geq 0}$ be the filtration generated by $X^{b}$. Note that $X^{\widehat{b}_{\lambda}}$ has the same distribution as $Y$. Then by Lemma 3.6 in [2], for any $A \in \mathcal{M}_{t}$,

$$
\mathbb{P}^{x}\left(X_{t}^{\widehat{b}_{\lambda}} \in A\right)=\mathbb{P}^{x}\left(Y_{t} \in A\right) \geq \mathbb{P}^{x}\left(\left\{Y_{s}=X_{s}^{b} \text { for all } 0 \leq s \leq t\right\} \cap A\right) \geq e^{-t\|\mathcal{J}\|_{\infty} \mathbb{P}^{x}}\left(X_{t}^{b} \in A\right) .
$$

Hence, by (5.10)

$$
q^{b}(t, x, y) \leq e^{\|\mathcal{J}\|_{\infty}} q^{\widehat{b}_{\lambda}}(t, x, y) \leq C e^{\|\mathcal{J}\|_{\infty}} p_{M_{b^{+}, \lambda}}(t, x, y) \quad \text { for } t \in(0,1] \text { and } x, y \in \mathbb{R}^{d} .
$$

For a Borel set $B \subset \mathbb{R}^{d}$, we define $\tau_{B}^{b}=\inf \left\{t>0: X_{t}^{b} \notin B\right\}$ and $\sigma_{B}^{b}:=\inf \left\{t \geq 0: X_{t}^{b} \in B\right\}$.

Proposition 5.10. For each $A>0$ and $R_{0}>0$, there exists a positive constant

$$
\kappa=\kappa\left(d, \alpha, \beta, A, R_{0}\right)<2^{\alpha}\left(1-(1 / 3)^{\alpha}\right)
$$

so that for every $b$ satisfying (1.2) and (1.19) with $\|b\|_{\infty} \leq A, r \in\left(0, R_{0}\right]$ and $x \in \mathbb{R}^{d}$,

$$
\mathbb{P}_{x}\left(\tau_{B(x, r)}^{b} \leq \kappa r^{\alpha}\right) \leq \frac{1}{2}
$$

Proof. Let $f$ be a $C^{2}$ function taking values in $[0,1]$ such that $f(0)=0$ and $f(u)=1$ if $|u| \geq 1$. Set $f_{x, r}(y)=f\left(\frac{y-x}{r}\right)$. Note that $f_{x, r}$ is a $C^{2}$ function taking values in $[0,1]$ such that $f_{x, r}(x)=0$ and $f_{x, r}(y)=1$ if $y \notin B(x, r)$. Moreover,

$$
\sup _{y \in \mathbb{R}^{d}}\left|\frac{\partial^{2} f_{x, r}(y)}{\partial y_{i} \partial y_{j}}\right| \leq r^{-2} \sup _{y \in \mathbb{R}^{d}}\left|\frac{\partial^{2} f(y)}{\partial y_{i} \partial y_{j}}\right| .
$$

Denote $\sum_{i, j=1}^{d}\left|\partial_{i j}^{2} f(x)\right|$ by $\left|D^{2} f(x)\right|$. By Taylor's formula, it follows that

$$
\begin{aligned}
\left|\mathcal{L}^{b} f_{x, r}(u)\right| \leq & c_{1} \int\left|f_{x, r}(u+h)-f_{x, r}(u)-\left\langle\nabla f_{x, r}(u), h\right\rangle \mathbb{1}_{\{|h| \leq r\}}\right|\left(\frac{1}{|h|^{d+\alpha}}+\frac{1}{|h|^{d+\beta}}\right) d h \\
= & c_{1} \int_{\{|h| \leq r\}}\left|f_{x, r}(u+h)-f_{x, r}(u)-\left\langle\nabla f_{x, r}(u), h\right\rangle\right|\left(\frac{1}{|h|^{d+\alpha}}+\frac{1}{|h|^{d+\beta}}\right) d h \\
& +c_{1} \int_{\{|h|>r\}}\left|f_{x, r}(u+h)-f_{x, r}(u)\right|\left(\frac{1}{|h|^{d+\alpha}}+\frac{1}{|h|^{d+\beta}}\right) d h \\
\leq & c_{2}\left\|D^{2} f\right\|_{\infty} r^{-2} \int_{|h| \leq r}|h|^{2}\left(\frac{1}{|h|^{d+\alpha}}+\frac{1}{|h|^{d+\beta}}\right) d h \\
& +c_{2}\|f\|_{\infty} \int_{\{|h|>r\}}\left(\frac{1}{|h|^{d+\alpha}}+\frac{1}{|h|^{d+\beta}}\right) d h \\
\leq & c_{3}\left(r^{-\alpha}+r^{-\beta}\right) \leq c_{3}\left(1+R_{0}^{\alpha-\beta}\right) r^{-\alpha},
\end{aligned}
$$

where $c_{i}=c_{i}(d, \alpha, \beta, A), i=1,2,3$ are positive constants. Therefore, for each $t>0$,

$$
\begin{aligned}
\mathbb{P}_{x}\left(\tau_{B(x, r)}^{b} \leq t\right) & \leq \mathbb{E}_{x}\left[f_{x, r}\left(X_{\tau_{B(x, r)}^{b} \wedge t}^{b}\right)\right]-f_{x, r}(x) \\
& =\mathbb{E}_{x}\left[\int_{0}^{\tau_{B(x, r)}^{b} \wedge t} \mathcal{L}^{b} f_{x, r}\left(X_{s}^{b}\right) d s\right] \leq c_{3}\left(1+R_{0}^{\alpha-\beta}\right) \frac{t}{r^{\alpha}}
\end{aligned}
$$


Set $\kappa=\left(2^{\alpha}\left[1-(1 / 3)^{\alpha}\right]\right) \wedge\left(2 c_{3}\left(1+R_{0}^{\alpha-\beta}\right)\right)^{-1}$, then

$$
\mathbb{P}_{x}\left(\tau_{B(x, r)}^{b} \leq \kappa r^{\alpha}\right) \leq \frac{1}{2}
$$

Recall that $m_{b, \lambda}=\operatorname{essinf}_{x, z \in \mathbb{R}^{d},|z|>\lambda} b(x, z)$.

Proposition 5.11. For every $A>0, \lambda>0,0<\varepsilon<1$ and $R_{0}>0$, there exists a constant $C_{16}=C_{16}\left(d, \alpha, \beta, A, \lambda, \varepsilon, R_{0}\right)>0$ so that for every $b$ satisfying (1.2) and (1.23) with $\|b\|_{\infty} \leq A$, $r \in\left(0, R_{0}\right]$ and $x, y \in \mathbb{R}^{d}$ with $|x-y| \geq 3 r$,

$$
\mathbb{P}_{x}\left(\sigma_{B(y, r)}^{b}<\kappa r^{\alpha}\right) \geq C_{16} r^{d+\alpha}\left(\frac{1}{|x-y|^{d+\alpha}}+\frac{m_{b^{+}, \lambda}}{|x-y|^{d+\beta}}\right) .
$$

Proof. By Proposition 5.10,

$$
\mathbb{E}_{x}\left[\kappa r^{\alpha} \wedge \tau_{B(x, r)}^{b}\right] \geq \kappa r^{\alpha} \mathbb{P}_{x}\left(\tau_{B(x, r)}^{b} \geq \kappa r^{\alpha}\right) \geq \frac{1}{2} \kappa r^{\alpha} .
$$

Since $J^{b}(x, y) \geq m_{b^{+}, \lambda} \mathcal{A}(d,-\beta)|x-y|^{-d-\beta_{1}} \mathbb{1}_{\{|x-y|>\lambda\}}$, (1.23) implies that

$$
J^{b}(x, y) \geq \frac{1}{2}\left(\varepsilon|x-y|^{-(d+\alpha)}+m_{b^{+}, \lambda} \mathcal{A}(d,-\beta)|x-y|^{-(d+\beta)} \mathbb{1}_{\{|x-y|>\lambda\}}\right)
$$

Thus by Proposition 5.3, there are positive constants $c_{1}=c_{1}(d, \alpha, \beta)$ and $c_{2}=c_{2}\left(d, \alpha, \beta, A, \lambda, \varepsilon, R_{0}\right)$ so that

$$
\begin{aligned}
& \mathbb{P}_{x}\left(\sigma_{B(y, r)}^{b}<\kappa r^{\alpha}\right) \geq \mathbb{P}_{x}\left(X_{\kappa r^{\alpha} \wedge \tau_{B(x, r)}^{b}}^{b} \in B(y, r)\right) \\
& =\mathbb{E}_{x} \int_{0}^{\kappa r^{\alpha} \wedge \tau_{B(x, r)}^{b}} \int_{B(y, r)} J^{b}\left(X_{s}^{b}, u\right) d u d s \\
& \geq c_{1} \mathbb{E}_{x}\left[\kappa r^{\alpha} \wedge \tau_{B(x, r)}^{b}\right] \int_{B(y, r)}\left(\frac{\varepsilon}{|x-y|^{d+\alpha}}+\frac{m_{b^{+}, \lambda}}{|x-y|^{d+\beta}} \mathbb{1}_{\{|x-y|>\lambda\}}\right) d u \\
& \geq c_{2} \varepsilon \kappa r^{d+\alpha}\left(\frac{1}{|x-y|^{d+\alpha}}+\frac{m_{b^{+}, \lambda}}{|x-y|^{d+\beta}}\right) \text {. }
\end{aligned}
$$

Here in the last inequality, we used the fact that $|x-y|^{-(d+\alpha)} \geq\left(1+\lambda^{\alpha-\beta} A\right)^{-1}\left[|x-y|^{-(d+\alpha)}+\right.$ $\left.m_{b, \lambda} \cdot|x-y|^{-(d+\beta)}\right]$ for $|x-y| \leq \lambda$.

Proposition 5.12. For every $A>0$, there exists a constant $C_{17}=C_{17}(d, \alpha, \beta, A)>0$ so that for every bounded $b$ that satisfies (1.2) and (1.19) with $\|b\|_{\infty} \leq A$, and $3 r \leq|x-y| \leq R_{*}:=$ $\frac{1}{3}\left(2 A \frac{\mathcal{A}(d,-\beta)}{\mathcal{A}(d,-\alpha)}\right)^{1 /(\beta-\alpha)}$,

$$
\mathbb{P}_{x}\left(\sigma_{B(y, r)}^{b}<\kappa r^{\alpha}\right) \geq C_{17} \frac{r^{d+\alpha}}{|x-y|^{d+\alpha}} .
$$

Proof. Note that when $|x-u| \leq 3 R_{*}, \frac{1}{2} \frac{\mathcal{A}(d,-\alpha)}{\mathcal{A}(d,-\beta)}|x-u|^{\beta-\alpha} \geq A$ and so

$$
\begin{aligned}
J^{b}(x, u) & =\frac{\mathcal{A}(d,-\alpha)}{|x-u|^{d+\alpha}}+\frac{\mathcal{A}(d,-\beta) b(x, u-x)}{|x-u|^{d+\beta}} \\
& \geq \frac{\mathcal{A}(d,-\alpha)}{|x-u|^{d+\alpha}}-A \frac{\mathcal{A}(d,-\beta)}{|x-u|^{d+\beta}} \geq \frac{1}{2} \frac{\mathcal{A}(d,-\alpha)}{|x-u|^{d+\alpha}} .
\end{aligned}
$$


By Propositions 5.3 and 5.10, we have

$$
\begin{aligned}
& \mathbb{P}_{x}\left(\sigma_{B(y, r)}^{b}<\kappa r^{\alpha}\right) \geq \mathbb{P}_{x}\left(X_{\kappa r^{\alpha} \wedge \tau_{B(x, r)}^{b}}^{b} \in B(y, r)\right) \\
& =\mathbb{E}_{x} \int_{0}^{\kappa r^{\alpha} \wedge \tau_{B(x, r)}^{b}} \int_{B(y, r)} J^{b}\left(X_{s}^{b}, u\right) d u d s \\
& \geq c_{1} \mathbb{E}_{x}\left[\kappa r^{\alpha} \wedge \tau_{B(x, r)}^{b}\right] \int_{B(y, r)} \frac{1}{|x-y|^{d+\alpha}} d u \\
& \geq c_{2} \kappa r^{d+\alpha} \frac{1}{|x-y|^{d+\alpha}},
\end{aligned}
$$

where the second inequality holds due to $\left|X_{s}^{b}-u\right| \leq 3|x-y| \leq 3 R_{*}$ and (15.12).

Theorem 5.13. For every $\lambda>0, \varepsilon \in(0,1)$ and $A>0$, there are positive constants $C_{18}=$ $C_{18}(d, \alpha, \beta, A, \lambda, \varepsilon)$ and $C_{19}=C_{19}(d, \alpha, \beta, A, \lambda)$ such that for any $b$ with $\|b\|_{\infty} \leq A$ that satisfies (1.2) and (1.23),

$$
C_{18} p_{m_{b^{+}, \lambda}}(t, x, y) \leq q^{b}(t, x, y) \leq C_{19} p_{M_{b^{+}, \lambda}}(t, x, y), \quad t \in(0,1], x, y \in \mathbb{R}^{d} .
$$

Proof. Noting that the condition (1.23) in particular implies (1.19), so the upper bound estimate follows immediately from Theorem [5.9. We only need to prove the lower bound. Let $\delta_{0}:=$ $1 \wedge\left(A_{0} / A\right)^{\alpha /(\alpha-\beta)}$. (3.28) together with (1.7) also yields that for any $\|b\|_{\infty} \leq A$,

$$
q^{b}(t, x, y) \geq c_{0} t^{-d / \alpha} \quad \text { for } t \in\left(0, \delta_{0}\right] \text { and }|x-y| \leq 3 t^{1 / \alpha} \text {. }
$$

Here $c_{0}=c_{0}(d, \alpha, \beta)$ is a positive constant. For every $t \in\left(0, \delta_{0}\right]$, by Proposition 5.10 and Proposition 5.11 with $R_{0}=1, r=t^{1 / \alpha} / 2$ and the strong Markov property of the process $X^{b}$, we get for $|x-y|>3 t^{1 / \alpha}$,

$$
\begin{aligned}
& \mathbb{P}_{x}\left(X_{2^{-\alpha} \kappa t}^{b} \in B\left(y, t^{1 / \alpha}\right)\right) \\
\geq & \mathbb{P}_{x}\left(X^{b} \operatorname{hits} B\left(y, t^{1 / \alpha} / 2\right) \text { before } 2^{-\alpha} \kappa t \text { and stays there for at least } 2^{-\alpha} \kappa t \text { units of time }\right) \\
\geq & \mathbb{P}_{x}\left(\sigma_{B\left(y, t^{1 / \alpha} / 2\right)}^{b}<2^{-\alpha} \kappa t\right) \inf _{z \in B\left(y, t^{1 / \alpha} / 2\right)} \mathbb{P}_{z}\left(\tau_{B\left(y, t^{1 / \alpha}\right)}^{b} \geq 2^{-\alpha} \kappa t\right) \\
\geq & \mathbb{P}_{x}\left(\sigma_{B\left(y, t^{1 / \alpha} / 2\right)}^{b}<2^{-\alpha} \kappa t\right) \inf _{z \in B\left(y, t^{1 / \alpha} / 2\right)} \mathbb{P}_{z}\left(\tau_{B\left(z, t^{1 / \alpha} / 2\right)}^{b} \geq 2^{-\alpha} \kappa t\right) \\
\geq & c_{1} t^{(d+\alpha) / \alpha}\left(\frac{1}{|x-y|^{d+\alpha}}+\frac{m_{b^{+}, \lambda}}{|x-y|^{d+\beta}}\right) .
\end{aligned}
$$

Here $c_{1}=c_{1}(d, \alpha, \beta, A, \lambda, \varepsilon)$ is a positive constant. Hence, by (5.14) and (5.15), for $|x-y|>3 t^{1 / \alpha}$ and $t \in\left(0, \delta_{0}\right]$,

$$
\begin{aligned}
q^{b}(t, x, y) & \geq \int_{B\left(y, t^{1 / \alpha}\right)} q^{b}\left(2^{-\alpha} \kappa t, x, z\right) q^{b}\left(\left(1-2^{-\alpha} \kappa\right) t, z, y\right) d z \\
& \geq \inf _{z \in B\left(y, t^{1 / \alpha}\right)} q^{b}\left(\left(1-2^{-\alpha} \kappa\right) t, z, y\right) \mathbb{P}_{x}\left(X_{2^{-\alpha} \kappa t}^{b} \in B\left(y, t^{1 / \alpha}\right)\right) \\
& \geq c_{2} t^{-d / \alpha} t^{(d+\alpha) / \alpha}\left(\frac{1}{|x-y|^{d+\alpha}}+\frac{m_{b^{+}, \lambda}}{|x-y|^{d+\beta}}\right) \\
& \geq c_{2}\left(\frac{t}{|x-y|^{d+\alpha}}+\frac{t m_{b^{+}, \lambda}}{|x-y|^{d+\beta}}\right)
\end{aligned}
$$


where $c_{2}=c_{2}(d, \alpha, \beta, A, \lambda, \varepsilon)>0$, the third inequality holds due to $|z-y| \leq t^{1 / \alpha} \leq 3((1-$ $\left.\left.2^{-\alpha} \kappa\right) t\right)^{1 / \alpha}$ when $\kappa \leq 2^{\alpha}\left(1-3^{-\alpha}\right)$ and (5.14)-(5.15). Finally, (5.14), (5.16) together with (1.10) and the Chapman-Kolmogorov equation yields the desired lower bound estimate.

Theorem 5.14. For every $\lambda>0$ and $A>0$, there are positive constants $C_{k}=C_{k}(d, \alpha, \beta, A), k=$ 20,21 and $C_{22}=C_{22}(d, \alpha, \beta, A, \lambda)$ such that for any bounded $b$ satisfying (1.2) and (1.19) with $\|b\|_{\infty} \leq A$,

$$
C_{20} \bar{p}_{0}\left(t, C_{21} x, C_{21} y\right) \leq q^{b}(t, x, y) \leq C_{22} p_{M_{b^{+}, \lambda}}(t, x, y) \quad \text { for } t \in(0,1] \text { and } x, y \in \mathbb{R}^{d} .
$$

Proof. By Theorem [5.9, it suffices to prove the lower bound of $q^{b}$. Let $\delta_{0}:=1 \wedge\left(A_{0} / A\right)^{\alpha /(\alpha-\beta)}$. By Chapman-Kolmogorov equation, we only need to consider (5.17) for $t \in\left(0, \delta_{0}\right]$. By (1.20), (1.21) and (3.28), it suffices to prove (5.17) when $|x-y|>3 t^{1 / \alpha}$ and $t \in\left(0, \delta_{0}\right]$. Let $R_{*}$ be the constant defined in Proposition 5.12,

(i) First, we consider the case $R_{*} \geq|x-y|>3 t^{1 / \alpha}$. For every $t \in\left(0, \delta_{0}\right]$, by Proposition 5.10 and Proposition 5.12 with $r=t^{1 / \alpha} / 2$ and the strong Markov property of the process $X^{b}$, we get, by the similar procedure in (5.15), for $R_{*} \geq|x-y|>3 t^{1 / \alpha}$,

$$
\mathbb{P}_{x}\left(X_{2^{-\alpha} \kappa t}^{b} \in B\left(y, t^{1 / \alpha}\right)\right) \geq c_{1} t^{(d+\alpha) / \alpha} \frac{1}{|x-y|^{d+\alpha}} .
$$

Here $c_{1}=c_{1}(d, \alpha, \beta, A)$ is a positive constant. Hence, for $R_{*} \geq|x-y|>3 t^{1 / \alpha}$, by (5.14) and (5.18), we have

$$
\begin{aligned}
q^{b}(t, x, y) & \geq \int_{B\left(y, t^{1 / \alpha}\right)} q^{b}\left(2^{-\alpha} \kappa t, x, z\right) q^{b}\left(\left(1-2^{-\alpha} \kappa\right) t, z, y\right) d z \\
& \geq \inf _{z \in B\left(y, t^{1 / \alpha}\right)} q^{b}\left(\left(1-2^{-\alpha} \kappa\right) t, z, y\right) \mathbb{P}_{x}\left(X_{2^{-\alpha} \kappa t}^{b} \in B\left(y, t^{1 / \alpha}\right)\right) \\
& \geq c_{2} t^{-d / \alpha} t^{(d+\alpha) / \alpha} \frac{1}{|x-y|^{d+\alpha}} \\
& \geq c_{2} \frac{t}{|x-y|^{d+\alpha}}
\end{aligned}
$$

where $c_{2}=c_{2}(d, \alpha, \beta, A)>0$.

(ii) Next, we consider the case $|x-y|>R_{*}>3 t^{1 / \alpha}$. Take $C_{*}=R_{*}^{-1}$. Then $|x-y|>R_{*}=$ $C_{*}^{-1} \geq t / C_{*}$ for $t \in\left(0, \delta_{0}\right]$. The following proof is similar to [8, Theorem 3.6]. For the reader's convenience, we spell out the details here.

Let $R:=|x-y|$ and $c_{+}=R_{*}^{-1} \vee 1$. Let $l \geq 2$ be a positive integer such that $c_{+} R \leq l \leq c_{+} R+1$ and let $x=x_{0}, x_{1}, \cdots, x_{l}=y$ be such that $\left|x_{i}-x_{i-1}\right| \asymp R / l \asymp 1 / c_{+}$for $i=1, \cdots, l-1$. Since $t / l \leq C_{*} R / l \leq C_{*} / c_{+} \leq 1$ and $R / l \leq 1 / c_{+} \leq R_{*}$, we have by (5.14) and (5.19),

$$
q^{b}\left(t / l, x_{i}, x_{i+1}\right) \geq c_{2}\left((t / l)^{-d / \alpha} \wedge \frac{t / l}{(R / l)^{d+\alpha}}\right) \geq c_{2}\left((t / l)^{-d / \alpha} \wedge(t / l)\right) \geq c_{3} t / l .
$$

Let $B_{i}=B\left(x_{i}, R_{*}\right)$, by (5.20),

$$
\begin{aligned}
q^{b}(t, x, y) & \geq \int_{B_{1}} \cdots \int_{B_{l-1}} q^{b}\left(t / l, x, x_{1}\right) \cdots q^{b}\left(t / l, x_{l-1}, y\right) d x_{1} \cdots d x_{l-1} \\
& \geq\left(c_{4} t / l\right)^{l} \geq\left(c_{5} t / R\right)^{c_{+} R+1} \geq c_{6}(t / R)^{c_{7} R} \\
& \geq c_{6}\left(\frac{t}{|x-y|}\right)^{c_{7}|x-y|} .
\end{aligned}
$$


By (5.19), (5.21) and together with the estimates of $\bar{p}_{0}$ in (1.20)-(1.21), we get the desired conclusion.

Proof of Theorem 1.3. Theorem 1.3 now follows from Theorems [5.6, 5.13 and 5.14,

To prove theorem 1.5, we use the main result in [10] of the heat kernel estimates for non-local operators under the non-local Feynman-Kac perturbation. For each Borel function $q(x)$ on $\mathbb{R}^{d}$ and Borel function $F(x, y)$ on $\mathbb{R}^{d} \times \mathbb{R}^{d}$ that vanishes along the diagonal, we define a non-local Feynman-Kac transform for the process $X^{b}$ as follows:

$$
T_{t}^{b, F} f(x)=\mathbb{E}_{x}\left[\exp \left(\int_{0}^{t} q\left(X_{s}^{b}\right) d s+\sum_{s \leq t} F\left(X_{s-}^{b}, X_{s}^{b}\right)\right) f\left(X_{t}^{b}\right)\right] .
$$

Proposition 5.15. Suppose $b$ is a bounded function on $\mathbb{R}^{d} \times \mathbb{R}^{d}$ satisfying (1.2) and (1.19), $q$ is a bounded function on $\mathbb{R}^{d}$ and $|F(x, y)| \leq c\left(|x-y|^{2} \wedge 1\right)$ for some constant $c$. Then for each $f$ in $C_{b}^{2}\left(\mathbb{R}^{d}\right)$,

$$
T_{t}^{b, F} f(x)=f(x)+\int_{0}^{t} T_{s}^{b, F} \mathcal{L}^{b, F} f(x) d s
$$

where

$$
\mathcal{L}^{b, F} f(x)=\mathcal{L}^{b} f(x)+\int_{\mathbb{R}^{d}}\left(e^{F(x, y)}-1\right) f(y) J^{b}(x, y) d y+q(x) f(x) .
$$

Proof. First note that since $X^{b}$ is a semimartingale and $|F(x, y)| \leq c\left(|x-y|^{2} \wedge 1\right)$,

$$
\sum_{s \leq t}\left|F\left(X_{s-}^{b}, X_{s}^{b}\right)\right| \leq c \sum_{s \leq t}\left|X_{s}^{b}-X_{s-}^{b}\right|^{2}=c\left[X^{b}, X^{b}\right]_{t}<\infty
$$

Let $F_{1}=e^{F}-1$ and define

$$
K_{t}=\int_{0}^{t} q\left(X_{s}^{b}\right) d s+\sum_{s \leq t} F_{1}\left(X_{s-}^{b}, X_{s}^{b}\right)
$$

Then by [25, A4.17], the Stieljes exponential

$$
A_{t}:=\operatorname{Exp}(K)_{t}=e^{K_{t}^{c}} \prod_{0<s \leq t}\left(1+K_{s}-K_{s-}\right)=\exp \left(\int_{0}^{t} q\left(X_{s}^{b}\right) d s+\sum_{s \leq t} F\left(X_{s-}^{b}, X_{s}^{b}\right)\right)
$$

is the unique solution to

$$
A_{t}=1+\int_{0}^{t} A_{s-} d K_{s}
$$

For each function $f$ in $C_{b}^{2}\left(\mathbb{R}^{d}\right)$, by Ito's formula, Proposition 5.1 and (5.24), we have

$$
\begin{aligned}
A_{t} f\left(X_{t}^{b}\right)= & f\left(X_{0}^{b}\right)+\int_{0}^{t} f\left(X_{s-}^{b}\right) A_{s-} d K_{s}+\int_{0}^{t} A_{s-} d f\left(X_{s}^{b}\right)+\sum_{s \leq t}\left(A_{s}-A_{s-}\right)\left(f\left(X_{s}^{b}\right)-f\left(X_{s-}^{b}\right)\right) \\
= & f\left(X_{0}^{b}\right)+\int_{0}^{t} A_{s} f\left(X_{s}^{b}\right) q\left(X_{s}^{b}\right) d s+\sum_{s \leq t} f\left(X_{s-}^{b}\right) A_{s-} F_{1}\left(X_{s-}^{b}, X_{s}^{b}\right) \\
& \quad+\int_{0}^{t} A_{s} \mathcal{L}^{b} f\left(X_{s}^{b}\right) d s+\int_{0}^{t} A_{s-} d M_{s}^{f}+\sum_{s \leq t} A_{s-} F_{1}\left(X_{s-}^{b}, X_{s}^{b}\right)\left(f\left(X_{s}^{b}\right)-f\left(X_{s-}^{b}\right)\right) .
\end{aligned}
$$


By taking expectation on both sides and using the Lévy system formula in Proposition 5.3 , we get

$$
\begin{aligned}
T_{t}^{b, F} f(x)= & \mathbb{E}_{x}\left[A_{t} f\left(X_{t}^{b}\right)\right] \\
= & f\left(X_{0}^{b}\right)+\mathbb{E}_{x}\left[\int_{0}^{t} A_{s} f\left(X_{s}^{b}\right) q\left(X_{s}^{b}\right) d s+\int_{0}^{t} A_{s} \mathcal{L}^{b} f\left(X_{s}^{b}\right) d s\right] \\
& +\mathbb{E}_{x}\left[\int_{0}^{t} \int_{\mathbb{R}^{d}} A_{s}\left(f\left(X_{s}^{b}\right)+\left(f(y)-f\left(X_{s}^{b}\right)\right)\right) F_{1}\left(X_{s}^{b}, y\right) J^{b}\left(X_{s}^{b}, y\right) d y d s\right] \\
= & f\left(X_{0}^{b}\right)+\mathbb{E}_{x}\left[\int_{0}^{t} A_{s} \mathcal{L}^{b, F} f\left(X_{s}^{b}\right) d s\right] \\
= & f(x)+\int_{0}^{t} T_{s}^{b, F} \mathcal{L}^{b, F} f(x) d s .
\end{aligned}
$$

That completes the proof.

Proof of Theorem 1.5. Let $b_{0}(x, z)=b(x, z) \mathbb{1}_{|z| \leq 1}(z)$, which is a bounded function on $\mathbb{R}^{d} \times \mathbb{R}^{d}$ satisfying (1.2) and (1.19). By Theorem 1.3, $q^{b_{0}}(t, x, y)$ is continuous on $(0, \infty) \times \mathbb{R}^{d} \times \mathbb{R}^{d}$ and

$$
C_{4} p_{0}(t, x, y) \leq q^{b_{0}}(t, x, y) \leq C_{3} p_{0}(t, x, y)
$$

for all $(t, x, y) \in(0,1] \times \mathbb{R}^{d} \times \mathbb{R}^{d}$. In addition, by Proposition 5.3 and (1.25), for each non-negative function $f$ on $\mathbb{R}^{d} \times \mathbb{R}^{d}$ that vanishes along the diagonal,

$$
\mathbb{E}_{x}\left[\sum_{s \leq T} f\left(X_{s-}^{b_{0}}, X_{s}^{b_{0}}\right)\right]=\mathbb{E}_{x}\left[\int_{0}^{T} \int_{\mathbb{R}^{d}} f\left(X_{s}^{b_{0}}, u\right) J^{b_{0}}\left(X_{s}^{b_{0}}, u\right) d u d s\right] .
$$

and there exist two positive constants $c_{1}$ and $c_{2}$ so that

$$
c_{1}|x-y|^{-(d+\alpha)} \leq J^{b_{0}}(x, y) \leq c_{2}|x-y|^{-(d+\alpha)} .
$$

Set $F(x, y)=\ln \frac{J^{b}(x, y)}{J^{b_{0}}(x, y)}$ and $q(x)=\int_{\mathbb{R}^{d}}\left(J^{b_{0}}(x, y)-J^{b}(x, y)\right) d y$. It is easy to see that $q$ is a bounded function on $\mathbb{R}^{d}$ and $J^{b}(x, y)=J^{b_{0}}(x, y)$ for $|x-y| \leq 1$. Moreover, by the (1.27) and (5.28), there exist two positive constants $c_{3}$ and $c_{4}$ so that $c_{3} \leq \frac{J^{b}(x, y)}{J^{b}(x, y)} \leq c_{4}$ for all $|x-y|>1$ and any bounded $b$ with $\|b\|_{\infty} \leq A$. Hence, there is a positive constant $c_{5}$ so that $|F(x, y)| \leq c_{5}\left(|x-y|^{2} \wedge 1\right)$. Let $T_{t}^{b_{0}, F}$ be the semigroup $T_{t}^{b, F}$ defined by (5.22) but with $b_{0}$ in place of $b$. By (5.26)-(5.28) above and [10, Theorem 1.3], the non-local Feynman-Kac semigroup $\left(T_{t}^{b_{0}, F}, t \geq 0\right)$ has a continuous density $\widetilde{q}(t, x, y)$ and there is a positive constant $c_{6}$ so that for all $(t, x, y) \in(0,1] \times \mathbb{R}^{d} \times \mathbb{R}^{d}$,

$$
c_{6}^{-1} p_{0}(t, x, y) \leq \widetilde{q}(t, x, y) \leq c_{6} p_{0}(t, x, y) .
$$

On the other hand, for each $f$ in $C_{b}^{2}\left(\mathbb{R}^{d}\right)$,

$$
\begin{aligned}
\mathcal{L}^{b_{0}, F} f(x) & =\mathcal{L}^{b_{0}} f(x)+\int_{\mathbb{R}^{d}}\left(e^{F(x, y)}-1\right) f(y) J^{b_{0}}(x, y) d y+q(x) f(x) \\
& =\mathcal{L}^{b_{0}} f(x)+\int_{\mathbb{R}^{d}}\left(J^{b}(x, y)-J^{b_{0}}(x, y)\right)(f(y)-f(x)) d y \\
& =\mathcal{L}^{b} f(x) .
\end{aligned}
$$


By taking $f=1$ in Proposition [5.15, we get $T_{t}^{b_{0}, F} 1=1$. Hence $\widetilde{q}(t, x, y)$ uniquely determines a conservative Feller process $\widetilde{Y}$ with $\left\{T_{t}^{b_{0}, F} ; t \geq 0\right\}$ as its transition semigroup. Proposition 5.15 implies that the distribution of $\widetilde{Y}$ on the canonical Skorokhod space $\mathbb{D}\left([0, \infty), \mathbb{R}^{d}\right)$ is a solution to the martingale problem $\left(\mathcal{L}^{b}, C_{b}^{2}\left(\mathbb{R}^{d}\right)\right)$ and in particular to the martingale problem $\left(\mathcal{L}^{b}, \mathcal{S}\left(\mathbb{R}^{d}\right)\right)$. However by Theorem 1.3, martingale solution to the operator $\left(\mathcal{L}^{b}, \mathcal{S}\left(\mathbb{R}^{d}\right)\right)$ is unique. This yields that $\widetilde{q}=q^{b}$ and so we get the desired conclusion from (5.29).

Acknowledgements. Part of the main results of this paper has been presented at the workshop on "Nonlocal operators: Analysis, Probability and Geometry and Applications", held at ZiF, Bielefeld, Germany from July 9 to July 14, 2012 and at the "Eighth Workshop on Markov Processes and Related Topics" held at Beijing Normal University and Wuyi Shanzhuang from July 16 to July 21, 2012. Helpful comments from the audience, in particular those from Mufa Chen, Mateusz Kwasnicki, and Ting Yang, are gratefully acknowledged.

\section{References}

[1] D. Applebaum, Lévy Processes and Stochastic Calculus. Cambridge University Press, 2004.

[2] M. T. Barlow, R. F. Bass, Z.-Q. Chen and M. Kassmann, Non-local Dirichlet forms and symmetric jump processes. Trans. Amer. Math. Soc. 361 (2009), 1963-1999.

[3] R. F. Bass, Local times for a class of purely discontinuous martingales. Z. Wahrsch. Verw. Gebiete 67 (1984), 433-459.

[4] R. F. Bass and Z.-Q. Chen, System of equations driven by stable processes. Probab. Theory Relat. Fields 134 (2006), 175-214.

[5] R. F. Bass and H. Tang, The martingale problem for a class of stable-like processes. Stochastic Process. Appl. 119 (2009), 1144-1167.

[6] K. Bogdan and T. Jakubowski, Estimates of heat kernel of fractional Laplacian perturbed by gradient operators. Comm. Math. Phys. 271 (2007), 179-198.

[7] Z.-Q. Chen, Symmetric jump processes and their heat kernel estimates. Sci. China Ser. A. 52 (2009), 1423-1445.

[8] Z.-Q. Chen, P. Kim and T. Kumagai, Weighted Poincaré inequality and heat kernel estimates for finite range jump processes. Math. Ann. 342 (2008), 833-883.

[9] Z.-Q. Chen, P. Kim and R. Song, Dirichlet heat kernel estimates for fractional Laplacian under gradient perturbation. Ann. Probab. 40 (2012), 2483-2538.

[10] Z.-Q. Chen, P. Kim and R. Song, Stability of Dirichlet heat kernel estimates for non-local operators under Feynman-Kac perturbation. Trans. Amer. Math. Soc. 367 (2015), 52375270 .

[11] Z.-Q. Chen and T. Kumagai, Heat kernel estimates for stable-like processes on $d$-sets. Stoch. Process Appl., 108 (2003), 27-62.

[12] Z.-Q. Chen and T. Kumagai, Heat kernel estimates for jump processes of mixed types on metric measure spaces. Probab. Theory Relat. Fields, 140 (2008), 270-317. 
[13] Z.-Q. Chen and L. Wang, Uniqueness of stable processes with drift. Proc. Amer. Math. Soc. 144 (2016), 2661-2675.

[14] Z.-Q. Chen and Z. Zhao, Potential theory for elliptic systems. Ann. Probab. 24 (1996), 293-319.

[15] K. L. Chung, Lectures from Markov Processes to Brownian Motion. Springer-Verlag, Berlin and Heidelberg, 1982.

[16] S. N. Ethier and T. G. Kurtz, Markov Processes: Characterization and Convergence. Wiley, New York 1986.

[17] N. Jacob, Pseudo Differential Operators and Markov Processes. I, II and III. Imperial College Press, London, 2001, 2002 and 2005.

[18] V. Kolokoltsov, Symmetric stable laws and stable-like jump diffusions. Proc. London Math. Soc. 80 (2000), 725-768.

[19] T. Komatsu, Markov processes associated with certain integro-differential operators. Osaka J. Math. 10 (1973), 271-303.

[20] T. Komatsu, On the martingale problem for generators of stable processes with perturbations. Osaka J. Math. 21 (1984), 113-132.

[21] P.-A. Meyer, Renaissance, recollements, mélanges, raletissement de processus de Markov. Ann. Inst. Fourier 25 (1975), 464-497.

[22] R. Mikulevicious and G. Pragarauskas, On the martingale problem associated with nondegenerate Lévy operators. Liet. Mat. Journal 32 (1992), 297-311.

[23] A. Negoro,A. and M. Tsuchiya, Stochastic processes and semigroups associate with degenerate Lévy generating operators. Stochastic and Stochastic Reports 26 (1989), 29-61.

[24] R. Schilling and J. Wang: Some theorems on Feller processes: transience, local times and ultracontractivity. Trans. Amer. Math. Soc. 365 (2013), 3255-3286

[25] M. Sharpe. General theory of Markov processes. Academic Press, Boston, 1988.

[26] D. Stroock, Diffusion processes associated with Lévy generators. Z. Wahrsch. Verw. Gebiete 32 (1975), 209-244.

[27] H. Tanaka, M. Tsuchiya and S. Watanabe, Perturbation of drift-type for Lévy processes. J. Math. Kyoto Univ. 14 (1974), 73-92.

[28] M. Tsuchiya, On some perturbation of stable processes. Proc. 2nd Japan-USSR Symposium on Probab. Theory. Lect. Notes Math. 330 (1973), 490-497.

[29] C. Wang. On estimates of the density of Feynman-Kac semigroups of $\alpha$-stable-like processes. J. Math. Anal. Appl. 348 (2008), 938-970.

[30] J. -M. Wang, Laplacian perturbed by non-local operators. Math. Z. 279 (2015), 521-556. 


\section{Zhen-Qing Chen}

Department of Mathematics, University of Washington, Seattle, WA 98195, USA

E-mail: zqchen@uw.edu

\section{Jie-Ming Wang}

School of Mathematics and Statistics, Beijing Institute of Technology, Beijing 100081, P. R. China.

E-mail: wangjm@bit.edu.cn 